\title{
A DIGITAL MICROFLUIDIC PLATFORM FOR HUMAN PLASMA PROTEIN DEPLETION
}

\author{
by \\ NINGSI MEI \\ A thesis submitted to the Department of Chemistry in conformity with the \\ requirements for the Degree of Master of Science
}

Queen's University

Kingston, Ontario, Canada

May, 2014

Copyright (C Ningsi Mei, 2014 


\section{ABSTRACT}

Digital microfluidics (DMF) is an emerging liquid-handling technique that facilitates manipulation of discrete droplets across an array of electrodes. Although the working principle of droplet movement is still under debate, it has gained significant interest as the technique has been applied to various applications in biology, chemistry and medicine. With recent advances in rapid prototyping and multilayer fabrication techniques using printed circuit boards, DMF has become an attractive and alternative solution to conventional macroscale fluidics techniques with additional capability of sample size reduction, faster analysis time, full automation, and multiplexing.

In this thesis, we explore the use of DMF for human plasma protein depletion due to its multiple advantages. The high abundance of human serum albumin (HSA) and immunoglobulins (Igs), which constitute $80 \%$ of total plasma proteins, is a major challenge in proteome studies. Unfortunately, conventional methods to deplete high abundant proteins (e.g. macro LC-columns) are labourintensive, require dilution of sample, and run the risk of sample loss. Furthermore, most techniques lack the ability to process multiple samples simultaneously. Hence, we developed a new method of protein depletion using anti-HSA and Protein A/G 
immobilized paramagnetic beads manipulated by DMF to deplete HSA and IgG from human plasma.

Toward this goal, prototype DMF devices and electronic controller were designed, built and characterized (Chapter 2). Preliminary depletion experiments were first optimized in-tubes and then adapted for DMF manually (Chapter 3). At last, the entire depletion process was performed on DMF using an automated controller system (Chapter 4). Results showed that the protein depletion efficiency for immunoglobulin $\mathrm{G}$ ( $\mathrm{IgG}$ ) and HSA in 10 minutes for four samples simultaneously was as high as $98 \%$, and an approximately 3 -fold increase in signalto-noise ratio after depletion was demonstrated by MALDI-MS analysis. The depletion process is sufficient for a tryptic digest to be performed on a model protein, cytochrome $\mathrm{C}$, where $89 \%$ sequence coverage was obtained for a depleted sample. Although some improvements such as on-chip sample processing (e.g. digestion) need to be carried out as future work, we anticipate that the new technique is a significant alternative for applications involving protein depletion steps. 


\section{CO-AUTHORSHIP}

All research undertaken for this thesis was completed under the supervision of Dr.

Richard D. Oleschuk and Dr. Aaron A. Wheeler. Part of the work presented in Chapters 3 and 4 was co-authored by Brendon Seale and Alphonsus Ng (Ph. D. students of Dr. Wheeler) and Dr. Wheeler. 


\section{ACKNOWLEDGMENTS}

I would like to give my deepest gratitude to my supervisor, Dr. Richard D. Oleschuk, for the guidance, inspiration and encouragement during my entire master's project research. As the first person in our group who has worked on DMF, I often felt struggling and frustrating throughout the entire project. Many thanks to Dr. Richard Oleschuk for his kind help, encouragement and recognition on my work ability; he has been the best supervisor I have ever had. I feel very blessed to be part of our comfortable and aspirant "O" group, and I enjoyed working and being with all my group members.

I am very grateful to Dr. Aaron Wheeler and his students, Brendon Seale and Alphonsus Ng and Victor Lee. Dr. Wheeler gave me the opportunity to work in his group at University of Toronto on the exciting DMF project and his students, in particular Brendon Seale and Alphonsus $\mathrm{Ng}$, have been very helpful and showed me around as mentors, and contributed greatly on my experimental work. Without their generosity of acceptance and valuable guidance throughout my project using antibody immobilized magnetic beads for human plasma protein depletion, I would not have accomplished my research project. I am also very thankful to Dr. Wheeler's former student, Dr. Hao Yang, for his help and patience with my questions and generously providing me with valuable ideas on the project. 
I would like to acknowledge my supervisory committee members, Dr. Stephen Brown and Dr. Jean-Michel Nunzi, for their valuable suggestions. Particular thanks to David McLeod and Dr. Jiaxi Wang for their critical help on the sample analysis using mass spectrometry.

Likewise, I would like to thank Dr. Henry Lee and Yimin Zhou for their help and training on the fabrication of chips in the ECTI clean room at the University of Toronto.

Special thanks to my beloved parents for their endless love, support, and encouragement, in particular to my mother. Without her generous help, support, and belief in my ability, I could not have accomplished this master's project.

Finally, I would like to thank Queen's University and the Natural Sciences and Engineering Research Council for financial support of this project, Dr. Mingxian Huang from Innosep Company Limited for his kind donation of magnetic beads, and CMC Microsystems for their help on microfluidic chip design/fabrication, and providing training opportunities. 


\section{TABLE OF CONTENTS}

ABSTRACT

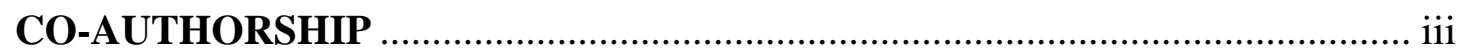

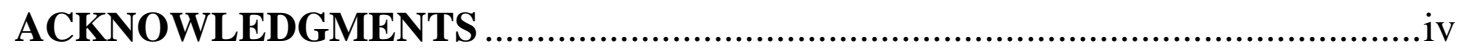

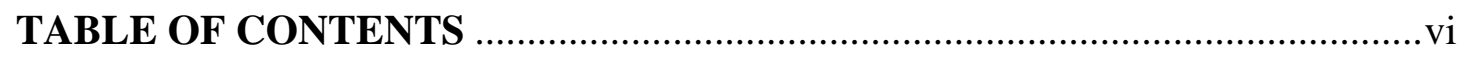

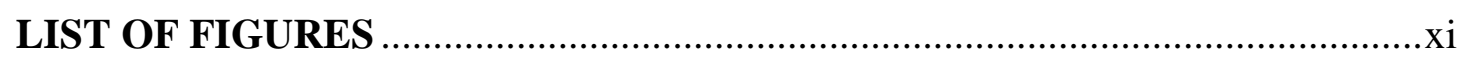

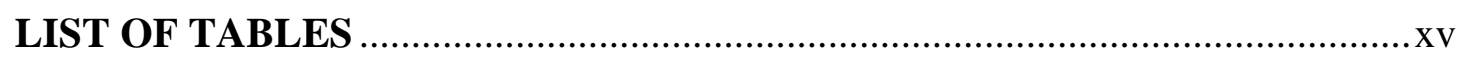

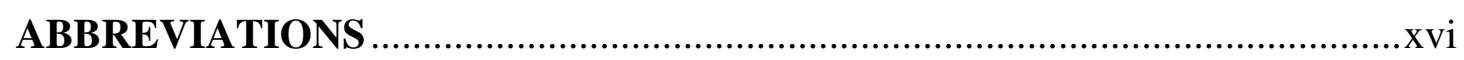

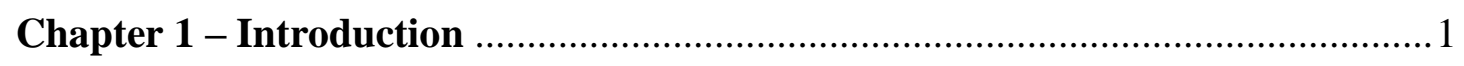

1.1 Overview of Digital Microfluidics (DMF) .............................................. 1

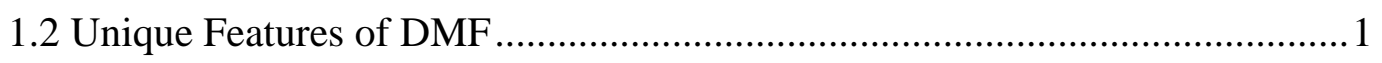

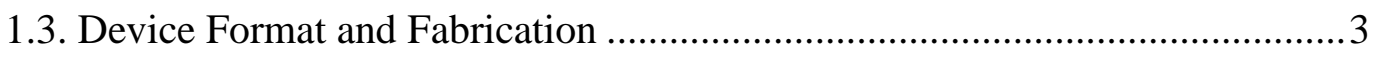

1.3.1 Device Formats of DMF .............................................................

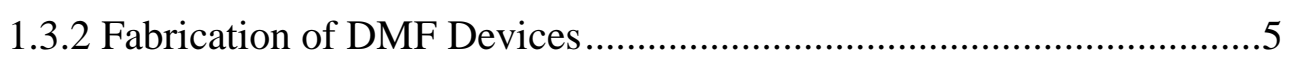

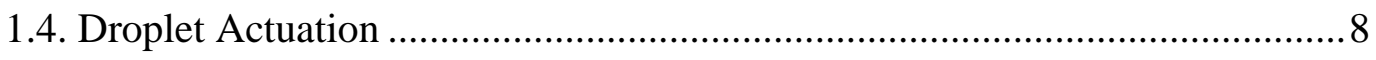


1.6.3 Proteomics 18

1.6.4 Cell-Based Assays 19

1.6.5 Immunoassays .20

1.6.6 Medicinal and Clinical Diagnostics 21 
Chapter 3 - Application of Protein Depletion Off-Chip and On-Chip Using 
3.2 Results and Discussion 49

Chapter 4 - Automated Protein Depletion On-Chip 55

4.1 Experimental 55

4.1.1 Reagents and Materials .55

4.1.2 On-Chip Protein Depletion Reagents..... .55

4.1.3 Off-Chip MALDI and LC-MS/MS Protein Depletion Analysis Reagents .56

4.1.4 Device Fabrication and Operation .57

4.1.5 On-Chip Protein Depletion Protocol .61

4.1.6 Fluorescent Characterization of On-Chip Depletion. .62

4.1.7 MALDI-MS Characterization of On-Chip Depletion .64

4.1.8 Proteomic In-Solution Digestion and Identification of Peptides with MALDI-MS and LC-MS/MS .65

4.2. Results and Discussion 67

4.2.1 DMF Device and Method .67 
4.2.4 Proteomic In-Solution Digestion and Identification of Peptides with

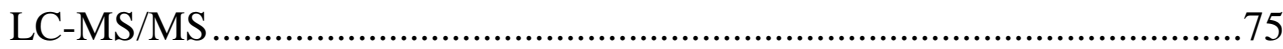

Chapter 5 - Conclusions and Future Work 79

5.1 Conclusions

5.2 Future Work 81 


\section{LIST OF FIGURES}

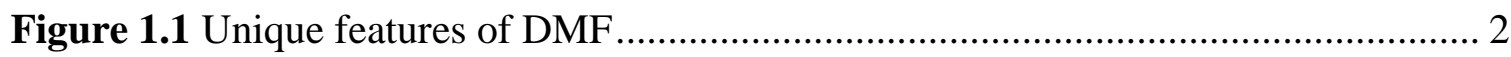

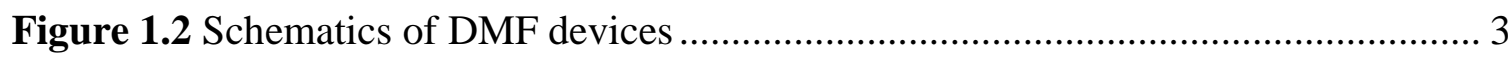

Figure 1.3 Picture of a flexible "All-Terrain Droplet Actuation" device moving a droplet from a warm to a cool area.......................................................... 4

Figure 1.4 Schematic diagram of key components for fabrication of a DMF device....... 7

Figure 1.5 Distribution of electrodynamic forces as a function of electric field intensity.

Figure 1.6 Diagram of effect of dipole polarization on wetting. 12

Figure 1.7 Applications of DMF 17

Figure 2.1 Electrowetting experimental setup. 27

Figure 2.2 a) DMF chip using a PCB platform design is shown with all dimensions in $\mu \mathrm{m}$; b) Parylene-C vapor deposition coating system; c) Teflon®-AF spin coating system 30

Figure 2.3 Glass chip design 30 
Figure 2.4 CMC Microsystems (Microsystem Integration Platform, MIP) system controller for microfluidic devices. 33

Figure 2.5 DCPower Soft Front Panel - software which controls the NI PXI-4130 ...... 34

Figure 2.6 AC Function Generator Soft Front Panel - software which controls the NI PXI-5422 34

Figure 2.7 Manipulation of a droplet on a two-plate DMF device fabricated in the Wheeler group. 35

Figure 2.8 Screen shots from video clips of electrowetting. 37

Figure 2.9 Captured images from video clips of droplet movement on PCB chip without ITO plate 38

Figure 2.10 Images captured from video clips of droplet movement on glass chip without the ITO top plate (i.e. single plate) 39

Figure 2.11 Screen captures from video of droplet movement on a glass chip 40

Figure 2.12 Non-functional glass DMF chip coated with Parylene-C with white scratch traces observed 41 
Figure 3.1 Schematics for magnetic bead-based separation of low-abundant proteins on a DMF platform.

Figure 3.2 Images captured from a movie, which depicts the eight steps involved with the manual magnetic bead-based protein depletion process. .48

Figure 3.3 Magnet-actuated droplet manipulation .49

Figure 3.4 MALDI-MS Spectra for high-abundance and low-abundance proteins (IgG and transferrin) . .51

Figure 3.5 MALDI-MS Spectra for high-abundance and low-abundance proteins (IgG and transferring .52

Figure 3.6 Magnetic actuated droplet containing paramagnetic particles moving back and forth .53

Figure 3.7 Separation of water droplet from magnetic particles .54

Figure 4.1 Device and Processing scheme. 58

Figure 4.2 Integrated platform for DMF particle-based immunoassays. 59

Figure 4.3 Screenshot from the custom Microdrop software demonstrating live video overlay. 59 
Figure 4.4 Frames from a video depicting the process of protein depletion from a sample. The dark areas on the array are the magnetic beads. 62

Figure 4.5 On-chip depletion kinetics and efficacy. 69

Figure 4.6 MALDI Spectra of sample comprising HSA $(0.5 \mathrm{mg} / \mathrm{mL}), \operatorname{IgG}(2 \mathrm{mg} / \mathrm{mL})$ and Hemopexin $(0.1 \mathrm{mg} / \mathrm{mL})$ 74

Figure 4.7 MALDI-MS spectrum for digestion of cytochrome C, 11 peptides matched, score 225 and sequence coverage of $68 \%$. 76 


\section{LIST OF TABLES}

Table 1.1 Capabilities of and challenges for DMF............................................... 2

Table 4.1 Comparison of S/N ratios for Ion Intensities in MALDI-MS spectra for control, following a single, and double depletion, with the DMF/magnetic

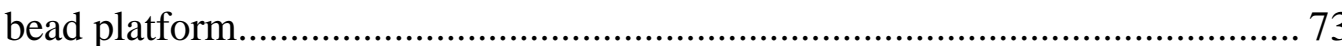

Table 4.2 Sequence Peptides of Trypsin Digested Cytochrome C using LCMS/MS 


\section{ABBREVIATIONS}

AA

$\mathrm{AC}$

$\mathrm{ACN}$

AP

ATDA

ATP

BSA

CAD

$\alpha-\mathrm{CHCA}$

$\mathrm{CP}$

DBS

DC

DI $\mathrm{H}_{2} \mathrm{O}$

DMF

ECTI

ESI

EWOD

FDP

FITC

HAPs

HSA

$\mathrm{Hz}$

$\mathrm{IgG}$

Igs

ITO

$\mathrm{kHz}$

LC-MS/MS

LED

LOC

MALDI

$\mu \mathrm{m}$

$\mathrm{mm}$

MS

$\mathrm{N}_{\mathrm{RMS}}$

$\mathrm{nm}$
Amino acids

Alternating current

Acetonitrile

Alkaline phosphatase

All Terrain Droplet Actuation

Adenosine triphosphate

Bovine serum albumin

Computer-aided design

$\alpha$-cyano-4-hydroxycinnamic acid

Cyclic peptide

Dried blood spot

Direct current

Deionized water

Digital microfluidics

Emerging Communications Technology Institute

Electrospray ionization

Electrowetting-on-dielectric

Fluorescein diphosphate

Fluorescein isothiocyanate

Highly abundant proteins

Human serum albumin

Hertz

Immunoglobulin $\mathrm{G}$

Immunoglobulins

Indium-tin oxide

Kilohertz

Liquid chromatography tandem-mass spectrometry

Light-emitting diode

Lab-on-a-chip

Matrix assisted laser desorption ionization

Micrometers

Millimeter

Mass spectrometry

Root-mean square noise

Nanometer 
PCB

PMT

SA

$\mathrm{S} / \mathrm{N}$

tBuNC

TFA

TNFC
Printed circuit board

Photomultiplier

Sinapinic acid

Signal-to-noise

tert-butyl isocyanide

Trifluoroacetic acid

Toronto Nanofabrication Centre 


\section{Chapter 1 Introduction}

\subsection{Overview of Digital Microfluidics (DMF)}

Digital microfluidics (DMF) has recently emerged as a liquid-handling technology for manipulating discrete droplets ranging from picoliters up to milliliters in volume by applying a series of electrical potentials to an array of electrodes. ${ }^{1}$ Like the more established microchannel techniques, DMF has been used to miniaturize a wide range of lab-on-a-chip (LOC) applications, with the advantages of reduced reagent/sample consumption, fast reaction kinetics, and capacity to integrate multiple components. $^{2,3}$ However, DMF possesses several unique features in contrast to microchannels making it an attractive fluid manipulation platform. ${ }^{1,4,5}$

\subsection{Unique Features of DMF}

DMF is capable of addressing each reagent droplet individually rather than in series, which allows for droplets to be moved independently from each other (Figure 1.1a). ${ }^{6}$ A second feature is reagent isolation where DMF can manage liquid/solid samples with no risk of clogging (Figure 1.1b). ${ }^{7}$ Furthermore, DMF geometry is compatible with a large range of droplet volumes, making it a good match for array-based biochemical applications (Figure 1.1c). ${ }^{8}$ These key capabilities and challenges for DMF are summarized in Table 1.1. ${ }^{1}$ 
Herein, a summary of DMF including device formats, fabrication methods, theory of droplet actuation, integration, and a variety of applications in biology, chemistry and medicine, are discussed.

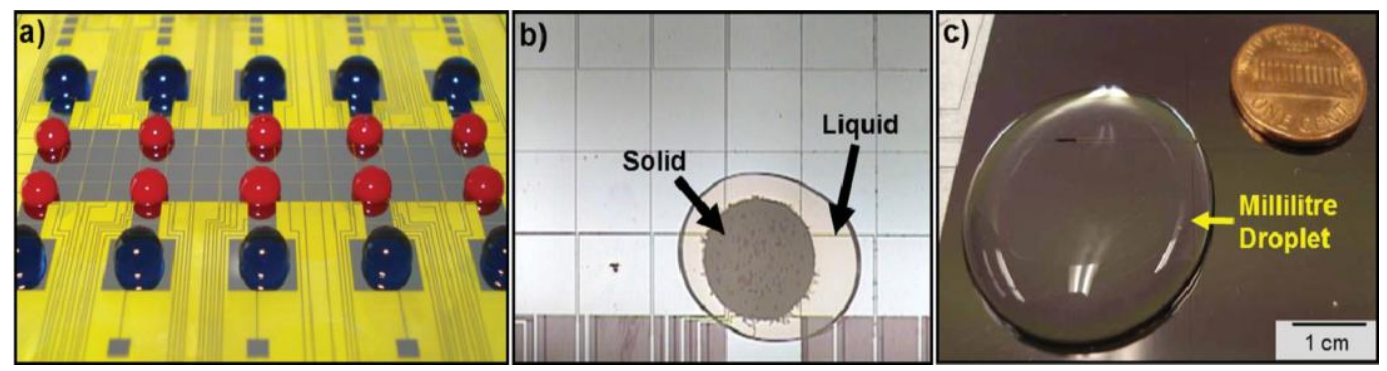

Figure 1.1 Unique features of DMF a) DMF platform controlling twenty reagent droplets with no need for external hardware (i.e. connectors, valves, and pumps). Reproduced with permission from ref. 9. Copyright 2011 Futurity. org. b) Picture of extraction liquid processing a solid dried blood spot (DBS) by DMF. Reproduced with permission from ref. 10. Copyright 2011 The Royal Society of Chemistry. c) Picture of a DMF platform used to manipulate a $\sim 3 \mathrm{~mL}$ sample droplet. Reproduced with permission from ref. 11 . Copyright 2008 The Royal Society of Chemistry.

Table 1.1 Capabilities of and challenges for DMF. Reproduced from ref. 1.

\section{Capabilities Challenges}

\begin{tabular}{ll}
\hline - Easy to manipulate reagent droplets & - Not suitable for chemical separations \\
with no need for pumps, tubing and & or continuous-flow synthesis \\
microvalves & \\
- Can handle wide range of volumes & - Incompatible with high temperatures \\
(nL-mL), suitable for preparative & and pressures
\end{tabular}
applications

- Compatible with aqueous and organic solvents

- Difficulty moving concentrated biological samples without additives or oil matrix

- Straightforward control over different

- Incompatible with centrifugation phases

-Dielectric breakdown with high voltage usage 


\subsection{Device Format and Fabrication}

\subsubsection{Device Formats of DMF}

There are two common configurations of DMF devices, ${ }^{12}$ two-plate (closed) (Figure1.2 a) and single-plate (open) (Figure 1.2 b) devices. In the closed format, droplets are sandwiched between a top ground plate, typically formed from unpatterned, transparent indium tin oxide (ITO) coated glass, and a bottom plate containing patterned actuation electrodes produced using photolithography. In the open format, droplets are positioned on a single plate, which houses both ground and actuation electrodes in a sideby-side geometry. In both geometries, droplets are isolated from the electrodes by an insulating, dielectric coating; all surfaces are further covered by a hydrophobic layer to facilitate smooth droplet movement.

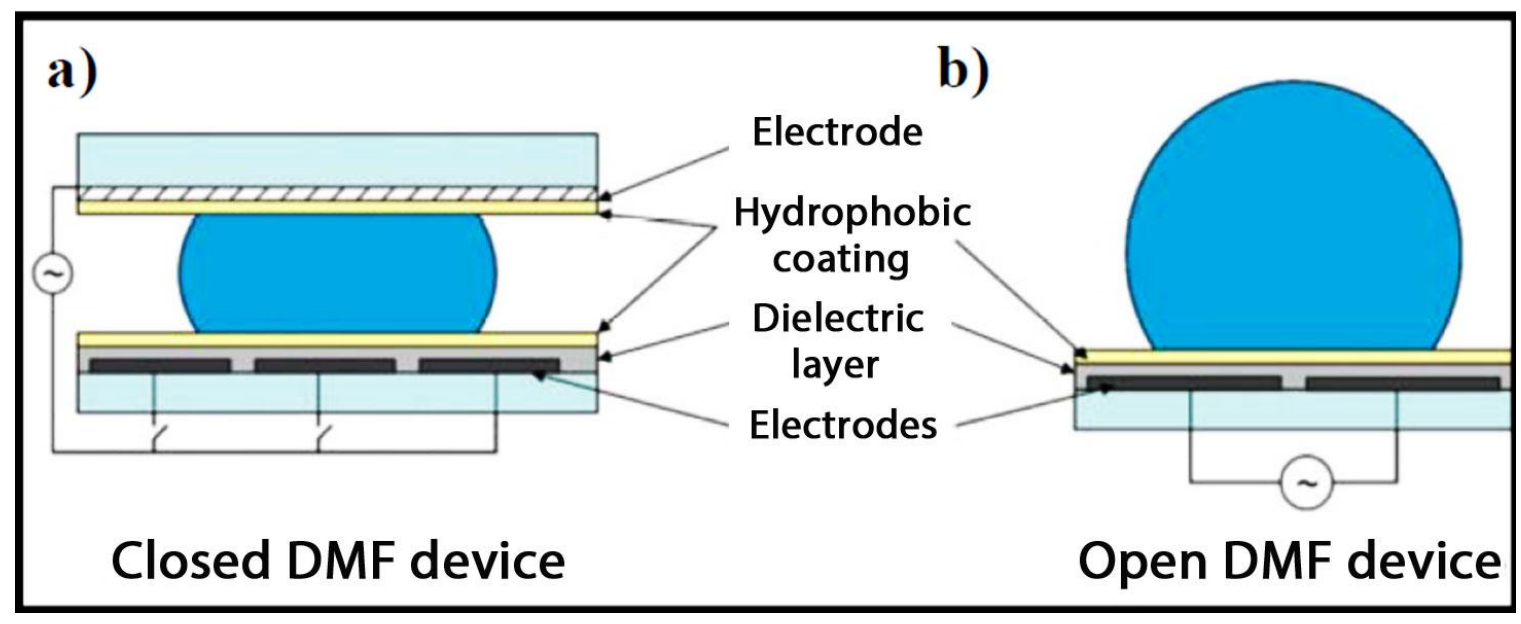

Figure 1.2 Schematics of DMF devices a) Closed DMF device b) Open DMF device. Reproduced with permission from ref. 12. Copyright 2009 Advanced Materials. 
The open and closed DMF configurations have complementary advantages. The open format facilitates faster sample and reagent mixing, ${ }^{13}$ the capacity to move large droplets, ${ }^{11}$ and better access to samples for external detectors or pipette-based liquid handling; ${ }^{14}$ however, it suffers from increased sample evaporation and inability to perform droplet dispensing and splitting. ${ }^{14,15}$ In contrast, closed DMF devices are best suited for a wide range of droplet operations (i.e. dispensing, moving, splitting, and merging). ${ }^{16}$ Therefore, most DMF based biochemical/clinical applications have so far been done on closed devices although open devices may be advantageous for preparative applications in which analytes are recovered after droplet actuation. ${ }^{12}$

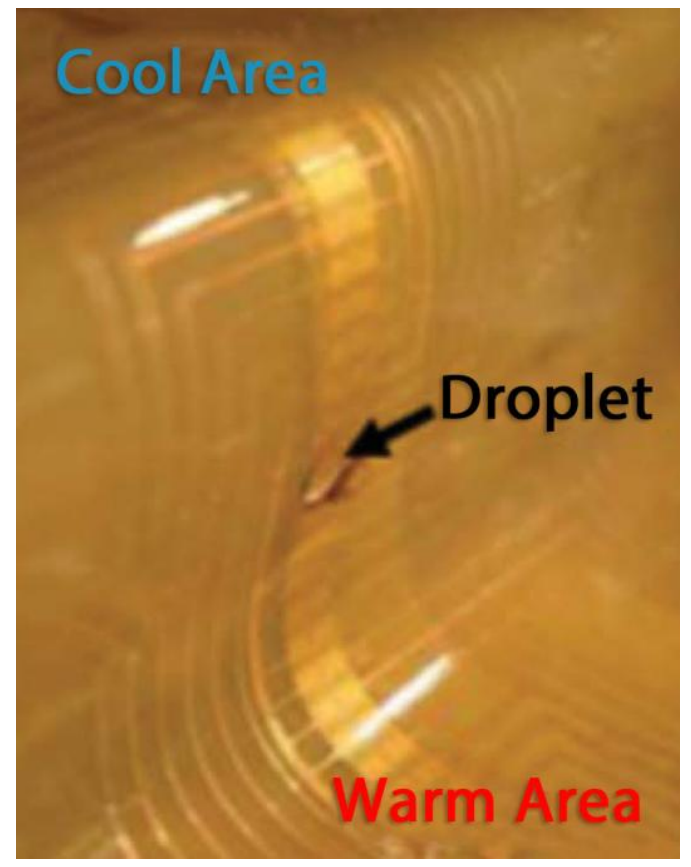

Figure 1.3 Picture of a flexible "All-Terrain Droplet Actuation" device moving a droplet from a warm to a cool area. Reproduced with permission from ref. 11. Copyright 2008 The Royal Society of Chemistry. 
In addition to the closed and open DMF formats, flexible devices have recently become a third format for DMF, as it facilitates droplet actuation on non-planar surfaces, permitting the integration of multiple physicochemical environments on the same device. ${ }^{1}$ For instance, Abdelgawad et al. ${ }^{11}$ described the format of "All Terrain Droplet Actuation (ATDA)" using devices fabricated on copper sheets (Figure 1.3), which were capable of droplet actuation on inclined, declined and inverted surfaces, and useful for applications requiring temperature cycling.

Another key distinction in DMF format is the nature of the matrix surrounding droplets on the device. While many DMF devices are used to actuate droplets in air, another common technique uses droplets suspended in oil, ${ }^{7,17}$ which prevents evaporation, reduces the surface fouling and the surface energy. Thus, this technique allows for lower electrical potentials for droplet actuation. However, oil-immersed systems have several disadvantages, including the requirement of gaskets or other structures to contain the oil bath, the potential for liquid-liquid extraction of analytes into the surrounding oil, ${ }^{11}$ the incompatibility with oil-miscible liquids (e.g. organic solvents), and the incompatibility with assays requiring the droplets to dry onto the device surface. ${ }^{18}$

\subsubsection{Fabrication of DMF Devices}

DMF devices are typically fabricated using metal deposition, standard photolithography processes, wet etching, deposition or thermal growth of a dielectric layer, and deposition of a hydrophobic coating ${ }^{19}$ in a clean room facility. As the fabrication process influences the allowable geometries, the proper choice of device 
substrate becomes important. ${ }^{20}$ Glass and silicon have been widely used as substrates because of their accessibility and compatibility with standard clean room protocols. The main drawbacks of using glass and silicon are cost and wire routing since it is impractical to fabricate glass with multilayer techniques. ${ }^{21}$ Thus, there is a current trend toward the use of printed circuit board (PCB) substrates due to their low cost and compatibility with batch fabrication. ${ }^{13}$ In addition, PCB substrates also allow for isolation of contact wires from driving electrodes by means of vertical interconnects between multiple conducting layers. $^{18}$

As shown in Figure 1.4, each device is fabricated from a bottom plate with individually addressable electrodes and a top plate fabricated as a single, large electrode. For the glass substrate, electrodes in the bottom plate are usually formed from chromium and gold; whereas for the PCB substrate, electrodes are commonly formed from copper. The insulating dielectric layer can be formed using a variety of techniques, including vapor deposition (parylene), thermal growth (silicon oxide), or spin-coating (PDMS or SU-8). ${ }^{22}$ 


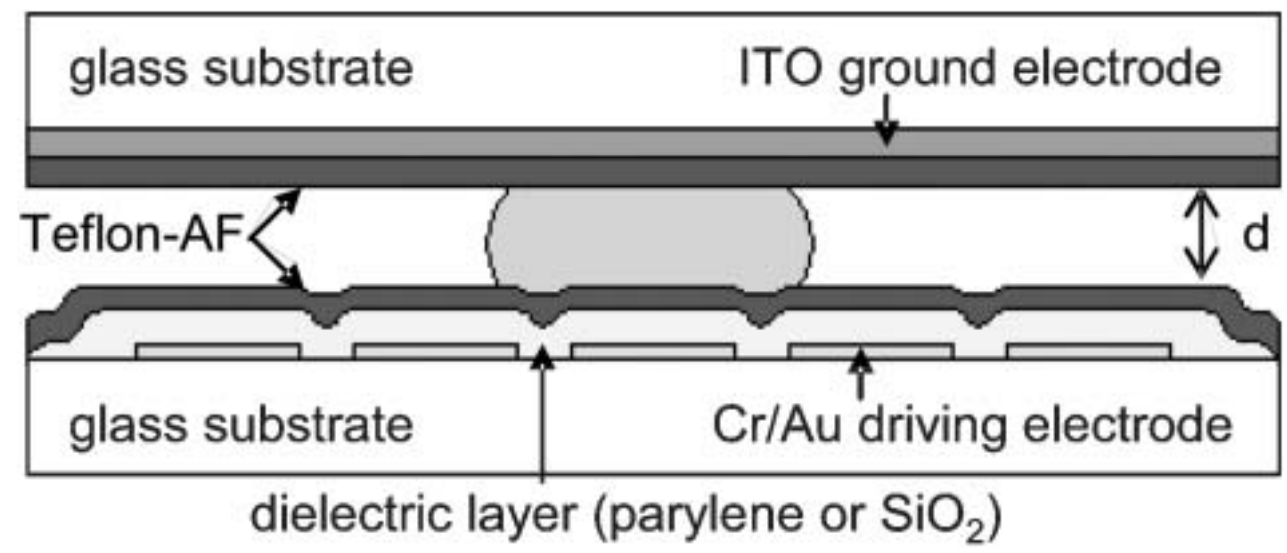

Figure 1.4 Schematic diagram of key components for fabrication of a DMF device. The bottom plate has an array of conductive electrodes, dielectric layer (parylene or $\mathrm{SiO}_{2}$ ) and hydrophobic Teflon ${ }^{\circledR}$-AF coating. The top plate has a single ITO electrode and Teflon ${ }^{\circledR}$ AF coating. The droplet is sandwiched between the two plates with spacing between plates, $d$. Reproduced with permission from ref. 23. Copyright 2006 The Royal Society of Chemistry.

Although parylene-C has been the most used dielectric layer, Abdelgawad et al. ${ }^{24}$ found that PDMS-coated devices had flatter surfaces, which rendered droplet motion more facile and smooth, and were capable of moving droplets across inter-electrode gaps (non-interdigitated) as large as $150 \mu \mathrm{m}$. More recently, Dhindsa et al. $^{25}$ also discovered that parylene HT, the newest commercially available variant of Parylene that replaces the alpha hydrogen atom of the $\mathrm{N}$ dimer with fluorines, exhibits improved resistance to dielectric failure as compared to parylene-C, and it allows operation at low voltage (15V), which demonstrates that Parylene HT is a promising candidate for low-voltage and largearea electrowetting devices. The thickness of the deposited dielectric layer on the substrate is critical to high device yield. Dielectric layers that are too thin lead to nonuniform or incomplete coverage of the electrode array resulting in dielectric breakdown, and electrolysis. ${ }^{26}$ Alternatively, excessively thick dielectric layers require very high 
actuation voltages. Typically, the thickness of the dielectric layer, ranges from 3-14 $\mu \mathrm{m}$, needs to be slightly thicker than the metal substrate thickness, and it can be measured using an optical profilometer. Finally, the hydrophobic coating is usually formed by spincoating a thin layer of Teflon ${ }^{\circledR}$-AF followed by post-baking on a hot-plate, and is coated on both the ITO glass slide (top plate) and electrode patterned layer (bottom plate).

As many academic and industrial groups do not have access to clean room facilities, efforts have been made to fabricate DMF devices from inexpensive, accessible methods such as micro-contact printing ${ }^{27}$, laser printing, ${ }^{24}$ and rapid marker masking techniques. ${ }^{28}$ These techniques have made DMF into a technology that is accessible and useful for a number of applications.

\subsection{Droplet Actuation}

\subsubsection{Physics of Droplet Actuation}

There is still considerable debate about the mechanism(s) of droplet actuation in DMF. However, the most widely accepted justification for moving droplets was described by the Fair ${ }^{7}$ and $\mathrm{Kim}^{8}$ groups, and was termed "electrowetting-on-dielectric" (EWOD) in the early 2000s. Droplet movement on a DMF device is achieved by applying a sequence of alternating current (AC) / direct current (DC) voltages. The driving force acting on the droplet is initially proportional to that applied voltage but saturates beyond a certain threshold. Forces that affect droplet movement can be divided into driving and resistive forces. ${ }^{29}$ The driving force, F, can be derived from the Young-Lippman equation ${ }^{30}$ : 


$$
\mathrm{L} \gamma\left(\cos \theta-\cos \theta_{0}\right)=\frac{\varepsilon_{0} \varepsilon_{\mathrm{r}} L V^{2}}{2 \mathrm{~d}}
$$

where: $L$ is the length of the contact line overlapping the actuated electrode, $\theta$ and $\theta_{0}$ are the static contact angles with and without applied voltage, respectively; $\varepsilon_{\mathrm{r}}$ is the relative permittivity of the dielectric; $\varepsilon_{0}$ is the permittivity of free space; $\mathrm{V}$ is the applied voltage; $\gamma$ is the liquid/filler media surface tension; and $d$ is the dielectric thickness.

The resistive forces are viscous-drag and friction forces, which depend upon the viscosity and the surface tension of the droplet. The EWOD convention arose from the fact that the contact angle between an aqueous droplet and the device surface is reduced (i.e. wetted) during droplet movement. ${ }^{12}$ In this scheme, the two phenomena (droplet wetting and movement) are viewed as being cause-and-effect: droplet movement was understood as being a consequence of capillary pressure arising from non-symmetrical contact angles across the droplet. ${ }^{12}$

However, this justification fails to explain droplet motion for dielectric (non-ionic) liquids such as organic solvents or for low-surface-tension liquids with no apparent changes in contact angles; ${ }^{23,31}$ nor can it explain related phenomena such as contact angle saturation (i.e. the observed limit on contact angle change above a threshold in applied potential). Therefore, a more direct and generalized understanding of the physics of droplet actuation was derived from an electromechanical model, which uses an electronic circuit to represent a droplet sandwiched in a DMF device (i.e. capacitance and resistance for droplet and dielectric layers). ${ }^{32,33}$ In this electromechanical framework, it explains 
both wetting and droplet movement phenomena in terms of the electrical forces generated on free charges in the droplet meniscus (in case of conductive liquids) or on dipoles inside of the droplet (in case of dielectric liquids). As shown in Figure 1.5, when an electrical potential is applied to the electrode where the droplet is sitting adjacent to it, opposite charges accumulate on either side of the dielectric. ${ }^{20,34}$ As a result, the electrodynamic forces, which are most dense on the front of the droplet near the threephase contact line, serve as "electrostatic handles" to pull the droplet over the activated electrode. These electrodynamic forces can be derived from the capacitive energy stored in the system, which is a function of frequency and the droplet position along the moving axis in the direction of droplet propagation. Differentiating this energy with respect to the position along the moving axis yields the driving force. A complete derivation with supporting mathematical equations can be found in a previous report by the Garrell group. ${ }^{23}$ Using this model, any device geometry and liquid composition can be accessed, and forces can be evaluated and optimized for best performance. 


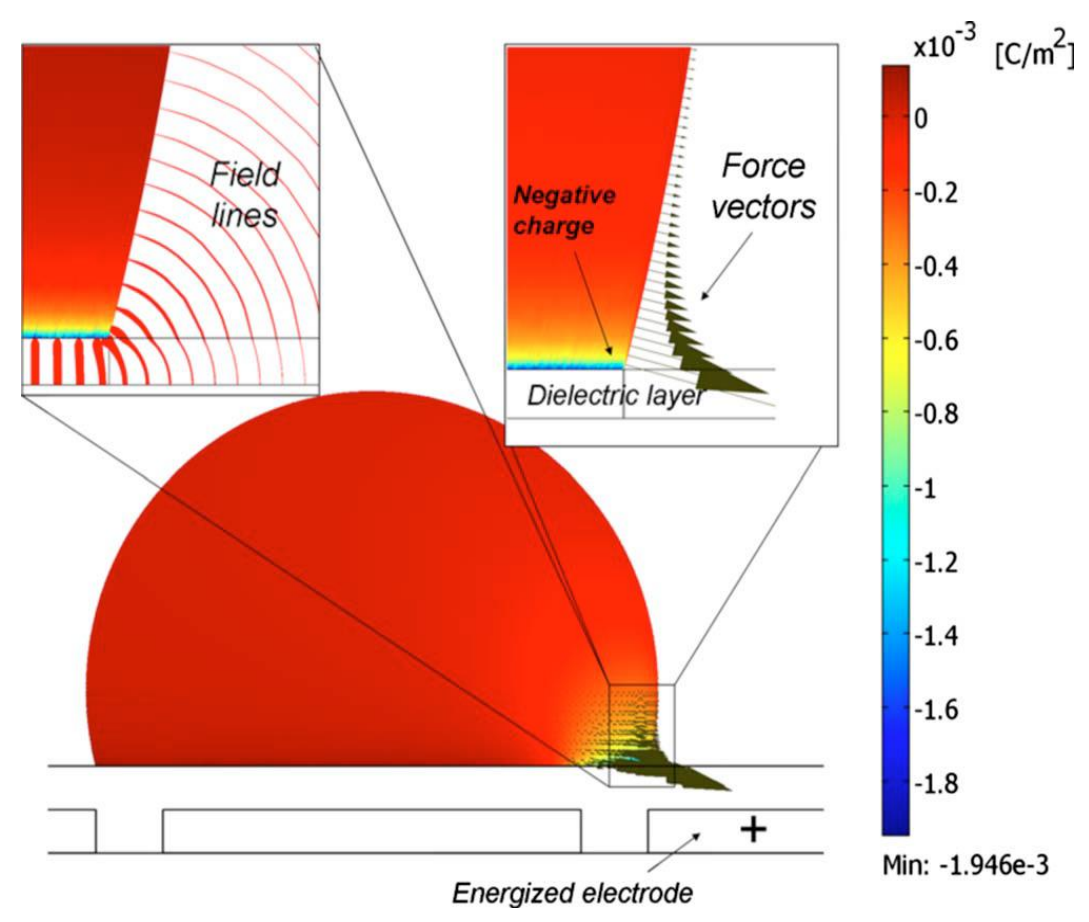

Figure 1.5 Distribution of electrodynamic forces as a function of electric field intensity. Black arrows are most dense on the front of the droplet near the three-phase contact line because of negative charge accumulation in this region. The left inset shows electric field lines in the vicinity of the three-phase contact line (line thickness scales with field intensity), whereas the right inset shows the force distribution in the same region (in the vertical plane passing through droplet center). Reproduced with permission from ref. 34 . Copyright 2009 American Institute of Physics.

\subsubsection{Use of AC vs. DC Actuation Voltages}

One of the most observable sources of division between different microfluidic labs and companies rests upon whether AC or DC voltages should be applied to induce actuation movement. Nagiel ${ }^{35}$ under the supervision of Dr. Richard Fair investigated this question by characterizing the effect of frequency on threshold voltage for droplet actuation. Furthermore, they examined the capacitive changes with respect to an applied DC or AC voltage. Threshold voltage in the context of DMF is defined as the minimum voltage required inducing droplet movement. It was shown that high $\mathrm{AC}$ frequencies 
resulted in higher actuation threshold to the point where DC was preferable. However, lower AC frequencies (e.g. $10 \mathrm{~Hz}$ ) produced minimal threshold voltages.

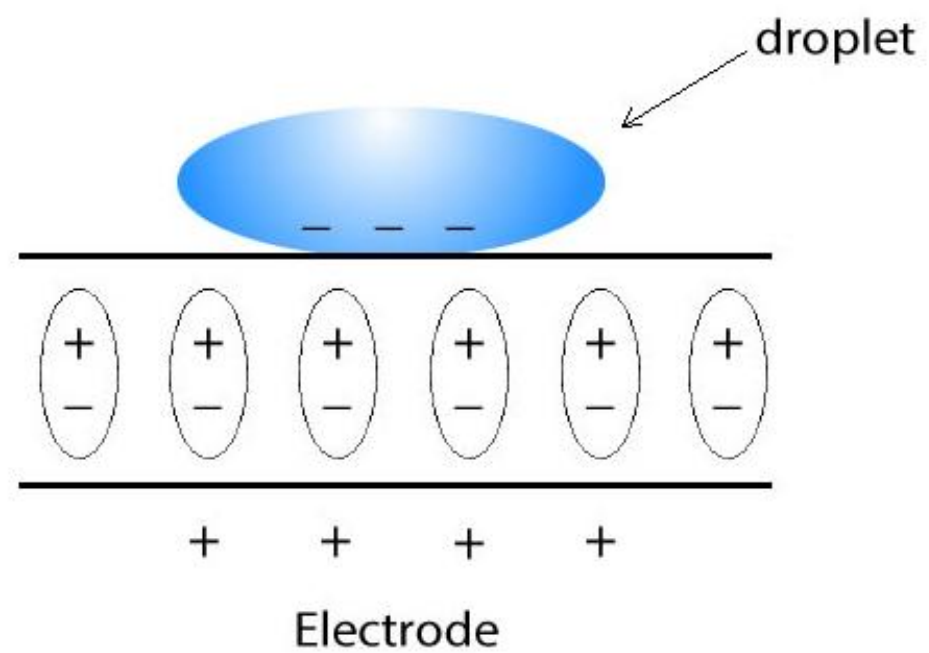

Figure 1.6 Diagram of effect of dipole polarization on wetting. Positive charges accumulated at the electrode surface and all the negative charges in droplet move toward the hydrophobic surface due to electrostatic interaction. The remnant polarization within the dielectric layer inducing increased wetting of the droplet. Reproduced from ref. 35.

In terms of capacitive effects, there is a hysteresis that occurs whenever DC voltages are utilized for droplet movement. This is most likely due to a persistent polarization within the dielectric layer which promotes surface wetting. As shown in Figure 1.6, positive charges accumulate at the electrode surface and negative charges within droplet migrate to the surface due to electrostatic attraction. This causes all negative ions in solution to be deposited onto the hydrophobic surface, which would also promote surface fouling of charged molecules such as proteins. 
However, the hysteresis does not occur when AC is used. Although low frequency AC voltage has a slightly higher threshold voltage, the polarity is switched so quickly that the charges do not have time to accumulate at the interfaces, which in turn prevents surface fouling. As a result, low frequency AC actuation $(e . g .10 \mathrm{kHz})$ is the preferred method actuation method in that it is a good trade-off between maximizing droplet movement performance and minimizing surface fouling by minimizing hysteresis effects and allowing for a more reliable system.

\subsection{Integration in DMF}

DMF systems are commonly used for online analysis, in which samples in droplets are analyzed directly on the chip, and for off-line analysis where samples are collected and analyzed following removal from the chip.

Different detection methods have been coupled with DMF processes, including optical, electrochemical, and mass spectrometry (MS). Optical detection is the most widely used because it is easy to use, non-destructive to the sample, compatible with multiple wavelengths and generates rapid signal and readout. There have been several examples of coupling optical plate readers for absorbance detection with a light-emitting diode (LED) and a photodiode. For instance, Srinivasan et al. ${ }^{18,38}$ and Wijethunga et al. ${ }^{36}$ reported absorbance measurements of analyte in droplets. In a similar approach, fluorescence with a fluorimeter consisting of LED and photodiode ${ }^{37}$ and chemiluminescence with semiconductor-based thin-film optical detector ${ }^{38}$ have been 
employed to monitor DNA synthesis. The relatively short optical path lengths of DMF systems compared with conventional spectrometers ${ }^{29}$ however, is a limitation for measurements relying on optical detection. Electrochemical detection has also been integrated with DMF chips. For instance, Poulos et al. ${ }^{39}$ integrated on-chip thin-film $\mathrm{Ag} / \mathrm{AgCl}$ electrodes with the DMF droplet actuation for electrochemical detection of an artificial lipid bilayer array. As this technique was only able to detect the electrical properties of analyte species and is thus limited to electroactive species, there has also been great interest integrating DMF with MS due to its high sensitivity and the capability to separate and identify sample composition. Although much effort has been directed towards laboratory miniaturization, leading to the necessity of robust and reliable interfaces between MS and microfluidics, the general tools for wide spread use are not

yet available. ${ }^{40}$ As the technology is still immature, it is clear that analysis using the offline mode, is still the most widely used method with MS. Proteomics has been one of the most popular recent applications for MS resulting from the development of soft ionization sources like electrospray ionization (ESI) and matrix assisted laser desorption ionization (MALDI).

\subsection{Applications of DMF}

\subsubsection{Biofouling}

A significant challenge associated with applications of DMF is surface fouling, which usually occurs when moving droplets containing a complex mixture of biologics 
(e.g. cells) and hydrophobic molecules (lipids). Droplet constituents non-specifically adsorb onto Teflon ${ }^{\circledR}$-coated surfaces and both impede and reduce the fidelity of further droplet movement. DMF devices are rendered useless if droplets stick to the surface. Furthermore, sample droplets share the same path, which can result in crosscontamination and sample carryover. Several groups have developed methods to reduce surface fouling and prolong DMF device use through the use of oil medium, surfactants, and removable dielectric material. ${ }^{17,41-47}$ Several early reports by Fair's group ${ }^{7,17,38}$ have used an oil medium as a matrix to surround aqueous droplets. Suspending the droplets in oil prevents evaporation and reduces the surface energy, allowing droplet actuation with lower applied voltage. ${ }^{7,48}$ Nonetheless, applications that require oil-miscible solvents (i.e. organic solvents) or reagents, ${ }^{11}$ and drying of the droplets are incompatible with an oilimmersed system. ${ }^{13}$ Recently, Wheeler's group has reported the use of Pluronics, trademark name given to nonionic tri-block copolymers based on ethylene oxide and propylene oxide, and functions as an effective surfactant solution additive to reduce the sticky problem in DMF. ${ }^{47,49}$ Furthermore, they employed three types of removable skins as a dielectric layer ${ }^{50}$ to eliminate cross-contamination of sample droplets including polyethylene food-wrap sheets $(\sim 15 \mu \mathrm{m}$ thick $)$, clerical adhesive tape $(\sim 45 \mu \mathrm{m})$, and stretched sheets of wax film $(\sim 10 \mu \mathrm{m})$. Replacing the dielectric layer with each fresh of these materials prior to each assay enhanced the device longevity. In contrast to the use of an oil medium, these two methods are compatible with any type of droplets, and do not require special confinement methods to contain the oil. 
These advances and others have made DMF compatible with a wide range of applications including chemical and enzyme reactions, ${ }^{44,45,47,51,52}$ proteomic sample processing, ${ }^{5,46,49,53-57}$, cell assays, ${ }^{15,58}$ immunoassays, ${ }^{37,48,59,60}$ and medicinal and clinical diagnostics. ${ }^{6,10}$ These topics are reviewed below.

\subsubsection{Enzyme Assays}

The implementation of chemical and enzyme assays were the first popular application of DMF to evaluate substances of interest, to measure kinetics and to synthesize new compounds. Taniguchi et $a l .^{44}$ first studied the reaction of a bioluminescent assay by mixing droplets of luciferase with adenosine triphosphate (ATP). In another report, Srinibasan et al. ${ }^{51}$ demonstrated the clinical application of assays by performing a fully automated glucose assay in a range of human physiological fluids (serum, saliva, plasma, and urine) on a DMF device. In addition to quantifying substances of interest, Miller \& Wheeler ${ }^{45}$ developed the first pol-free DMF device to study enzyme kinetics and activity. As proof of principle, droplets of alkaline phosphatase (AP) and fluorescein diphosphate (FDP) were merged and mixed to form fluorescein on a multiplexed DMF device (Figure 1.7a). Enzyme reaction kinetics of AP generated by this DMF device were evaluated, and kinetic constants agreed with literature values. Recently, Fiddes et $a .^{47}$ also demonstrated the action of AP on FDP using cylindrical hydrogel discs incorporated in DMF devices. In this work, agarose gel discs were modified with AP, and droplets containing FDP were dispensed and merged into the gels for the cleavage of phosphate groups and the generation of fluorescein. Another attractive application of DMF is chemical synthesis of 
new compounds, which was first introduced by Wheeler group ${ }^{52}$ for control of multi-step reactions in parallel. The DMF device was used to carry out synchronized synthesis of five peptide macrocycles (Figure 1.7b), which in turn also demonstrated the potential for fast and automated synthesis of libraries of compounds for drug discovery applications.

a)

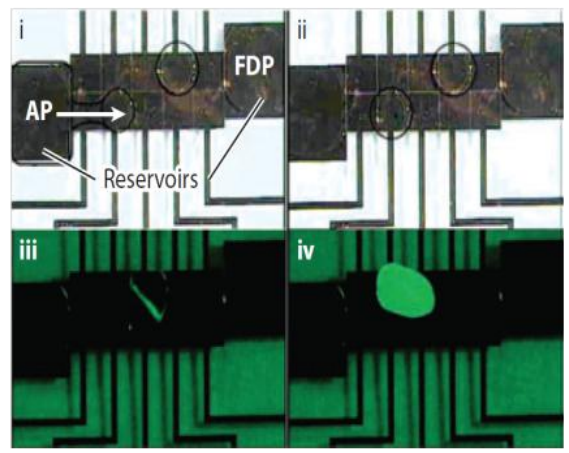

C)

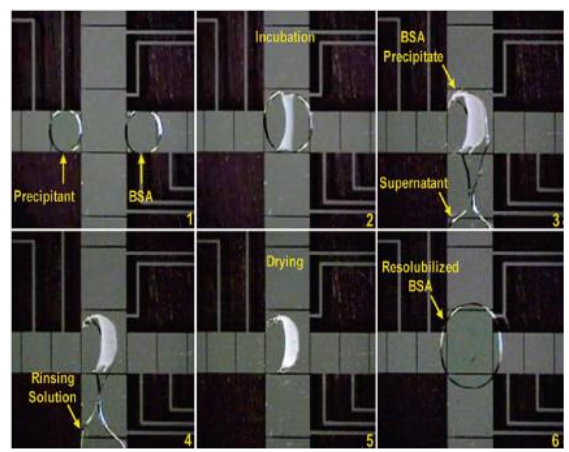

e)

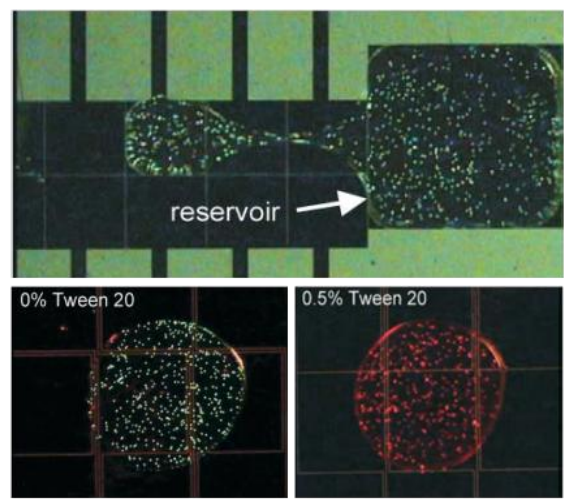

b)

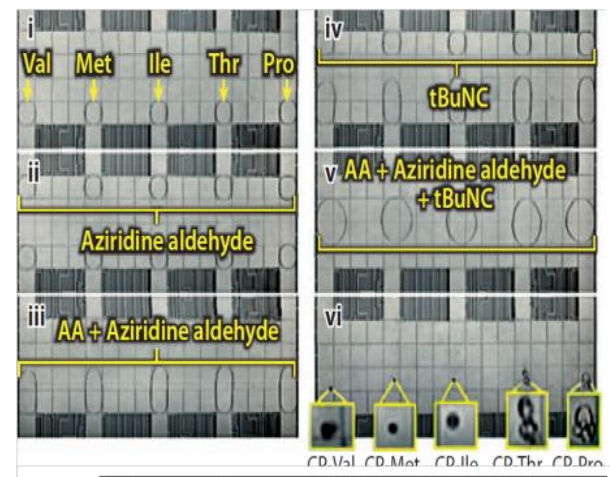

d)

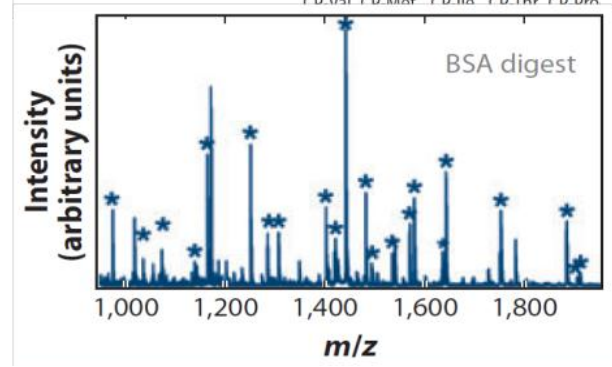

f)

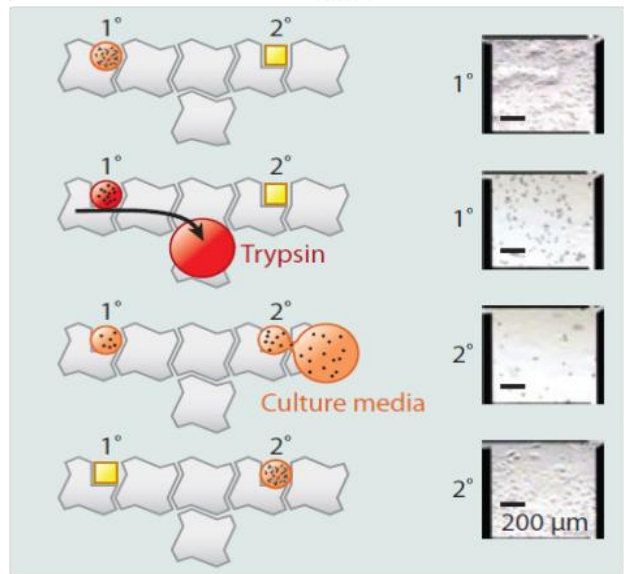

Figure 1.7 Applications of DMF ( $a$ ) Video sequence of an enzymatic assay. AP and FDP were dispensed from their respective reservoirs, merged, and actively mixed to form fluorescein, a product that is visible under fluorescent illumination. Reprinted with permission from ref. 60. Copyright 2008, American Chemical Society. (b) Video 
sequence of five parallel chemical synthesis reactions. Droplets containing different amino acids (AA) were merged with aziridine aldehyde and tert-butyl isocyanide $\left({ }^{t} \mathrm{BuNC}\right)$, and the reaction was allowed to proceed for $1 \mathrm{~h}$. The cyclic peptide (CP) products were isolated by allowing the solvent to evaporate. Reprinted with permission from ref. 52. Copyright 2010, Wiley. (c) Video sequence depicting the extraction and purification of proteins by precipitation. A droplet of bovine serum albumin (BSA) was merged with precipitant to form the protein precipitant on the device's surface. The precipitant was washed with rinsing solution and resolubilized in a droplet of borate buffer for subsequent processing. Reprinted with permission from ref. 5. Copyright 2008, American Chemical Society. (d) A representative MALDI-MS spectrum of BSA prepared by DMF-driven processing. Reprinted with permission from ref. 49. Copyright 2008, American Chemical Society. (e) Cell-based toxicity assays by DMF. (Top) A droplet carrying Jurkat T cells labeled with calcein AM (which fluoresces green) that are being dispensed from a reservoir. (Bottom) Droplets containing cells challenged with (left) $0 \%$ and (right) $0.5 \%$ Tween-20. Cells exposed to high concentrations of Tween 20 undergo necrosis and fluoresce red when labeled with ethidium homodimer 1 . Reproduced from ref. 58 with permission from the Royal Society of Chemistry. (f) (Left) Schematic and (right) pictures depicting a subculture of CHO-K1 cells in droplets by DMF. Reproduced from ref. 15 with permission from the Royal Society of Chemistry.

\subsubsection{Proteomics}

As proteomic sample processing requires multistep tedious workup before analysis by MS or other detectors, the capability of DMF to address multiple reagents and phases simultaneously makes DMF a good match for applications in proteomics. Jebrail et al. ${ }^{5}$ developed a DMF-based protocol for extracting and purifying proteins from serum by precipitation, rinsing, and re-solubilization (Figure 1.7c). This method had comparable protein recovery efficiencies $(\geq 80 \%)$ relative to conventional techniques and has the advantage of not requiring centrifugation, in addition to faster extraction and purification. In other studies, Luk et $a .^{49}$ and Chatterjee et $a l{ }^{53}$ reported DMF based multi-step protein processing following protein extraction, including reduction, alkylation, and digestion sequentially on chip prior to off-chip analysis by MALDI-MS and were 
identified by a Mascot database search engine (Figure 1.7d). In related work, the Garrell and Kim groups at UCLA ${ }^{54,55}$ developed DMF-based methods to purify peptides and proteins from heterogeneous mixtures by combining with in situ analysis by MALDI-MS.

Nelson et $a .^{56}$ improved upon these techniques by integrating resistive heating and temperature sensing elements for straightforward integration with MALDI-MS. Jebrail et $a l .^{57}$ integrated many of these methods into an automated digital platform including protein precipitation, rinsing, re-solubilization, reduction, alkylation, and digestion. Finally, Luk et al. $^{46}$ integrated agarose discs $(\sim 2 \mathrm{~mm}$ diameter $)$ bearing immobilized enzymes (e.g., trypsin or pepsin) into DMF systems for digesting proteins. These represented important milestones for DMF-based sample purification.

\subsubsection{Cell-Based Assays}

As the reagents and cell media materials are often prohibitively expensive for high-throughput techniques, cell-based assays have become another increasingly popular application for DMF. Barbulovic-Nad et $a .^{58}$ conducted the first cell based assays on DMF. In this study, a toxicity-screening assay was reported in which droplets containing cultured Jurkat-T cells were merged with droplets containing different concentrations of the surfactant that is lethal to cells, Tween-20. The resulting droplets were subsequently mixed with droplets containing viability dyes, (Figure 1.7e) and analyzed using a fluorescence plate reader. The results indicated that DMF based cell assays were more sensitive than identical assays performed in well plates and no significant effects on cell vitality were seen. As techniques for cell culture on DMF are being studied, Barbulovic- 
Nad et al. ${ }^{15}$ demonstrated the first DMF platform capable of implementing all of the steps required for mammalian cell culture - cell seeding, growth, detachment, and re-seeding on a fresh surface (Figure 1.7f). This innovation has proven useful for on-chip culture, analysis and transfection of cells and has enabled the implementation of complete cell growth over multiple generations.

\subsubsection{Immunoassays}

Immunoassays have recently become another popular technique for implementation by DMF that exploits specific antibody-antigen interactions for the detection of relevant analytes. Rastogi \& $\mathrm{Velev}^{59}$ first reported immunoassays for $\mathrm{IgG}$ and ricin by quantifying the presence of antigen in the sample from the pattern indicated by the antibody-coated latex and the gold particles. In another study, Sista et al. ${ }^{48}$ developed a droplet-based magnetic bead immunoassay using DMF to detect insulin and Interleukin-6. Droplets containing magnetic beads bearing antibodies were merged and mixed with droplets containing known concentrations of analyte, and a permanent magnet was then applied to immobilize the beads from the supernatant. The supernatant was then removed and beads were resuspended and washed in a new buffer droplet, and the immobilized analyte was detected by chemiluminescence. The authors also demonstrated the clinical applicability of this technique by developing troponin I immunoassays using whole-blood samples ${ }^{37}$. Recently, Miller et al. ${ }^{60}$ developed the first surface-based DMF platform for immunoassay applications implemented without beads or magnets by using human IgG as a model analyte. This method relied on device 
surfaces modified with spots of capture antibody (Fc-specific anti-human IgG), which binds antigen to the droplet sample and was detected through the use of fluorescein isothiocyanate (FITC)-labeled anti-IgG using a fluorescence plate reader.

\subsubsection{Medicinal and Clinical Diagnostics}

DMF has also proven to be a useful tool for applications in medicine as it allows precise control over sample collection, sample preparation, analytical processing, and detection. For instance, Jebrail et al. ${ }^{10}$ recently demonstrated the use of DMF for the extraction and analysis amino acids (AA) taken from newborn dried blood spot (DBS) samples to diagnose genetic disorders. This new method is integrated with hybrid microfluidics and an integrated nano-ESI emitter for direct detection by MS, which results in faster and more efficient analysis techniques. In a similar report, Mousa et al. ${ }^{6}$ demonstrated clinical sample cleanup and extraction of estradiol from $1 \mu \mathrm{L}$ samples of breast tissue homogenate, as well as whole blood and serum using DMF, and the extracted sample was detected with liquid chromatography tandem-mass spectrometry (LC-MS/MS). The DMF method employed was 1000x smaller in sample size, and 20x faster than conventional extraction methods for the extraction of steroids. Thus, this technique has enabled the implementation of routine monitoring of estrogen levels for the diagnosis of early breast cancer. 


\subsection{Human Plasma Protein Depletion Project Overview}

Of all applications discussed above, proteomics and clinical diagnostics seem to be the most attractive targets for DMF, in which complex samples can be pretreated and analyzed on a single device. Recently, clinical proteomics has emerged as an important field for the discovery of disease biomarkers. In particular, researchers are now systematically searching the human plasma proteome for biomarkers that can be used to predict the risk of cancer or monitor the progression of disease. ${ }^{61}$ However, these efforts are hindered by the complexity of plasma, which has a proteome that spans 10 orders of magnitude in concentration. ${ }^{62}$ As such, biomarkers at low concentrations can be masked by highly abundant proteins (HAPs) such as Igs and HSA. ${ }^{63,64}$

To reduce the complexity of plasma, many proteomic workflows include a pretreatment procedure that depletes HAPs from the sample. ${ }^{65,66}$ These depletion procedures typically use affinity chromatography spin columns, which contain affinity ligands that bind to specific HAPs to remove them from the sample. ${ }^{67-73}$ Although affinity chromatography is a useful pre-treatment strategy, there are drawbacks that limit its effectiveness. First, chromatography is a labour intensive process, requiring many sample preparation steps (e.g. multiple fluid handling steps followed by centrifugation). Additionally, the depletion process requires at least a 10-fold dilution of the sample in an appropriate loading buffer. ${ }^{74}$ Furthermore, there is a risk of sample loss arising from protein degradation during the long pre-treatment procedure (30 minutes to 2 hours), post-extraction-concentration steps to counteract the sample dilution, and sample 
handling during transfer and aspiration. These limitations represent both a major source of variability and a bottleneck for clinical proteomics.

To address these limitations, some groups have explored the concept of miniaturizing affinity chromatography using microfluidics. ${ }^{75-77}$ Microfluidic affinity chromatography has the potential to speed up protein depletion, minimize sample dilution, and eliminate the need for centrifugation and trained personnel. In a recent example, Mckenzie et $_{\text {al. }}{ }^{78}$ demonstrated a pneumatically-driven microfluidic device that deplete $66-77 \%$ of IgG from a complex sample using protein $\mathrm{G}$ functionalized beads dried on the device surface. The work was focused on preventing false positives in IgM assays, and did not examine proteomic sample preparation. In parallel, many groups have developed microfluidic systems to conduct immunoassays. ${ }^{79}$ Analogous analyte capture concepts developed for microfluidic immunoassays can be similarly applied to HAP depletion. The difference being that in immunoassays, the unbound constituents are discarded, while in HAP depletion, the unbound constituents are preserved.

Several liquid actuation schemes have been explored for microfluidics $;{ }^{80}$ however, DMF has a number of potential advantages for HAP depletion. In DMF, discrete droplets are manipulated by electrostatic forces on an array of electrodes coated with a hydrophobic insulator. ${ }^{29}$ When a sequence of voltage is applied to the electrodes, droplets can be addressed individually and made to move, merge, mix, and dispense from sample/reagent reservoirs. ${ }^{16}$ Since droplet operations can be conveniently controlled, experimental conditions can be modified to alter the protein depletion time or implement 
multi-stage depletion using the same device design. ${ }^{65,81,82}$ DMF has been applied to several sample preparation/extraction strategies, including protein precipitation, ${ }^{83}$ reversed-phase ${ }^{84}$ and strong cation exchange ${ }^{85}$ solid phase extraction, and liquid-liquid extraction. $^{6,36}$ In addition, DMF has been implemented for magnetic bead-based immunoassays, ${ }^{86-89}$ in which an external magnet facilitates the separation of droplets from antibody-coated beads. To our knowledge, DMF has never been used as a proteomic preparation tool for HAP depletion.

We report here the development of a new protein depletion platform that relies on DMF for liquid handling and paramagnetic beads (coated with Protein A, Protein G and Anti-HSA antibodies) for removal of abundant proteins. This new device brings about enhancements to traditional chromatography spin columns or flow-based microfluidics platforms. First, this method is fully automated and does not require external agitation for mixing or centrifugation; after placing the sample in the device, no further manual intervention is required. Second, the device depletes proteins rapidly because of efficient bead/sample mixing during incubation ${ }^{87}$ (e.g. $\sim 9$ minutes is required to remove $95 \%$ of a $0.5 \mathrm{mg} / \mathrm{mL}$ protein from solution). Third, the device can be programmed to carry out various permutations of protein depletion, involving the simultaneous or sequential removal of HSA and IgG. Beads immobilized with different affinity ligands can be facilely introduced to deplete other HAPs. During magnetic separation there is minimal sample dilution ${ }^{86}$ and loss during operation, eliminating the need for additional preconcentration steps, which are typically necessary using conventional depletion methods. 
Finally, we propose that this has the potential for facile integration with other microfluidic proteomic processing techniques including reduction, alkylation, and digestion $^{46,53}$ and separations..$^{90}$

\subsection{Project Objectives}

This thesis describes the use of DMF for carrying out protein separations by means of immunochemistry and magnetic bead-based pull down techniques that we have developed in both the Oleschuk and Wheeler groups. These have been categorized into three chapters: Validation and characterization of manual controller system and prototype DMF devices (Chapter 2), Off-chip (in tube) optimization of protein depletion (Chapter 3), and On-chip protein depletion using an automated DMF platform in Dr. Aaron Wheeler's lab at the University of Toronto (Chapter 4). 


\section{Chapter 2 - Chip Device and Controller System Preparations}

The majority of DMF devices reported thus far have been application specific, where unique devices have been designed and fabricated for the specific requirements of each application. ${ }^{22}$ Furthermore, an electronic controller is often used to interface with DMF devices to control the droplet movements via application of a series of voltages to the electrode array. A sequence of movements can be self programmed using commercially available software that comes with the controller system. ${ }^{22}$ The customization inherent in the DMF device, in combination with the magnet and electronic controller, work to create a complete system that may be used for a variety of sample handling, and processing procedures for biochemical and proteomic applications.

In this chapter, preliminary work on DMF chip and electronic controller preparations will be discussed. One-plate electrowetting, DMF chip design, fabrication, and two-plate experiments are described below.

\subsection{Experimental}

\subsubsection{One-Plate Electrowetting}

To examine and verify the electrowetting principle, ${ }^{7,8}$ an electrowetting platform as illustrated in Figure 2.1 below was designed and fabricated. 


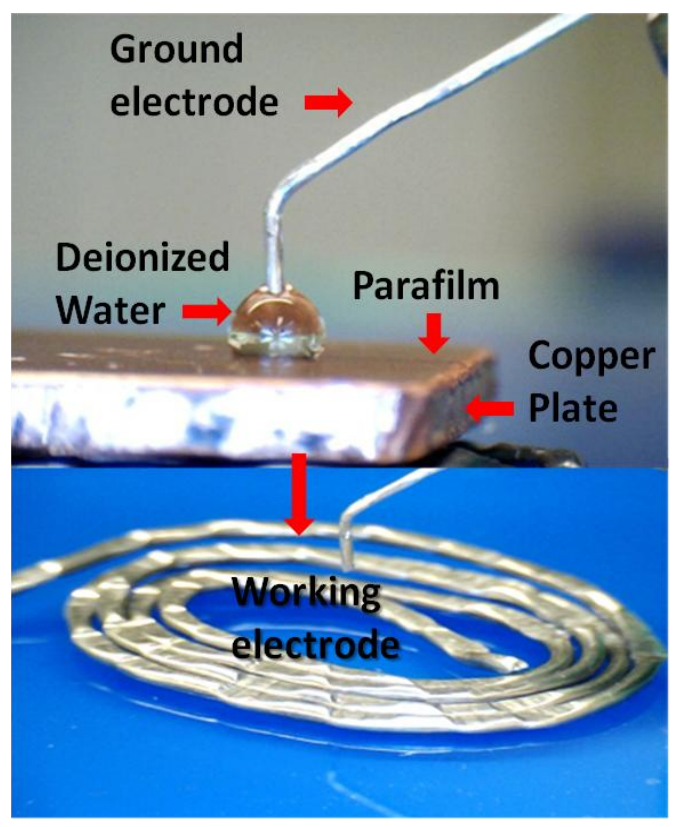

Figure 2.1 Electrowetting experimental setup.

The metal substrate used was a piece of copper plate obtained from the machine shop of Physics Department at Queen's University. The copper plate has dimensions of $3 \mathrm{~mm} \times 2 \mathrm{~cm} \times 2 \mathrm{~cm}$ (thickness $\mathrm{x}$ width $\mathrm{x}$ length), and features a polished surface. A sheet of Parafilm ${ }^{\circledR}$ was used to serve as both the dielectric and hydrophobic layer. ${ }^{91}$ The film was first stretched horizontally and vertically to its limits with thickness of $6-9 \mu \mathrm{m}$. (Sigma-Aldrich, Mississauga, ON) and then placed and adhered to the top of the copper substrate while ensuring there was no air gap between the film and the substrate. The copper substrate coated with Parafilm ${ }^{\circledR}$ was then placed in the oven for 1 minute at $70{ }^{\circ} \mathrm{C}$ in order to better adhere the film to the substrate. After cooling the substrate down to room temperature, a $2 \mu \mathrm{L}$ droplet of deionized water $\left(\mathrm{DI} \mathrm{H}_{2} \mathrm{O}\right)$ was dispensed from a 20 $\mu \mathrm{L}$ manual dispensing pipette to the surface of the copper substrate coated with Parafilm ${ }^{\circledR}$. An electrical HV controller, Microfluidic Tool Kit (Micralyne, Edmonton, AB) was used 
to supply DC potentials ranging from +200 to $+1200 \mathrm{~V}$. Driving DC potentials were applied to the wired loop working electrode attached to the bottom copper plate and the upper electrode was grounded at all times to $0 \mathrm{~V}$ and placed in the center of the droplet.

\subsection{PCB DMF Design and Fabrication}

PCB substrates were explored as a low cost platform for DMF devices. All PCB devices were designed as shown in (Figure 2.2a), based upon a glass chip design reported by Yang et al. ${ }^{84}$ All dimensions shown in Figure 2.2a are in micrometers. The design features an array of 86 actuation electrodes $(2200 \times 2200 \mu \mathrm{m})$ connected to six reservoir electrodes $(5000$ x $5000 \mu \mathrm{m})$, two large reservoir electrodes $(7500 \times 7500 \mu \mathrm{m})$, and interelectrode gaps of $50 \mu \mathrm{m}$. Multilayered flexible PCB devices were obtained from CMC Microsystems, which in turn were fabricated by Dupont. DuPont ${ }^{\mathrm{TM}}$ Pyralux ${ }^{\circledR}$ AP flexible circuit material is a double-sided, copper-clad laminate and an all-polyimide composite of polyimide film bonded to copper foil. ${ }^{92}$ These devices were flexible copper clads, and not coated with dielectric and hydrophobic layers. It is essential to coat a dielectric layer that is thicker than the metal layer to avoid direct contact of the liquid droplet with metal surfaces (electrolysis will happen otherwise). Since it is a multilayered material, electrode wires were connected either directly to contact pads to apply voltage on the upper side or through a connection to the underside of the PCB platform. A typical dielectric material used by the Wheeler group is Parylene $\mathrm{C}^{29}$ covered with an additional Teflon ${ }^{\circledR}$-AF layer to facilitate smooth droplet movement. These materials and instruments for the two coating steps were not available at Queen's University or CMC Microsystems; 
thus, coating was carried out at the Emerging Communications Technology Institute (ECTI), University of Toronto.

\subsubsection{PCB Device Coating Protocol}

Clean room reagents and supplies such as methanol and DI $\mathrm{H}_{2} \mathrm{O}$ were obtained from Fisher Scientific (Toronto, ON), Parylene C dimer was from Specialty Coating Systems (Indianapolis, IN), Teflon ${ }^{\circledR}-\mathrm{AF}$ was from DuPont (Wilmington, DE), and Fluorinet FC-40 was from Sigma-Aldrich (Oakville, ON). Briefly, all PCB devices bearing patterned copper electrodes (Figure 2.2a) were first cleaned with methanol followed by DI $\mathrm{H}_{2} \mathrm{O}$ and air-dried using a nitrogen gun. Prior to coating with Parylene-C and Teflon ${ }^{\circledR}-\mathrm{AF}$, the contact pads of each bottom-plate of the DMF devices were covered with low tack blue dicing tape squares (Semiconductor Equipment Corporation, Moorpark, CA) to avoid coating the contact pad areas.

The dielectric layer, Parylene-C, was applied using a vapour deposition instrument (Specialty Coating Systems, Figure 2.2b), and the hydrophobic layer, Teflon ${ }^{\circledR}$ AF, was spin-coated (1 wt \%/ wt in Fluorinet FC-40, $1000 \mathrm{rpm}, 30 \mathrm{~s}$, Figure 2.2c) followed by post-baking on a hot-plate $\left(160^{\circ} \mathrm{C}, 10 \mathrm{~min}\right)$. In addition to patterned devices, unpatterned ITO substrates (Delta Technologies Ltd., Stillwater, MN) were coated with Teflon ${ }^{\circledR}$-AF $(50 \mathrm{~nm})$. All thicknesses were measured with a profilometer in the clean room facility. The metal layer was measured to be $9 \mu \mathrm{m}$ and the deposited Parylene C layer was measured to be $12-14 \mu \mathrm{m}$. 

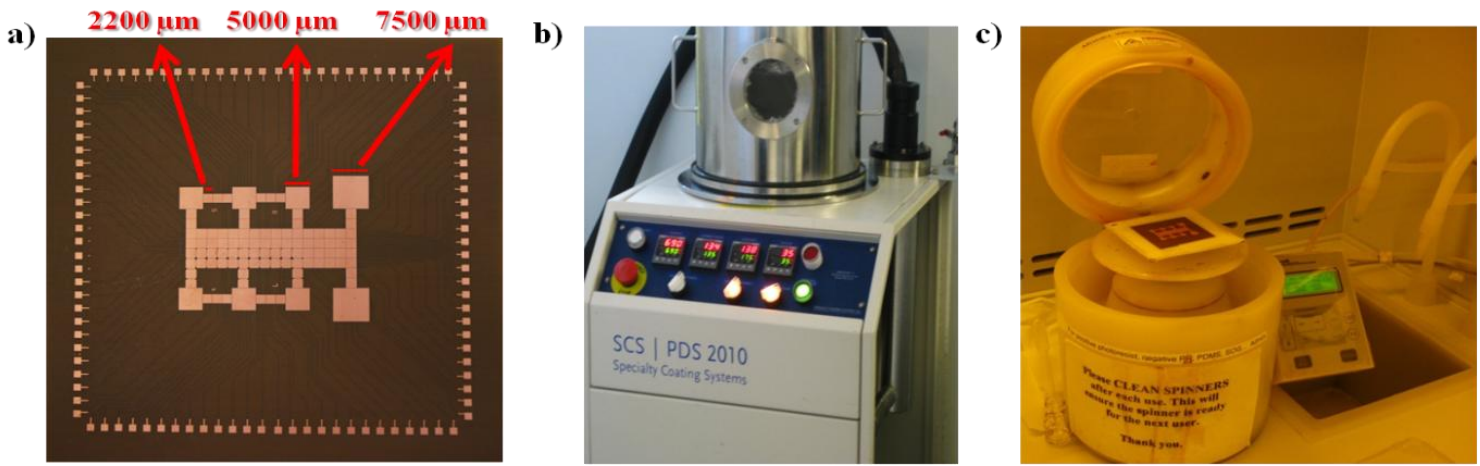

Figure 2.2 a) DMF chip using a PCB platform design is shown with all dimensions in $\mu \mathrm{m}$; b) Parylene-C vapor deposition coating system; c) Teflon ${ }^{\circledR}$-AF spin coating system

\subsection{Glass Chip Design and Fabrication}

Glass chips were fabricated based upon a design reported by by $\mathrm{Ng}$ et al. ${ }^{86}$ for immunochemistry and magnetic bead-based application as shown below (Figure 2.3).

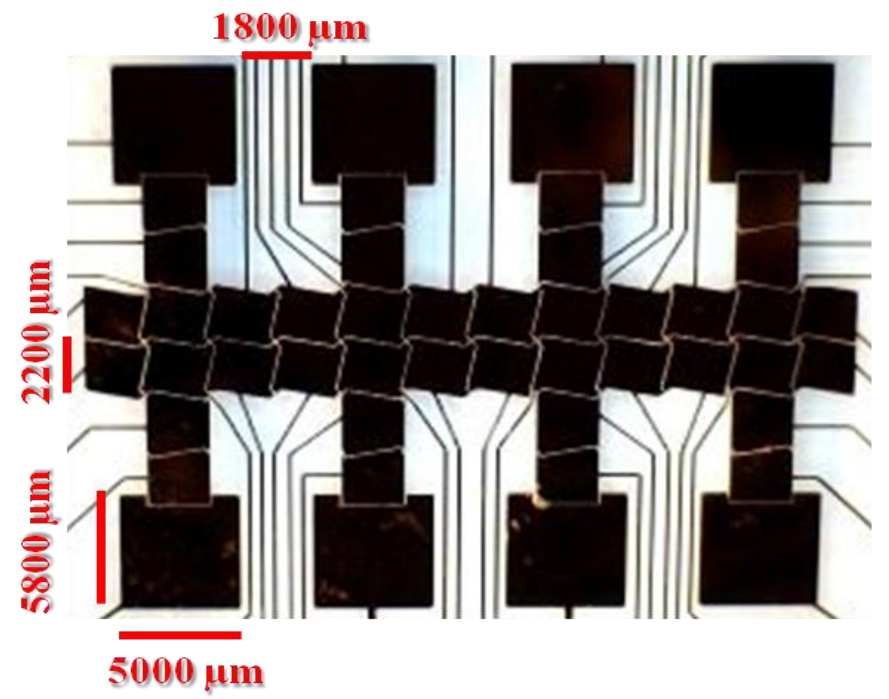

Figure 2.3 Glass chip design

The design features an array of 40 actuation electrodes $(2200$ x $2200 \mu \mathrm{m})$ connected to eight reservoir electrodes $(5800 \times 5000 \mu \mathrm{m})$, and inter electrode gaps of 50 
$\mu \mathrm{m}$ for small electrodes and $1800 \mu \mathrm{m}$ for large reservoir electrodes. Some initial prefabricated glass chips were also obtained from the Wheeler group in September 2012 and a second batch of glass chips prepared together with Wheeler group members in January 2013 were fabricated in the ECTI clean room facility.

Other than reagents and supplies (methanol, DI $\mathrm{H}_{2} \mathrm{O}$, Parylene $\mathrm{C}$, Teflon ${ }^{\circledR}$-AF and FC-40) mentioned above in section 2.2.2, clean room reagents and supplies for glass fabrication protocol also included MF-321 photoresist developer from Rohm and Haas (Marlborough, MA), CR-4 chromium etchant from Cyantek (Fremont, CA), AZ-300T photoresist stripper from AZ Electronic Materials (Somerville, NJ), acetic acid from Caledon (Georgetown, Ontario) and Silane A174 from Specialty Coating Systems (Indianapolis, IN).

To simplify our fabrication protocol, chromium- (200 nm thick) and photoresistcoated glass substrates $(2 ” \times 3 " \times 1.1 \mathrm{~mm})$ obtained from Telic Co. (Santa Clarita, CA) were exposed to UV light through a transparent photomask printed at 20,000 DPI (Pacific Arts and Designs Inc., Markham, ON) using a Suss MicroTec mask aligner (29.8 $\mathrm{mW} / \mathrm{cm}^{2}, 10$ seconds). The exposed substrates were developed in MF-321 for about 3 minutes followed by agitating and rinsing in $\mathrm{DI}_{2} \mathrm{O}$ and post-baked on a hot plate $\left(125^{\circ} \mathrm{C}, 1 \mathrm{~min}\right)$. The developed chromium substrates were etched in $\mathrm{CR}-4$ for about 3 minutes until complete removal of chromium, followed by rinsing in $\mathrm{DI} \mathrm{H}_{2} \mathrm{O}$. The remaining photoresist was stripped in AZ300T for about 5 minutes. After rinsing and drying, devices were coated with $\sim 7 \mu \mathrm{m}$ of Parylene $\mathrm{C}$ by depositing about $15 \mathrm{~g}$ of 
Parylene-C in the parylene coater and $200 \mathrm{~nm}$ of Teflon ${ }^{\circledR}-\mathrm{AF}$ (spin-coating, $1 \% \mathrm{w} / \mathrm{w}$ in Fluorinert FC-40, $2000 \mathrm{rpm}, 60 \mathrm{~s})$, and post-baked on a hot-plate (165 $\left.{ }^{\circ} \mathrm{C}, 10 \mathrm{~min}\right)$. The top-plates of DMF devices were formed by coating Teflon ${ }^{\circledR}-\mathrm{AF}(\sim 200 \mathrm{~nm}$, as described above in section 2.2.2) on unpatterned ITO-coated glass substrates.

Two types of dielectric layer materials were employed for DMF devices. Seven of our fabricated devices were coated with Parylene-C since the maximum batch for the coater is seven devices. The remaining five devices were spin coated with SU-8 3005 (Micro Chem, Massachusetts, USA) yielding a thickness of $8 \mu \mathrm{m}(2000 \mathrm{rpm}, 30 \mathrm{~s}$ ) followed by post-baking on a hot-plate $\left(95{ }^{\circ} \mathrm{C}, 10 \mathrm{~min}\right)$, according to the procedure

outlined in the product data sheet. ${ }^{93}$ Droplet actuation performance with devices bearing Parylene-C and SU-8 are discussed below.

\subsection{System Controller for Droplet Movement}

To actuate droplets on DMF devices, driving DC/AC potentials $\left(150-200 \mathrm{~V}_{\mathrm{pp}}\right)$ were generated by a NI-PXI chassis and a high-voltage amplifier (CMC Microsystems, Kingston, ON) that can amplify the output of the NI-PXI chassis controller by 50 times to a maximum of $200 \mathrm{~V}$, shown in Figure 2.4. The output of the amplifier is then connected to the two-plate DMF device. A 200x USB microscope (Veho, UK) was connected to the PXI-chassis and placed in front of the device to monitor and record the droplet movement in video and picture formats. The PXI chassis has integrated DC and AC power supply functions. DC potentials $(\sim 4 \mathrm{~V})$ were generated from the NI PXI-4130 function panel as 
shown in the Figure 2.5 by having two wires connected to the high voltage and ground pins of the PXI-4130 output. As shown in Figure 2.5 right, as the output enabled box is checked on the DC Power Soft Front Panel software, the output started generating potentials with the yellow light illuminated. AC potentials $\left(\sim 4 \mathrm{~V}_{\mathrm{RMS}}, 18 \mathrm{MHz}\right)$ were generated by NI PXI-5422 function generator with the yellow light on when the sine wave output is controlled by the FGEN Soft Front Panel software (Figure 2.6, right).

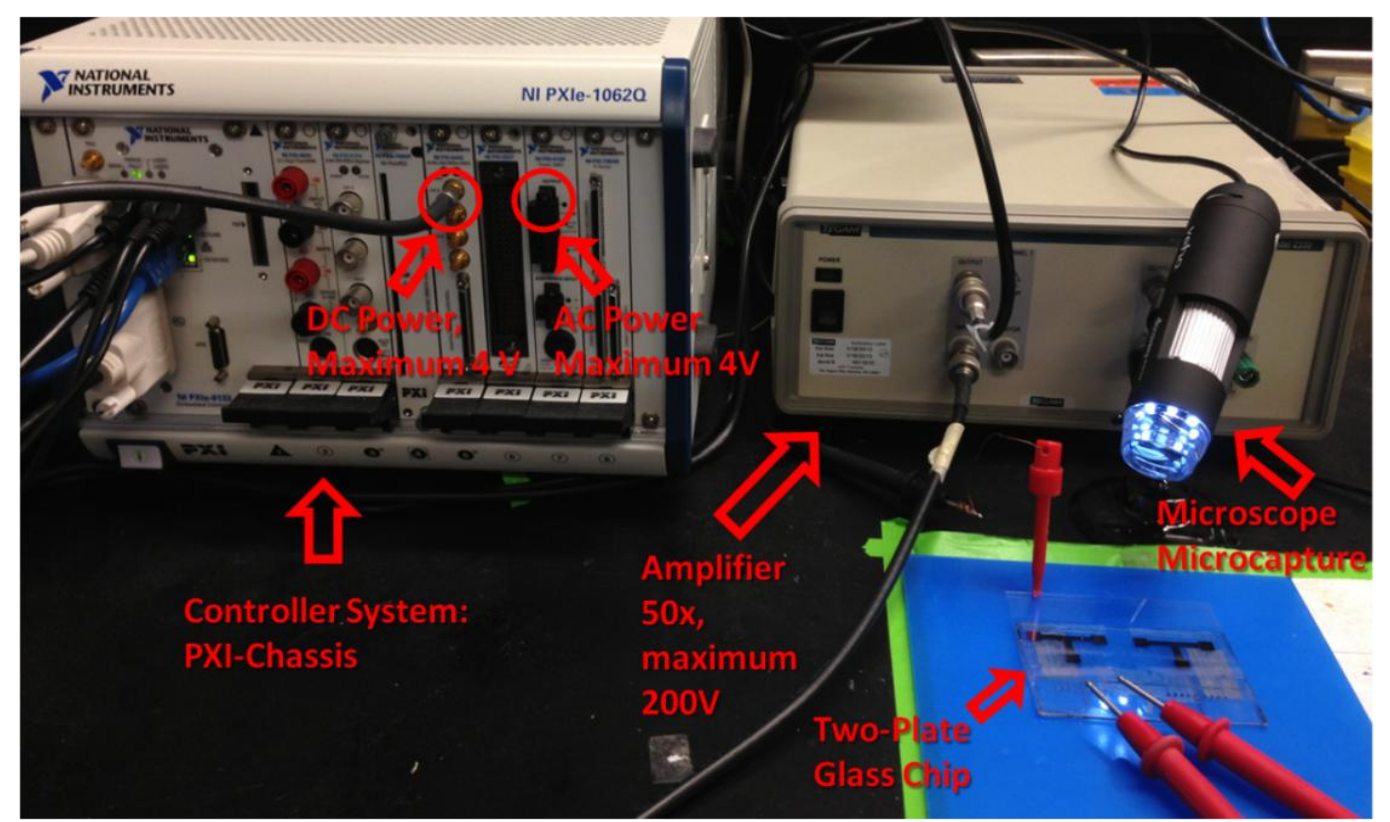

Figure 2.4 CMC Microsystems (Microsystem Integration Platform, MIP) system controller for microfluidic devices. 


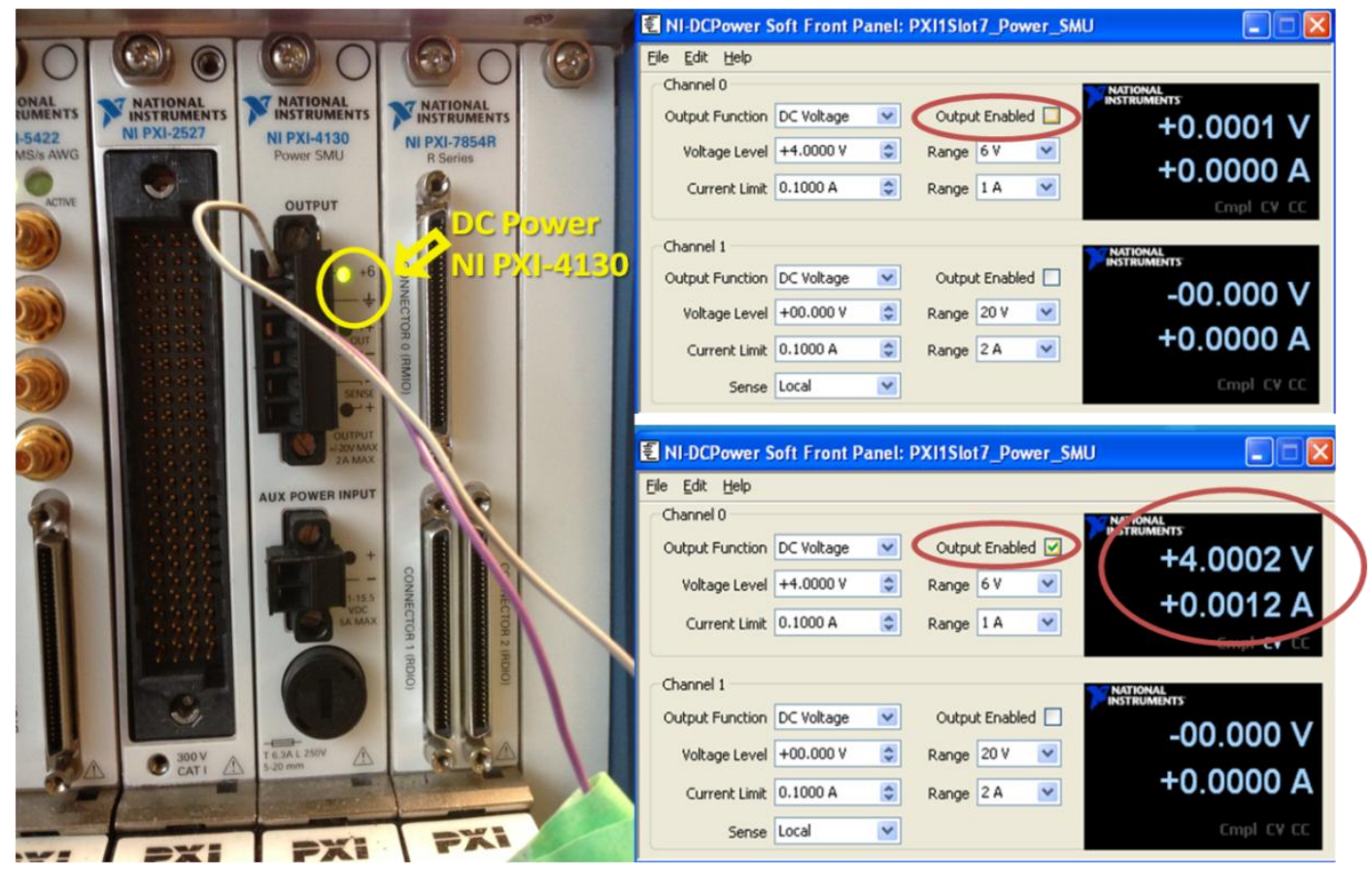

Figure 2.5 DCPower Soft Front Panel - software which controls the NI PXI-4130

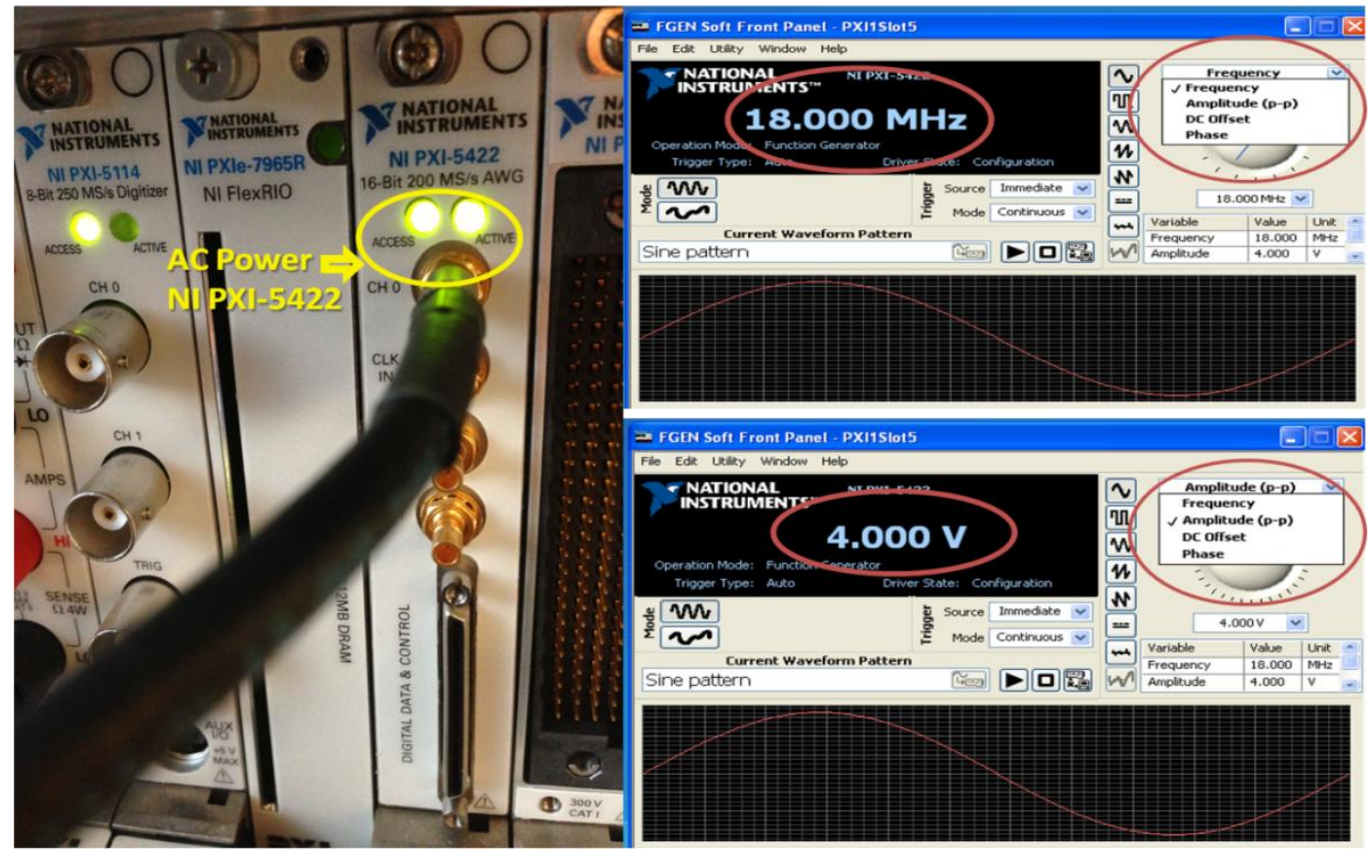

Figure 2.6 AC Function Generator Soft Front Panel - software which controls the NI PXI-5422 
As shown in Figure 2.7, the two-plate device was assembled with a spacer using two pieces of double-sided tape (3M, St. Paul, MN) which yields a distance of $180 \mu \mathrm{m}$ between a patterned bottom-plate and an unpatterned ITO glass top-plate. DC/AC voltage output was amplified to $200 \mathrm{~V}$ and applied to the patterned plate (via the exposed contact pads) while the top-plate is grounded with the help of an alligator clip (Figure 2.7). Water droplets of $2 \mu \mathrm{L}$ were dispensed from reservoirs to adjacent electrodes by actuating the high-voltage manually from the red probes to adjacent electrodes via contact pads as described previously. ${ }^{16}$

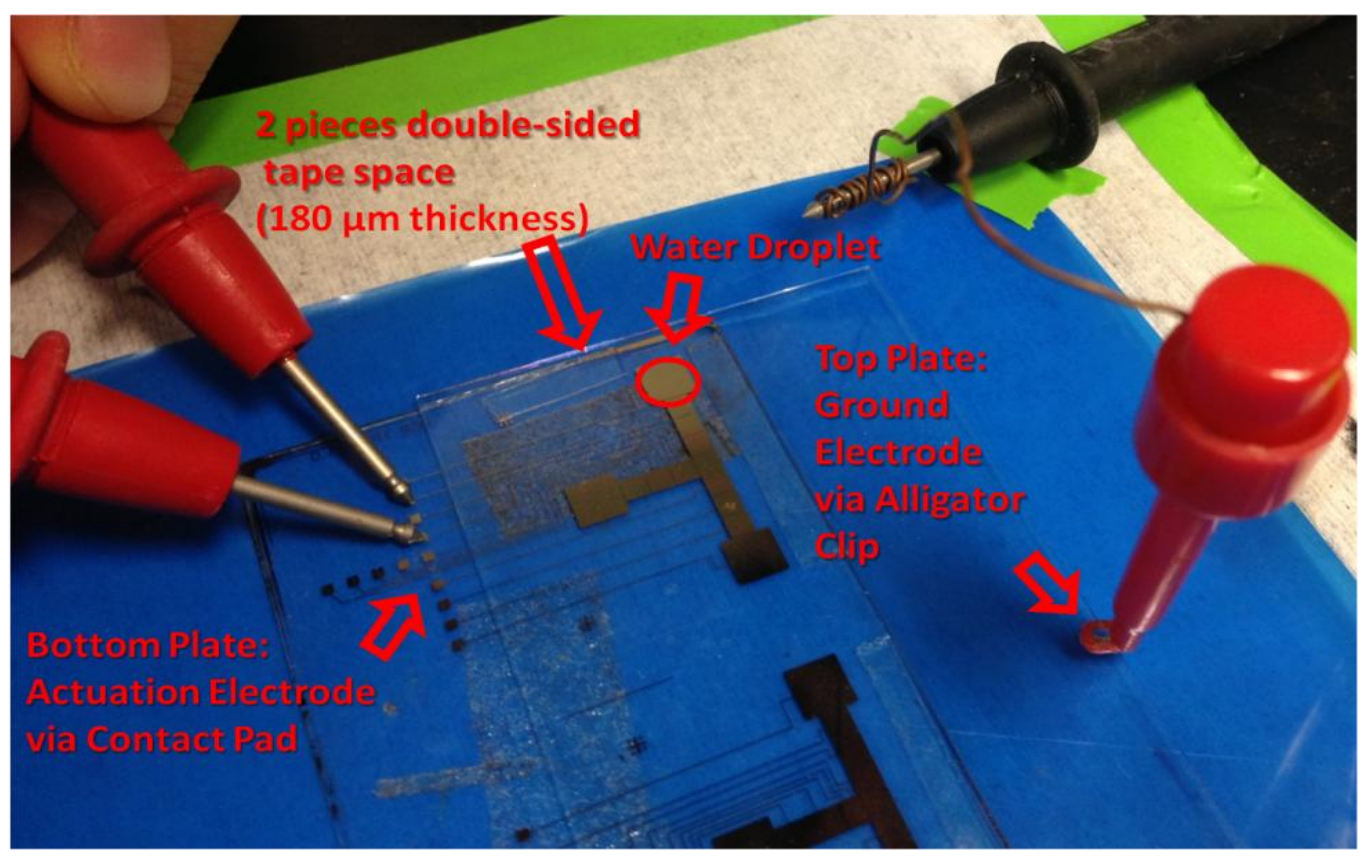

Figure 2.7 Manipulation of a droplet on a two-plate DMF device fabricated in the Wheeler group. Red voltage probes are placed in contact with the electrode pads to actuate the droplet. 


\subsection{Results and Discussion}

\subsubsection{Electrowetting Experiment}

Due to the equipment limitations and clean room accessibility, initial electrowetting experiments were performed using Parafilm ${ }^{\circledR}$ as a both a dielectric and hydrophobic material to replace Parylene C and Teflon ${ }^{\circledR}$-AF. A Microfluidic Tool Kit power supply was used because electrowetting on a one-plate device requires higher actuation potentials (>200V) and the CMC Microsystems controller generate a maximum of 200V. The electrowetting experiments were carried out by grounding the upper electrode and setting the high-voltage to the working electrodes while alternately turning on and off the high-voltage at five second intervals.

Different voltages required for fully stretched $(6-9 \mu \mathrm{m})$ and unstretched $(127 \mu \mathrm{m})$ Parafilm $^{\circledR}$ (dielectric and hydrophobic layers) were investigated. Electrowetting experiments with unstretched thicker Parafilm ${ }^{\circledR}$ required a much higher voltage (at least $600 \mathrm{~V})$ than with thinner stretched Parafilm ${ }^{\circledR}(200 \mathrm{~V})$, and this was expected since thicker dielectric layer requires in principle higher voltage as discussed in the section 1.3.2.

When a potential is applied, the contact angle is reduced as shown in Figure 2.8, which was expected according to the electrowetting principle. ${ }^{7,8,12}$ For the thickness of the Parafilm ${ }^{\circledR}$ that was manually stretched, larger contact angle change was observed with higher potentials. A minimum of $600 \mathrm{~V}$ was required to induce a contact angle change $\left(<10^{\circ}\right)$, but the higher the voltage, the larger the contact angle change (while increasing 
the voltage by increments of $100 \mathrm{~V}$ to a $1200 \mathrm{~V}$ maximum), and the largest contact angle change was observed at $1200 \mathrm{~V}$. The contact angle was measured using MB-Ruler software by aligning the base center of the ruler with the right base corner of the droplet captured from a video clip; the ruler reads the value of the angle automatically. Final contact angle measurements were obtained by subtracting the angle numbers read from MB-Ruler from $180^{\circ}$. As shown in Figure 2.8, the contact angle decreased from $84.9^{\circ}$ to $59.4^{\circ}$, which made a change of $25.5^{\circ}$.

Since the surface of Parafilm ${ }^{\circledR}$ is less hydrophobic, the water contact angle on Parafilm ${ }^{\circledR}$ is less than that on Teflon ${ }^{\circledR}-\mathrm{AF}\left(\approx 40^{\circ}\right) .{ }^{13}$
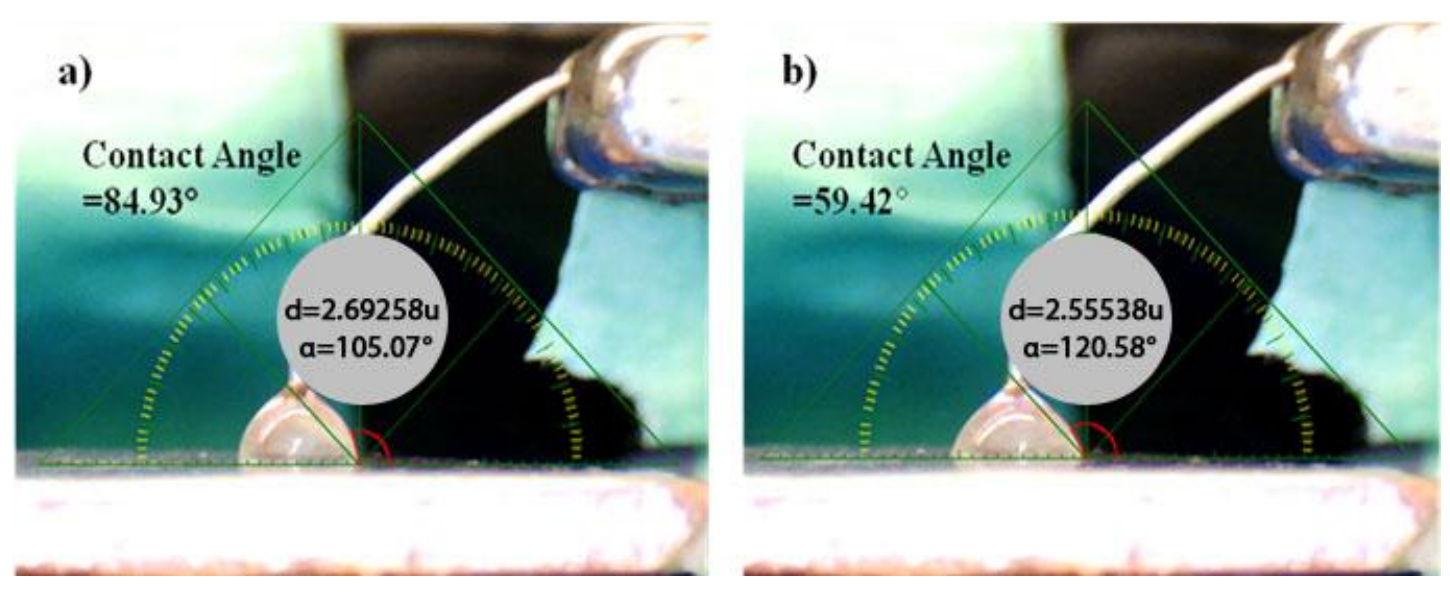

Figure 2.8 Screen shots from video clips of electrowetting a) before applying potential the contact angle is $84.9^{\circ}$, b) after applying potential, contact angle is reduced to $59.4^{\circ}$

\subsubsection{One-Plate Droplet Actuation}

In addition to electrowetting experiments, one-plate droplet actuation experiments were also conducted on the one-plate PCB and glass DMF devices, provided by both CMC Microsystems and the Wheeler group, using the Microfluidic Tool Kit since the actuation requires higher voltage than 200V. As shown in both Figures 2.9 and 2.10, 
when the electric potentials $(\sim 300 \mathrm{~V})$ were generated, there was a small droplet movement towards the next electrode, but not enough to displace the droplet completely from one electrode to another. Most of the time, the droplet moved into the inter-electrode space (between two electrodes), and stopped there. Both PCB and glass devices showed a similar trend when we tried to move the droplet without the second ITO layer on the top (two plate), even with higher potentials (>300V).
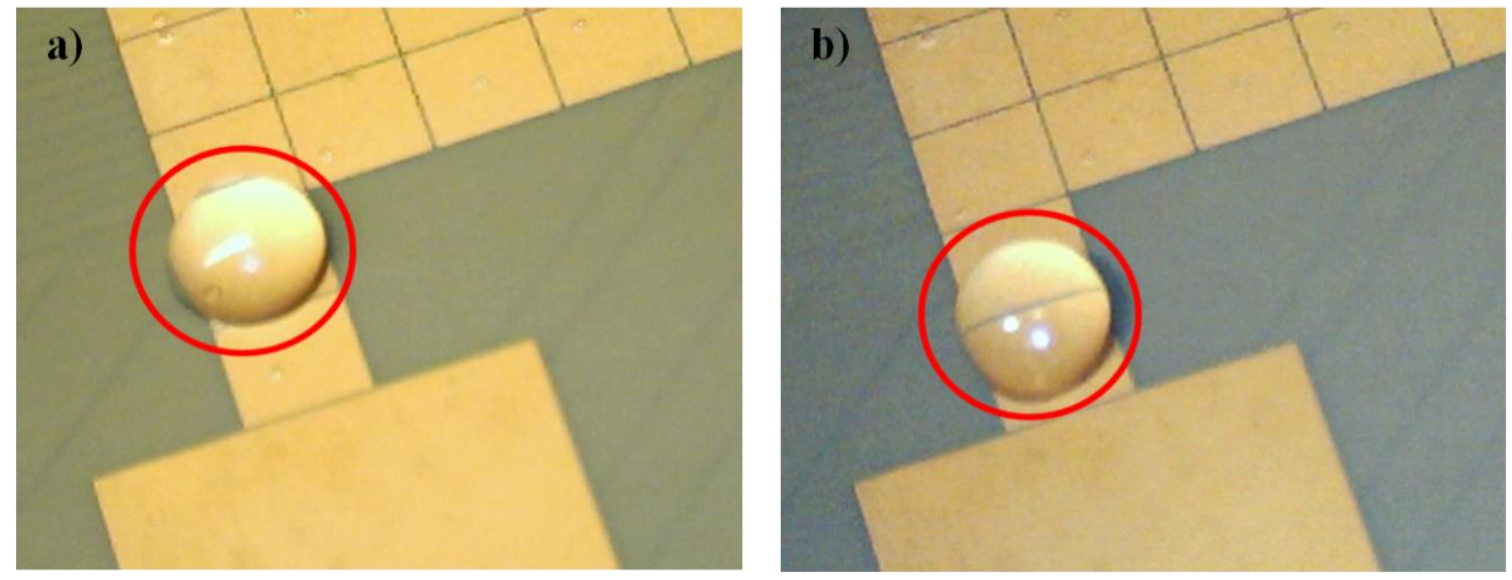

Figure 2.9 Captured images from video clips of droplet movement on PCB chip without ITO plate a) before applying potential, the droplet sits on one electrode b) after applying potential, droplet moved between the two electrodes. 

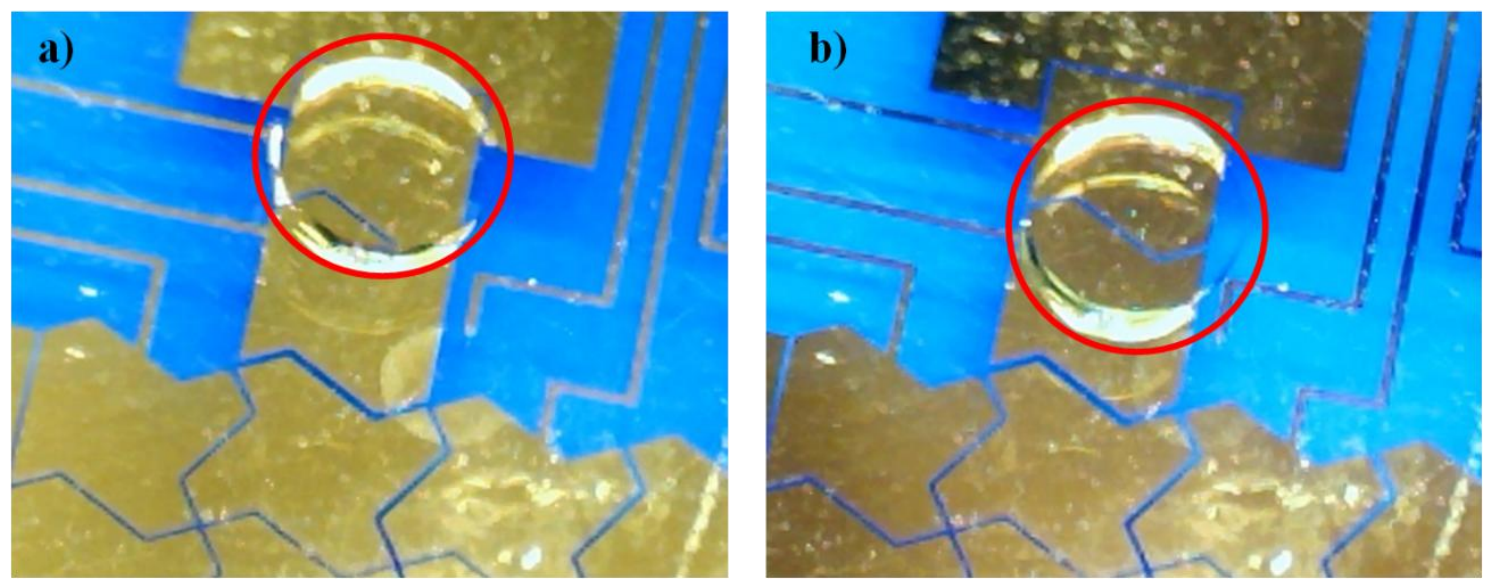

Figure 2.10 Images captured from video clips of droplet movement on glass chip without the ITO top plate (i.e. single plate) a) before applying potential, the droplet is positioned on a single electrode b) after applying potential, the droplet moved between the two electrodes.

\subsubsection{Two-Plate Droplet Movement}

For the two-plate DMF device, driving DC potentials ( 200V) were applied from the $\mathrm{CMC}$ controller system to electrodes on the bottom plate via the exposed contact pads and both the PCB and glass platforms were tested for actuation performance. For the PCB chip, the droplet remains immobile with the ITO top plate covered even when the electric potential is applied. However, for the glass chip, the same result (i.e. the droplet moved between the two electrodes) was observed for the one-plate DMF device as was observed for the two-plate glass DMF chip as shown in Figure 2.11. This effect could due to problems associated with the fabrication of the chips or possibly due to contamination after a long exposure to an environment outside of the clean room. 

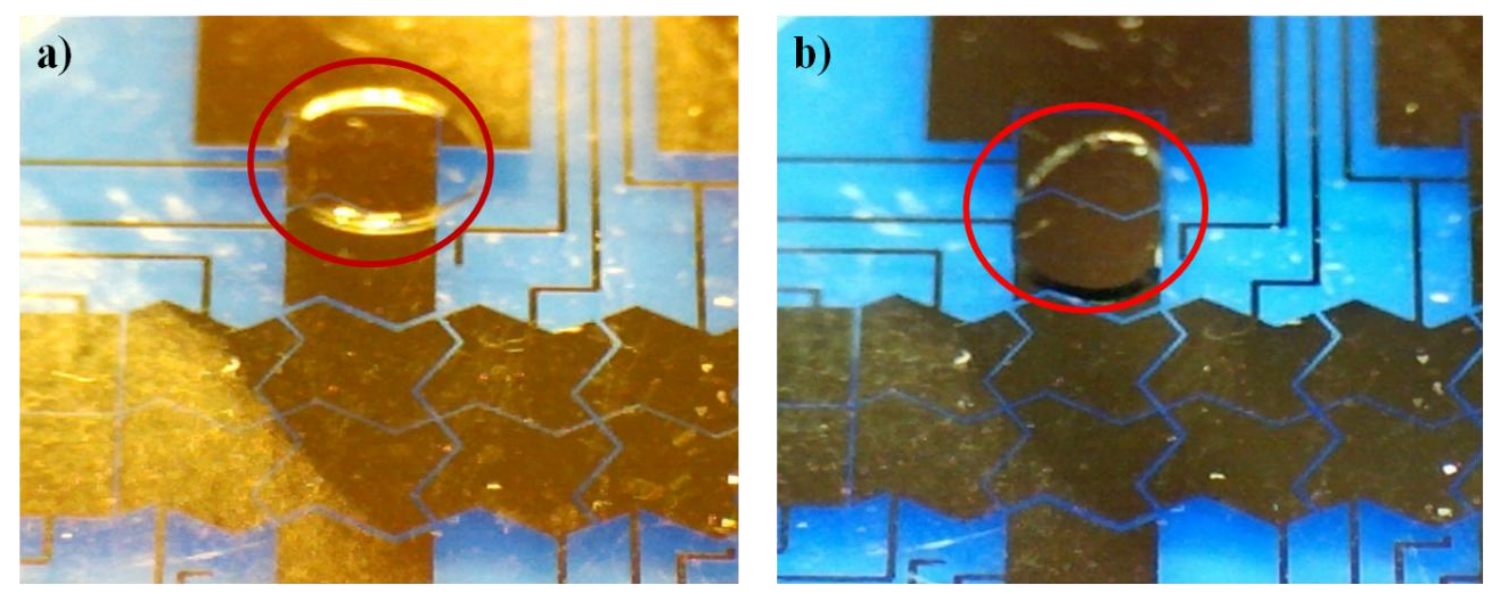

Figure 2.11 Screen captures from video of droplet movement on a glass chip a) before applying potential, the droplet is positioned on a single electrode b) after applying potential, the droplet moved between the two electrodes

\subsubsection{Testing Second Batch of Glass DMF Chips}

To test DMF devices, we moved magnetic beads solution with coated streptavidin provided by Innosep (Henan, Zhengzhou, China) across the device. Since our first batch of PCB chips and glass chips provided by the Wheeler group produced limited actuation, we fabricated a second batch of glass chips, half of them with Parylene-C and half with SU-8 as the dielectric coating, in the clean room facility at ECTI with Dr. Wheeler's PhD students. We realized that the second batch of chips with coated Parylene-C as the dielectric layer did not work as we expected. We noticed that as we assembled the top ITO plate with the bottom plate using the double-sided tape, the Parylene-C layer had a tendency to peel off from the chip (Figure 2.12). This effect was observed with all our second batch chips coated with Parylene-C. We later realized that this problem was most likely due to a step missing during the fabrication process as prior to Parylene-C coating process, DMF substrates are required to be primed in a silane solution (2-propanol, DI 
$\mathrm{H}_{2} \mathrm{O}$, A-174, and acetic acid 50:50:1:2 v/v/v/v, 10 minutes) and post-baked on a hot-plate $\left(80{ }^{\circ} \mathrm{C}, 10\right.$ minutes $)$.

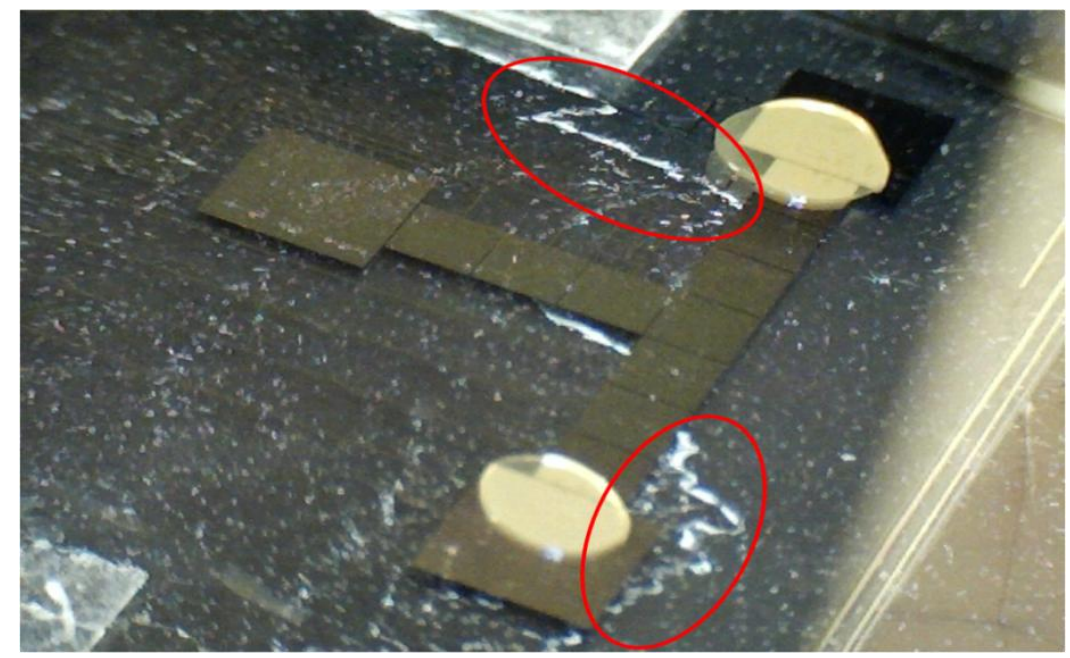

Figure 2.12 Non-functional glass DMF chip coated with Parylene-C with white scratch traces observed

Fortunately, glass chips coated with SU-8 as the dielectric worked properly after applying electrical potentials as the droplet moved smoothly in all four directions (up, down, right and left). Hence, we used these chips for application of magnetic beads on chip using manual controller system, which will be discussed in the next chapter. 


\section{Chapter 3 - Application of Protein Depletion Off-Chip and On-Chip Using Manual CMC Microsystems Integration Platforms (MIP)}

Preliminary protein depletion experiments were performed off-chip first in Eppendorf tubes followed by on-chip experiments on DMF devices manually to demonstrate the depletion of the high-abundance protein using magnetic beads. In this chapter, only IgG was used in the depletion experiments. Experiments in chapter 4 will

also include HSA. Since HSA and IgG account for $\sim 80 \%$ of total human serum, ${ }^{63}$ the inclusion of these proteins will make a more representative test case to evaluate on-chip depletion performance.

As illustrated in the schematic diagram below (Figure 3.1), the depletion process using DMF techniques comprises four steps. Initially droplets containing antibody immobilized paramagnetic beads and human plasma are dispensed on the DMF chip. The droplets are then uniformly mixed and incubated to deplete the high abundance proteins. Then a magnet is placed beneath the DMF device to immobilize the paramagnetic beads. Finally the fluid droplet containing the remaining unbound proteins is actuated away from the immobilized beads. 


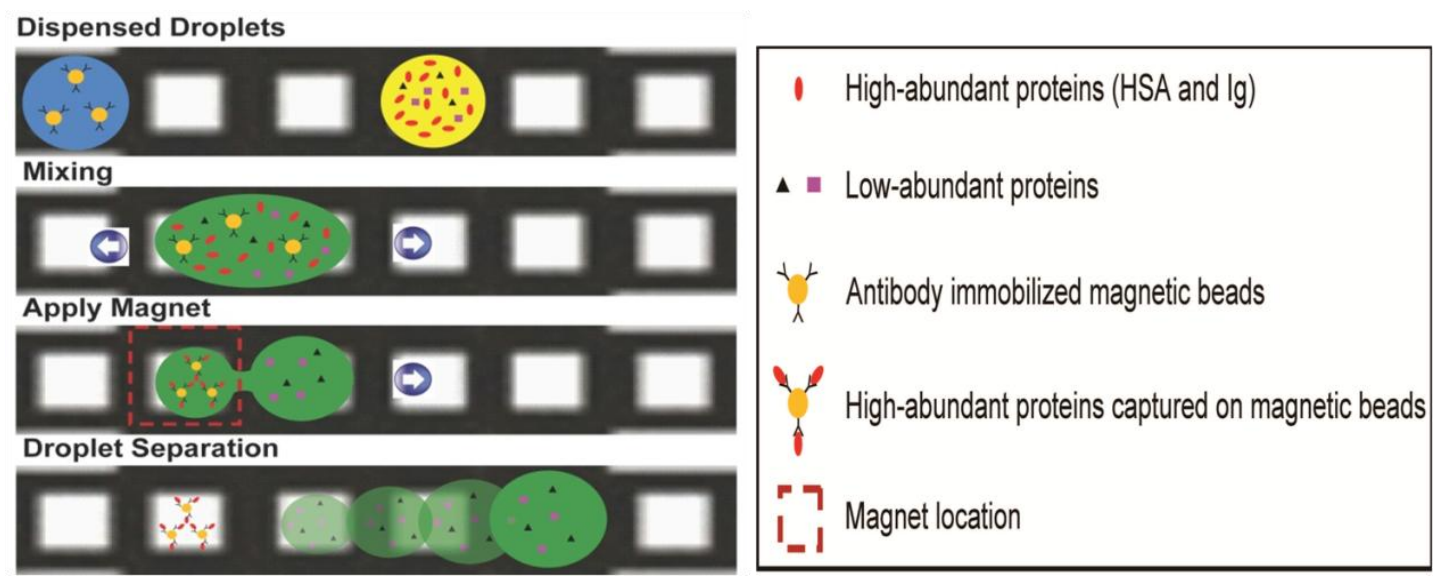

Figure 3.1 Schematics for magnetic bead-based separation of low-abundant proteins on a DMF platform.

\section{1 - Experimental}

\subsubsection{Reagents and Materials}

Unless otherwise noted, reagents were purchased from Sigma-Aldrich (Oakville, ON). DI $\mathrm{H}_{2} \mathrm{O}$ was utilized for all solution preparation and had a resistivity of $>18 \mathrm{M} \Omega \cdot \mathrm{cm}$ at $25{ }^{\circ} \mathrm{C}$. All protein and processing reagent solutions were prepared in working buffer (10 mM sodium phosphate, $\mathrm{Na}_{2} \mathrm{HPO}_{4}, \mathrm{pH} 7.0$ with $0.01 \%$ (w/v) Pluronic F-127 in water) prior to use. Eppendorf pipettes and tubes used were obtained from Eppendorf Canada (Missisauga, ON), and pieces of cylindrical shaped magnet(s) were obtained from Magnetic Materials Co. Ltd Dong Guan City Rui Xiang (Dongguan, Guangdong, China).

Reagent solutions used off-chip were prepared fresh in Eppendorf tubes. Solutions of human IgG were diluted from a stock concentration of $11 \mathrm{mg} / \mathrm{mL}$ purchased from Thermo Fischer Scientific (Pierce Antibodies, Nepean, Ontario) using the working buffer. Protein solutions of the representative low-abundance protein, transferring human, 
were made up from lyophilized solid in working buffer at stock concentration of 1 $\mathrm{mg} / \mathrm{mL}$. For the depletion of $\mathrm{IgG}$, Protein A/G MagBeads (Cat. \# L00277) from GenScript USA Inc. (Piscataway, NJ) were used. These beads have a binding capacity of more than $10 \mathrm{mg}$ Goat $\mathrm{IgG}$ per $1 \mathrm{~mL}$ settled beads. As the beads are supplied in $25 \%$ slurry, which implies that the beads are four times diluted, $1 \mathrm{~mL}$ settled beads would require $4 \mathrm{~mL}$ beads solution. The beads are super-paramagnetic beads of average $40 \mu \mathrm{m}$ in diameter, covalently coated with recombinant Protein A/G. Super-paramagnetic materials are a form of magnetic materials, which unlike ferromagnetic materials, do not retain any significant amount of magnetization in the absence of an externally applied

magnetic field, and thus do not form aggregates. In super paramagnetic state, an external magnetic field is able to align the ferrite particles and magnetize the nanoparticles. This locally increases the strength of the applied magnetic field. ${ }^{94}$

\subsubsection{Off-Chip Protein Depletion Protocol}

A bottle containing Protein A/G Magbeads was first vortexed for 10 seconds for complete resuspension. $100 \mu \mathrm{L}$ beads were then transferred into a clean $1000 \mu \mathrm{L}$ Eppendorf tube. The tube containing beads was placed on a piece of magnet to collect beads at tube wall and the supernatant was removed. The beads were washed with $1 \mathrm{~mL}$ working buffer where the buffer is mixed with beads for a minute. The magnet was then used to collect the beads and the supernatant was discarded. This step was repeated twice. Following $10 \mu \mathrm{L}$ of $\mathrm{IgG}$ from stock $(11 \mathrm{mg} / \mathrm{mL})$ and $10 \mu \mathrm{L}$ of transferring solution from prepared stock $(1 \mathrm{mg} / \mathrm{mL})$ were added to the tube containing the beads and the mixture 
was incubated at room temperature while mixing on a vortex stirrer for 30 minutes. After 30 minutes of mixing, a magnet was used again to separate the beads and the supernatant, and the latter was kept for MALDI-MS analysis.

MALDI matrix solution was prepared by dissolving $10 \mathrm{mg}$ of sinapinic acid (SA) in $1 \mathrm{~mL}$ of $50 \%$ ACN from Caledon (Georgetown (Halton Hills)), ON) in water containing $0.1 \%$ trifluoroacetic acid (TFA) from Thermo Scientific Pierce (Nepean, Ontario). Bovine serum albumin (BSA) obtained from Mann Research Laboratories (Port Saint Lucie, FL, USA) was used as the calibration standard at molecular weight of $66.5 \mathrm{kDa}$ for the analysis of all samples.

The protein supernatant collected was first acidified by adding 2\% TFA in 3:1 protein solution: $1 \%$ TFA solution ratio, and then matrix SA solution was added to the acidified mixture in $1: 1$ ratio. A $0.5 \mu \mathrm{L}$ aliquot of the matrix solution was first spotted on the MALDI 100-spot target plate and then left to air dry in the dark until complete dryness. A second layer of the mixture containing 1:1 matrix: acidified protein solution was then applied to the dried matrix spots on the target plate.

After the spots dried completely on the target plate, samples were analyzed using a PerSeptive Biosystems Voyager DE Pro MALDI-TOF Mass Spectrometer (AB Sciex, Framingham, MA, USA) operating over an m/z range of 20000-200000. A total of 250 shots were collected for each spectrum, with laser power adjusted to optimize the signal 
to noise ratio $(\mathrm{S} / \mathrm{N})$. Data were processed using baseline correction, resolution (set to 100), and smoothed (default settings) using Voyager Data Explorer software.

MALDI signal was dependent on the concentration of the samples used, especially for sample with large molecular weight such as IgG. Several samples were prepared by diluting the IgG solution at 1/2,1/5, 1/10,1/20,1/75, 1/100 and subjected to MALDI analysis to determine the optimum concentration range for detection of $\mathrm{IgG}$.

\subsubsection{On-Chip Protein Depletion Model Process}

On-chip protein depletion was performed manually using a PXI Chassis or MIP controller system, and droplet actuation was monitored and recorded using a microcapture USB camera. Magnetic beads (20 $\mu \mathrm{m}$ in diameter) functionalized with $\mathrm{COOH}$ and suspended in DI $\mathrm{H}_{2} \mathrm{O}$ were used to represent Protein $\mathrm{A} / \mathrm{G}$ beads and protein $\mathrm{IgG}$, respectively on chip to determine optimum droplet size, bead concentration and magnetic field strength. Magnetic beads coated with $\mathrm{COOH}$ were diluted five-fold by transferring $20 \mu \mathrm{L}$ of bead solution into a tube followed by removal of supernatant while beads are immobilized with a magnet. Following, $100 \mu \mathrm{L}$ of $\mathrm{DI} \mathrm{H}_{2} \mathrm{O}$ was added to dilute the beads. DMF droplet manipulation and magnetic separation for IgG depletion was carried out as illustrated in Figure 3.2. Eight separate steps were followed where AC potentials $\left(\sim 150 \mathrm{~V}_{\mathrm{RMS}}, 18 \mathrm{kHz}\right)$ are applied to each of the electrodes in the array: (1) Droplets containing sample and magnetic beads were first added into the reservoirs using pipettes. (2) A droplet containing the magnetic beads was dispensed from the reservoir

and separated from the diluent. (3) The magnet is engaged underneath the chip. (4) The 
magnetic beads are separated from the supernatant and supernatant is moved to the waste reservoir. (5) Droplet containing protein sample is dispensed and separated from the diluent. (6) Droplet containing protein is merged and mixed with magnetic beads manually, by moving the droplet left and right to adjacent electrodes continuously for five minutes. (7) Magnet is engaged underneath the chip. (8) Droplet depleted of protein is separated from the magnetic beads with the help of the magnet. 
1. Add sample and magnetic beads using pipette

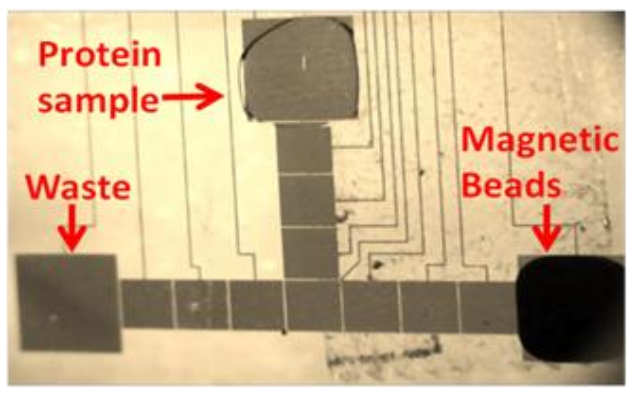

3. Engage magnet

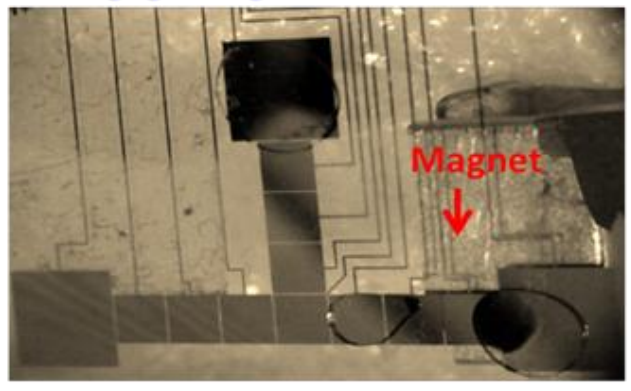

5. Dispense sample to magnetic beads

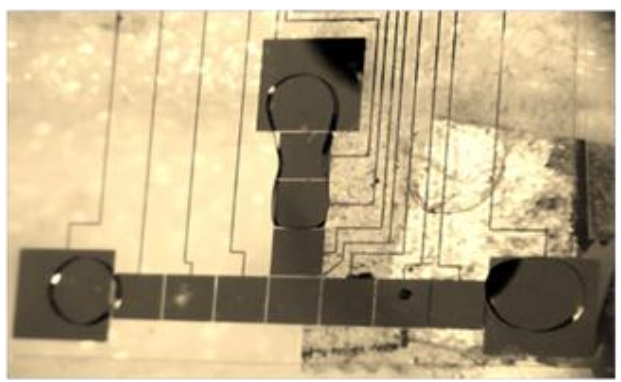

\section{Engage magnet}

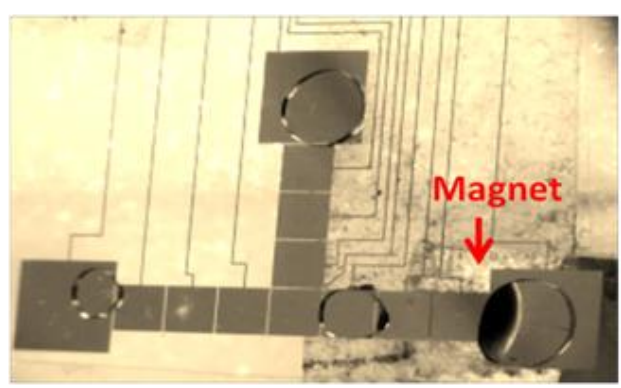

\section{Dispense magnetic beads}

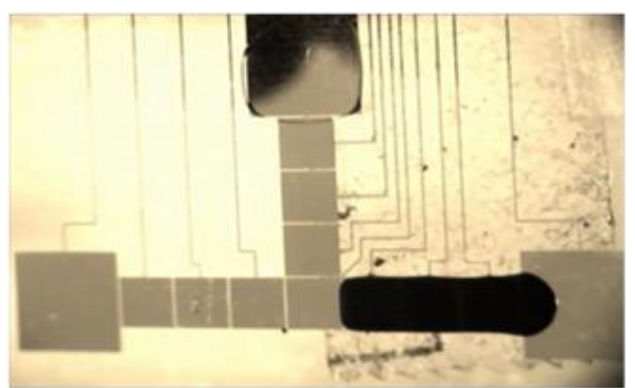

4. Remove supernatant from beads

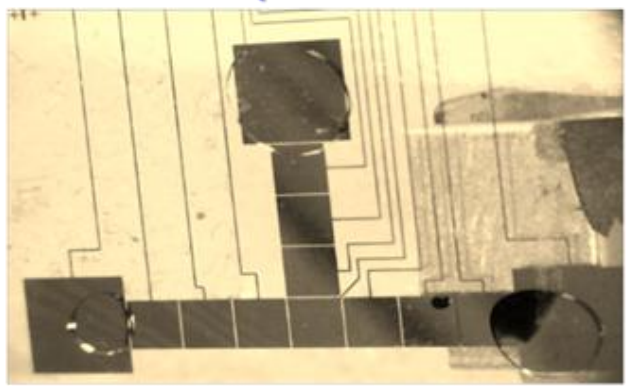

6. Mix beads and sample for five minutes

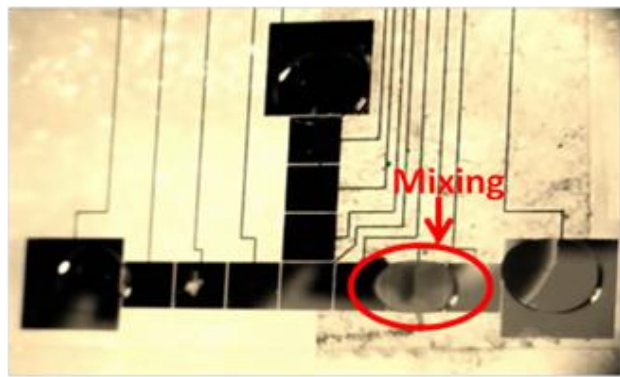

8. Separate extracted sample from beads

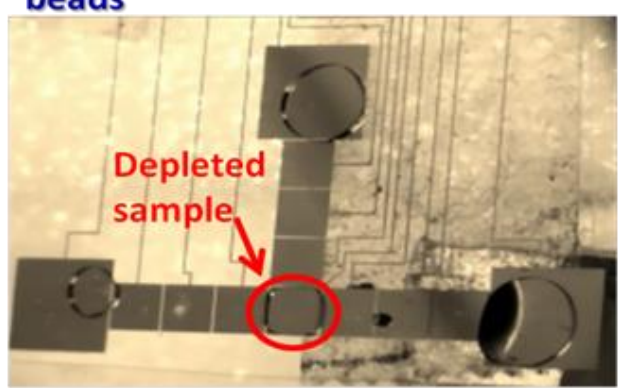

Figure 3.2 Images captured from a movie, which depicts the eight steps involved with the manual magnetic bead-based protein depletion process. 


\subsubsection{Magnetic-Actuated Droplet Manipulation}

In addition to DMF electrical based droplet manipulation, magnetic-actuated droplet manipulation can be performed on droplets containing suspended paramagnetic particles. Paramagnetic particles coated with $\mathrm{COOH}(5 \mathrm{x}$ dilution with water) were manipulated by hand by moving a magnet under a Teflon ${ }^{\circledR-}$ AF coated ITO glass slide. Two magnetic actuation experiments were conducted as illustrated in Figure 3.3: 1) droplet containing magnetic beads coated with $\mathrm{COOH}$ was moved with a magnet by hand; 2) magnet was then used to separate a droplet of water and magnetic particles from one another manually.

a) Droplet Transport on a Hydrophobic Surface

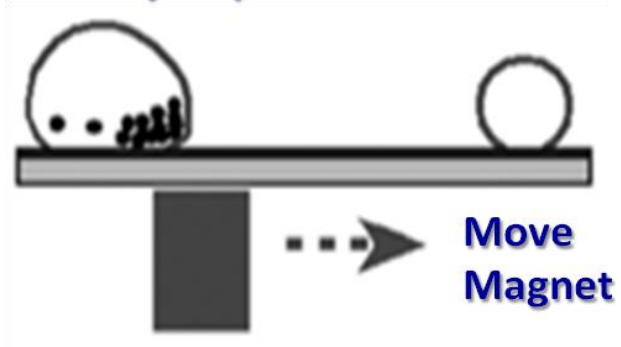

b) Separation of Droplet from Magnetic particles

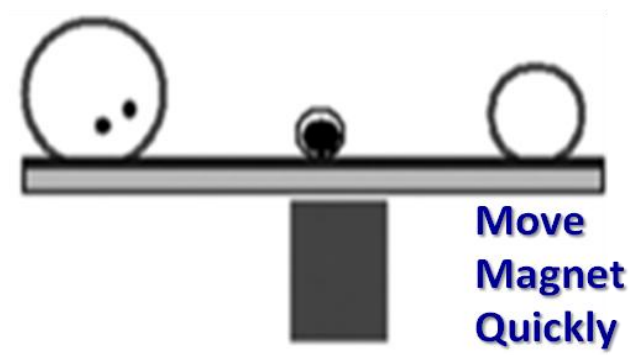

Figure 3.3 Magnet-actuated droplet manipulation a) Droplet transport on a hydrophobic surface b) Separation of droplet from magnetic particles. Reproduced with permission from ref. 95. Copyright 2009 The Royal Society of Chemistry.

\subsection{Results and Discussion}

The entire protein depletion process was first carried out in a $1000 \mu \mathrm{L}$ Eppendorf tube followed by MALDI-MS analysis. To investigate the efficiency of protein depletion in the tube, samples were analyzed by MALDI-MS for IgG before and after extraction 
with magnetic beads. To investigate the optimum concentration of $\operatorname{IgG}$ that gives the best signal level for the detection using MALDI-MS, a serial dilution of IgG at different concentrations was prepared. After a serial analysis using MALDI-MS for each concentration of $\mathrm{IgG}$, we found that $\mathrm{IgG}$ at $1 / 10$ dilution $(1.1 \mathrm{mg} / \mathrm{mL})$ produced a strong MALDI signal and we set this as the concentration of the high abundance protein for IgG within the sample. MALDI spectra were obtained for samples before and after depletion for $\mathrm{IgG}$ at a concentration of $1.1 \mathrm{mg} / \mathrm{mL}$. Conversely, the low-abundance protein, transferrin, was set at a concentration $10 \mathrm{x}$ less at $0.1 \mathrm{mg} / \mathrm{mL}$. In principle, if the protein IgG is removed quantitatively following extraction, the MALDI spectrum should show a single peak for transferrin. However, as shown in Figure 3.4, IgG signal levels were very comparable in samples a) before and b) after extraction with magnetic beads, which suggest that the binding of $\mathrm{IgG}$ to the Protein A/G beads was not quantitatively efficient. Similar results were observed when repeating the depletion process off line within an Eppendorf tube followed by MALDI-MS. It was suspected that the low binding was due to the presence of the surfactant, Pluronic F-127, that we added in buffer for smooth movement of protein on chip. To verify this hypothesis, the entire experiment was repeated without Pluronic F-127 in the working buffer. 
a) DMF Control

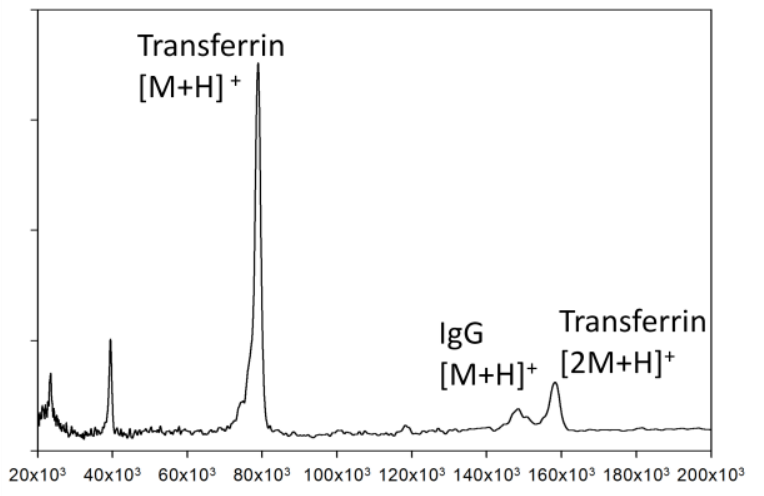

Mass $(\mathrm{m} / \mathrm{z})$ b) DMF Extracted

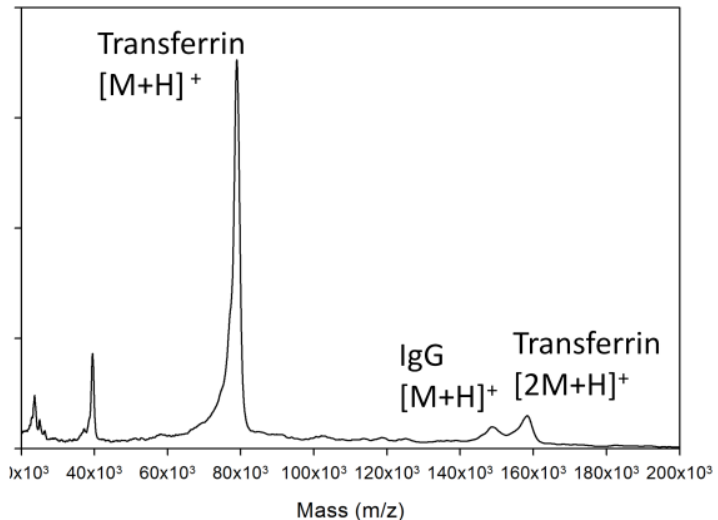

Figure 3.4 MALDI-MS Spectra for high-abundance and low-abundance proteins (IgG and transferrin) a) Before depletion b) After depletion

Surprisingly, the MALDI spectrum in Figure $3.5 \mathrm{~b}$ showed that the $\mathrm{IgG}$ signal decreased to a negligible level after extraction, when Pluronic F-127 was not present in the working buffer. This supported our hypothesis that the presence of Pluronic F-127 was interfering with the binding efficiency. The addition of Pluronics in protein sample is required for on-chip experiments to prevent nonspecific protein adsorption, as explained in the introduction, we decided to try other kinds of surfactant (Pluronic) instead of F127, and repeated the entire depletion process with Pluronic L64, L92, F68, and F88. Results showed that like Pluronic F-127, L64 also interfered with binding as its MALDI spectrum also showed small comparable signal for IgG after depletion just as depicted in Figure 3.4; whereas L92, F68 and F88 were found not to interfere with specific binding as no peak signal was detected for IgG from MALDI spectra after depletions. In addition, to find the Pluronic that could lead to the smoothest movement of droplet on DMF chip, we have also investigated on-chip performance of protein with the addition of either L92, F68 or F88. After testing each Pluronic on-chip F68 was found to work best on DMF chip. 
Therefore, we used Pluronic F-68 for on-chip protein depletion in Wheeler's lab, which will be discussed in details in Chapter 4.

a) With Pluronic F-127 Added

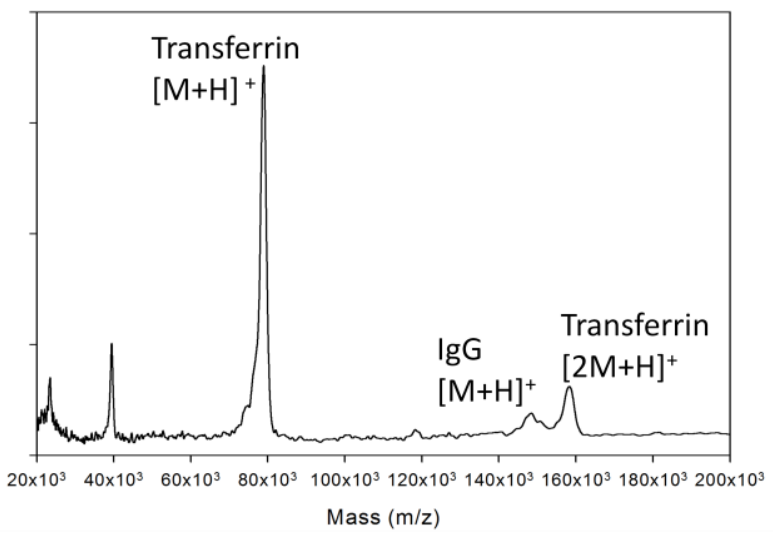

b) Without Pluronic F-127

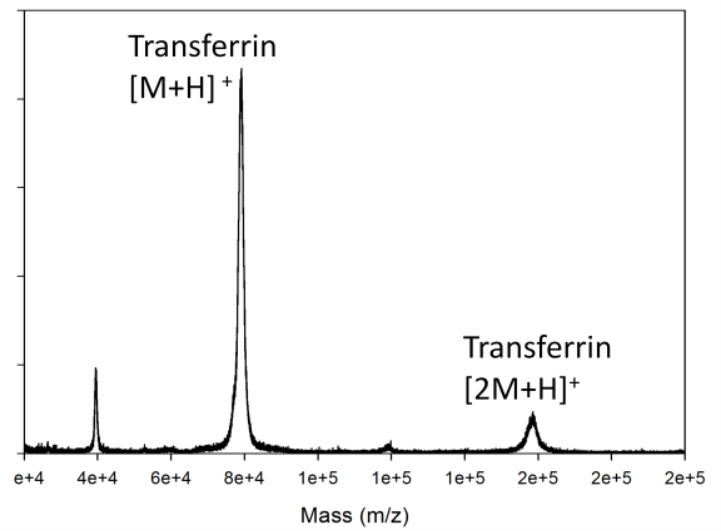

Figure 3.5 MALDI-MS Spectra for high-abundance and low-abundance proteins (IgG and transferring a) With Pluronic F-127 in the buffer b) Without Pluronic F-127 in the buffer

There was a risk that protein samples and magnetic beads would damage the two functioning chips without proper trials. As a result, we first used water and $\mathrm{COOH}$ functionalized magnetic beads to model the depletion process using a single device and then tested with the real reagents with the second device. As shown in Figure 3.2, the depletion process was successfully performed using a PXI (or MIP) controller system with water and magnetic beads. Following this, the real samples of $\operatorname{IgG}$ protein and Protein A/G magnetic beads were applied on-chip; however, this experiment was not successful. Protein A/G magnetic beads were not able to dispense uniformly since the size of beads (40 $\mu \mathrm{m}$ in diameter) was too large for DMF, and after several trials of dispensing with working buffer, the DMF chip no longer functioned properly. The problem was that the beads from GenScript were too large for the DMF devices. In 
previous trials droplets with magnetic beads $(20 \mu \mathrm{m}$ in diameter) we were able to be manipulated suggesting smaller beads produces better performance. As a result, we purchased different kinds of magnetic beads from Millipore (Billerica, MA) with diameters of $10 \mu \mathrm{m}$ (the details of these beads will be discussed in Chapter 4).

Because of difficulties associated with EWOD-based DMF such as protein adsorption on the DMF surface without Pluronic, limited device reliability, and complexity of the electrical controller system, we also proposed to manipulate droplet with magnetic-based actuation. As shown in Figure 3.6 below, a droplet containing magnetic particles was easily manipulated with magnet-actuation. Likewise, we also demonstrated in Figure 3.7 that magnetic beads were able to separate from the water droplet by holding the magnet underneath the hydrophobic platform and tilting the substrate to approximately 45 degrees.
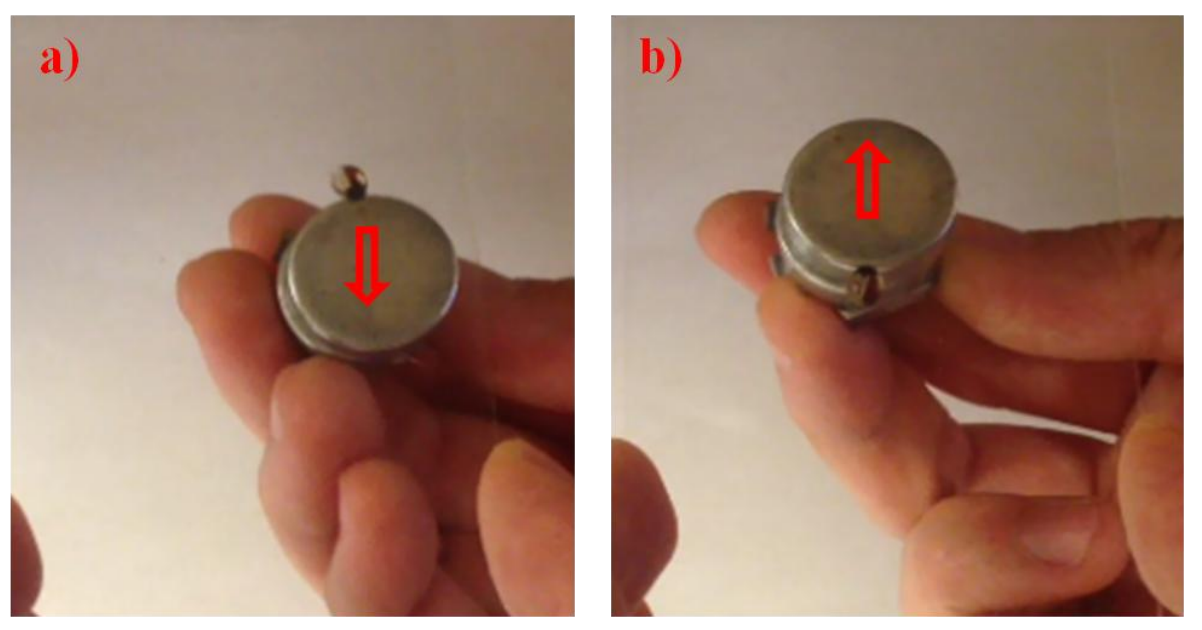

Figure 3.6 Magnetic actuated droplet containing paramagnetic particles moving back and fortha) Droplet moved downward b) Droplet moved upward 

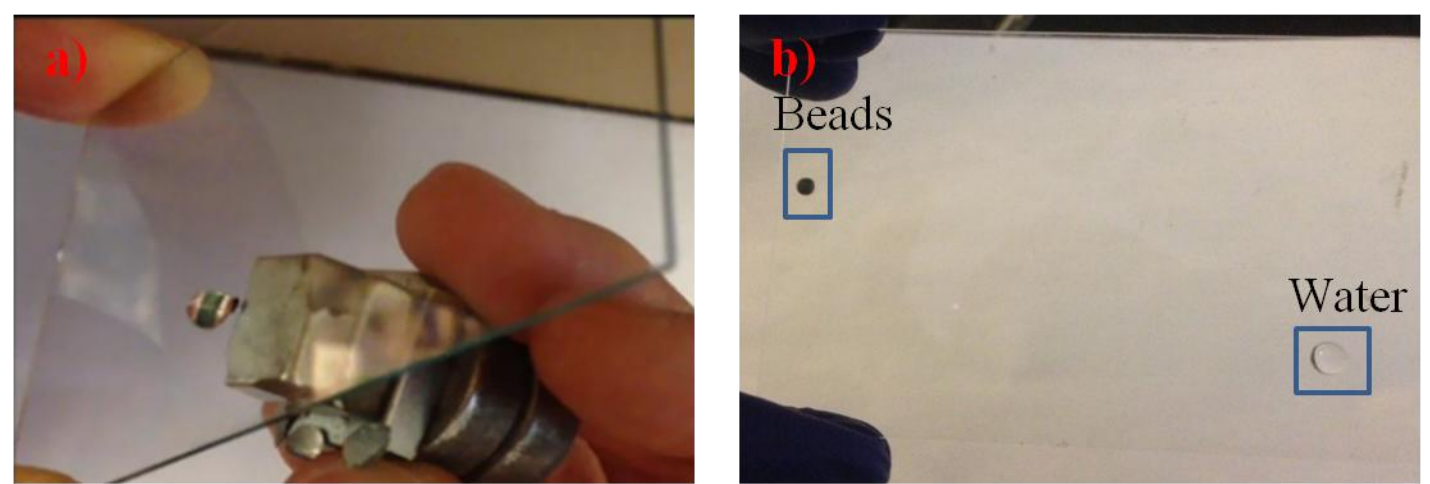

Figure 3.7 Separation of water droplet from magnetic particles a) Hydrophobic substrate tilted by 45 degrees b) Magnetic beads separated from water.

Since we ran out of DMF devices after many depletion trial experiments and the EWOD-based controller system and magnet-actuated droplet manipulation required actuation by hand, these limitations made our application of protein depletion on DMF difficult. Fortunately, we had the opportunity to collaborate with Dr. Aaron Wheeler and complete this project using his automated DMF platform for magnetic-particle-based immunoassays, which will be discussed in the next chapter. 


\section{Chapter 4 - Automated Protein Depletion On-Chip}

\subsection{Experimental}

\subsubsection{Reagents and Materials}

Unless otherwise noted, reagents were purchased from Sigma-Aldrich (Oakville, ON). DI $\mathrm{H}_{2} \mathrm{O}$ was utilized for all solution preparation and had a resistivity of $>18 \mathrm{M} \Omega \cdot \mathrm{cm}$ at $25{ }^{\circ} \mathrm{C}$. All protein and processing reagent solutions were prepared in working buffer (aqueous phosphate buffered saline, PBS, containing $1.5 \mathrm{mM} \mathrm{KH}_{2} \mathrm{PO}_{4}, 155 \mathrm{mM} \mathrm{NaCl}$ and $2.7 \mathrm{mM} \mathrm{Na}_{2} \mathrm{HPO}_{4}$ at $\mathrm{pH}$ 7.2, supplemented with $0.05 \%$ w/v Pluronic F-68) prior to use.

\subsubsection{On-Chip Protein Depletion Reagents}

Reagents used on-chip were prepared fresh in-house. Protein solutions of HSA (molecular weight based on amino acid composition of 66,437 Da) and representative low-abundance protein, hemopexin (molecular weight approx. 57,000 Da), were formed from lyophilized solid in PBS buffer, purchased from Life Technologies (Carlsbad, CA). Solutions of human IgG (molecular weight approx. 150,000 Da) were diluted from a stock concentration of $4.7 \mathrm{mg} / \mathrm{mL}$ in PBS buffer. Paramagnetic beads with specific functional coatings were obtained from Millipore (Billerica, MA). For the depletion of IgG and HSA, PureProteome ${ }^{\mathrm{TM}}$ Protein A/G Mix Magnetic Beads (LSKMAGAG02) and PureProteome ${ }^{\mathrm{TM}}$ Albumin Magnetic Beads (LSKMAGL02) were used, respectively. The 
paramagnetic beads are $10 \mu \mathrm{m}$ in diameter, and are coated with a mix of Proteins $\mathrm{A}$ and $\mathrm{G}$ and anti-HSA, respectively. Bead dilutions were performed by immobilizing the beads in a magnetic separation rack, removing the supernatant, and adding the desired volume of PBS. Two types of suspensions were used here: protein $A / G$ beads alone (at a dilution of 1:4 relative to the stock), or a mixture of protein $\mathrm{A} / \mathrm{G}$ beads (at a dilution of 1:4 relative to the stock) and anti-HAS beads (at a dilution of 1:2 relative to the stock). FITClabeled human $\mathrm{IgG}$ at $0.5 \mathrm{mg} / \mathrm{mL}$ was purchased from GenScript USA Inc. (Piscataway, NJ). FITC labeled HSA at $1 \mathrm{mg} / \mathrm{mL}$ was purchased from Abcam (Cambridge, MA).

\subsubsection{Off-Chip MALDI and LC-MS/MS Protein Depletion Analysis Reagents}

ZipTip ${ }^{\circledR}$ pipette tips, $\mathrm{C}_{4}$ and $\mathrm{C}_{18}$, and Milli-Q water were purchased from Millipore (Etobicoke, ON). Acetonitrile (ACN) was obtained from Caledon (Georgetown, ON) and TFA was purchased from Thermo Scientific Pierce (Nepean, Ontario). Both SA and $\alpha$-cyano-4-hydroxycinnamic acid ( $\alpha$-CHCA) MALDI matrix solutions were prepared by dissolving $10 \mathrm{mg}$ of solid matrix in $1 \mathrm{~mL}$ of $50: 50 \mathrm{ACN}$ : $\mathrm{DI} \mathrm{H}_{2} \mathrm{O}$ containing $0.1 \%$ TFA.

BSA obtained from Mann Research Laboratories (Port Saint Lucie, FL, USA) was used as the calibration standard for the analysis of all samples, except for the tryptic digested cytochrome $\mathrm{C}$ where a synthetic four peptide mixture (Angiotensin I at 1296.687 Da, Glu-fibrinogen at 1570.677 Da, renin at 1758.933 Da and ACTH at 2465.199 Da) was used for the calibration. 
Sequencing grade modified trypsin was purchased from Promega (Madison, WI, USA) and was used to digest cytochrome $\mathrm{C}\left(12\right.$ hours at $\left.37{ }^{\circ} \mathrm{C}\right)$, followed by quenching the reaction in $5 \%$ formic acid. ${ }^{96,97}$

\subsubsection{Device Fabrication and Operation}

DMF devices were fabricated in the University of Toronto Nanofabrication Centre (TNFC) clean room facility and were assembled as described previously (Figure 4.1A). ${ }^{86}$ An automated actuation system (described in detail elsewhere) ${ }^{87}$ was used to control droplet movement and magnet position for the immobilization of magnetic particles as shown in Figure 4.2. ${ }^{87}$ Droplet movement is controlled via custom Microdrop ${ }^{98}$ software (Figure 4.3) which was interfaced to the control system to engage a magnetic lens assembly. 
A

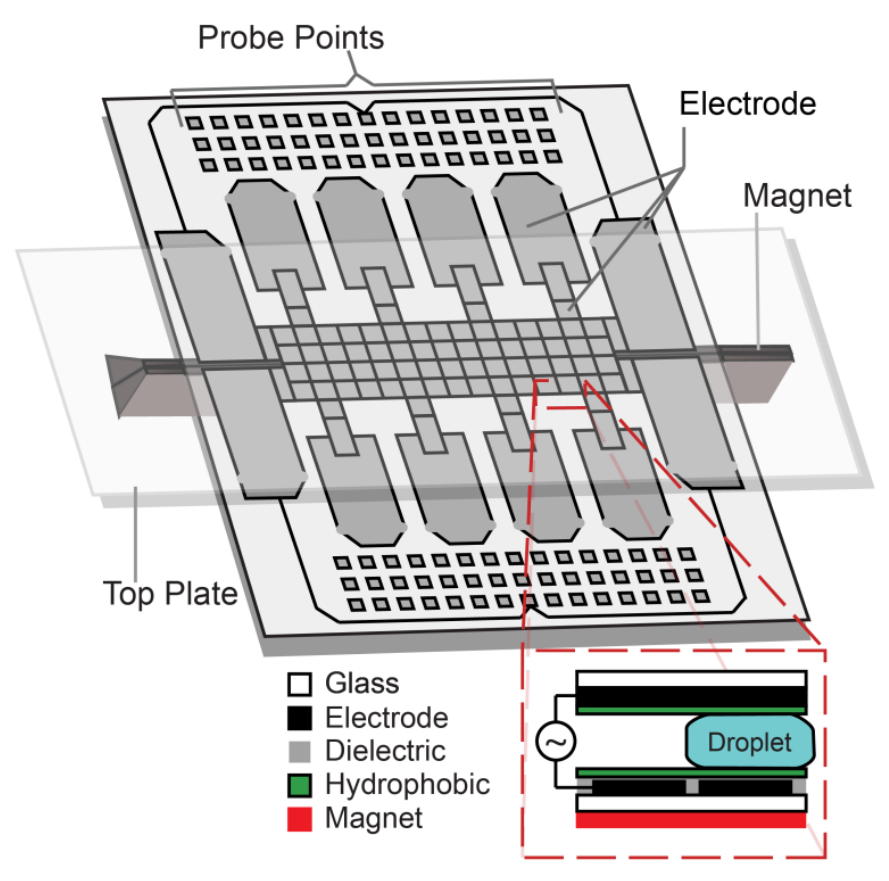

B

Immobilize beads, separate supernatant

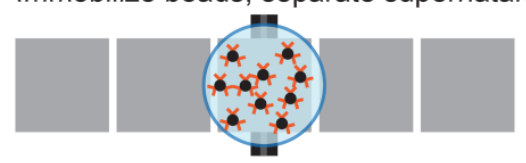

Mix beads with protein sample

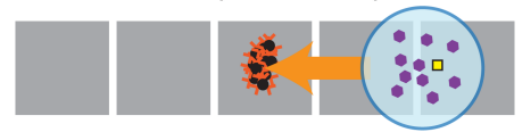

Immobilize again

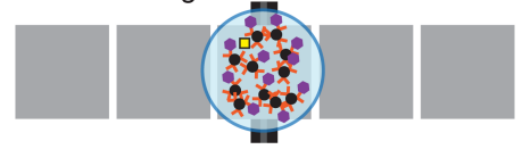

Separate depleted sample from beads

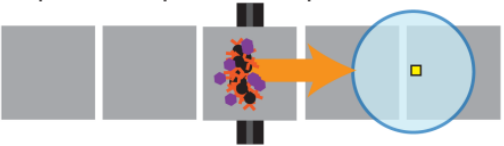

- High abundant protein

- Low abundant protein

* Functionalized magnetic bead

- Captured protein on bead

Magnet

Figure 4.1 Device and Processing scheme. A) Schematic representation of DMF device used for protein depletion in the automated magnetic separation system. Inset shows a cross-section of the device layers when the magnet is in position for magnetic separation. B) Schematic representation of protein depletion using magnetic beads and DMF. First, functionalized magnetic beads are isolated from their supernatant by magnetic separation. Second, protein samples are added to the magnetic beads and mixed. Third, application of a magnetic field immobilizes the beads again. Fourth, the immobilized beads are separated from the depleted protein solution by DMF actuation and the depleted sample is ready for analysis. 


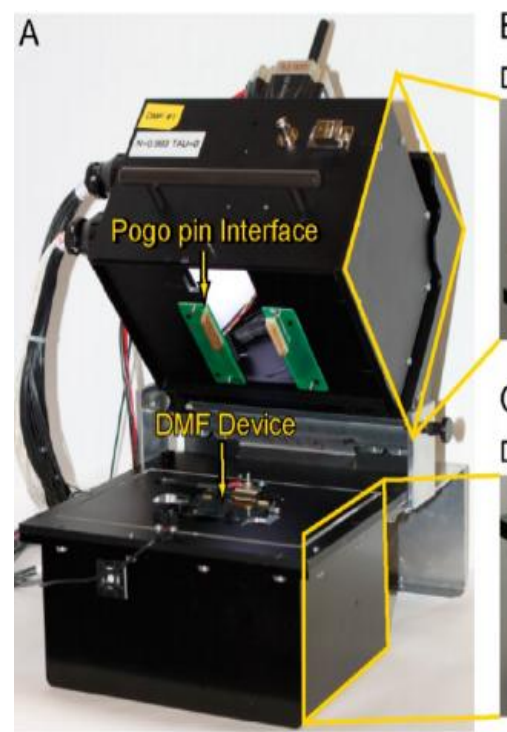

B

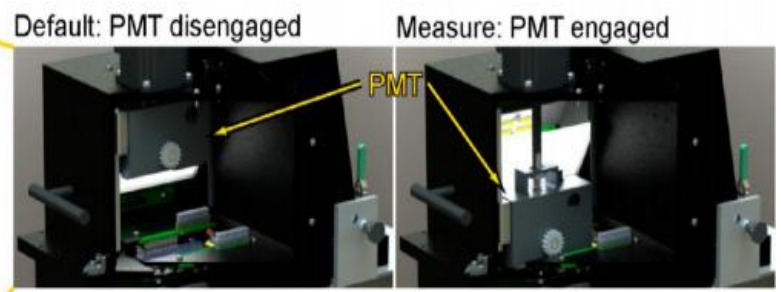

C

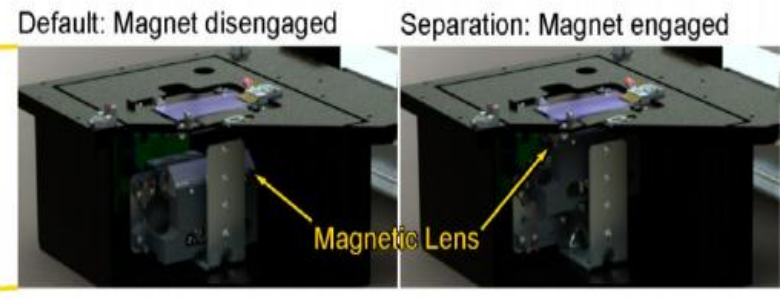

Figure 4.2 Integrated platform for DMF particle-based immunoassays. (A) Photograph of the automation setup (opened orientation) with labels showing the DMF device and Pogo pin interface. (B) Cross-sectional computer-aided design (CAD) rendering of the integrated photomultiplier (PMT) in the default (left) or measure (right) state. (C) Crosssectional CAD rendering of the motor-controlled magnet in the default (left) or separation (right) state. The automation setup is in closed orientation in (B) and (C). Reprinted (adapted) with permission from ref. 87. Copyright 2013 American Chemical Society.

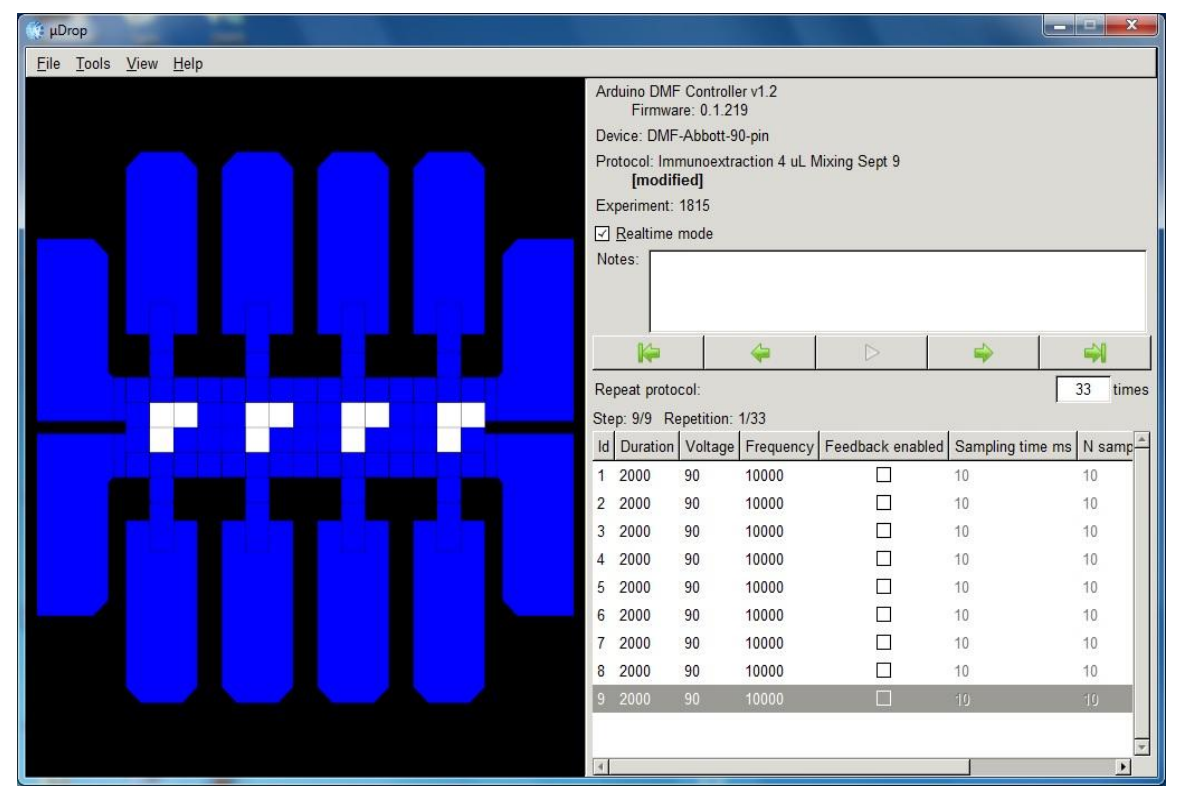

Figure 4.3 Screenshot from the custom Microdrop software demonstrating live video overlay. 
This magnet provides approximately $600 \mu \mathrm{N}$ of force which exceeds the minimal threshold for magnetic separation $(470 \mu \mathrm{N}) .{ }^{87}$ As described in $\mathrm{Ng}$ et al.'s paper ${ }^{86}$, the force on a magnetic particle inside a magnetic field is determined by the volume of the particle $(\mathrm{V})$, the magnetic susceptibility of the particle $(\chi)$, and the magnetic flux density (B). ${ }^{99}$ Assuming that the particle is in a non-magnetic medium with negligible susceptibility (e.g. air), the force on the particle is given by:

$$
F=\frac{V \cdot \chi}{\mu_{0}}(B \cdot \nabla) B
$$

where $\mu_{0}$ is the permeability of free space. The force was then obtained with the help of finite element analysis software, COMSOL (Burlington, MA; Model: 3D, Magnetic Fields, No Currents).

Droplet driving voltages were between $100-120 \mathrm{~V}_{\mathrm{RMS}}$ at $10 \mathrm{kHz}$ (sine wave). Four $4 \mu \mathrm{L}$ unit droplets were dispensed from reservoirs, merged and mixed on the device electrode array. For incubation, the droplets were moved in a modified figure ' 8 ' pattern to ensure proper dispersal of particles in a circular motion across four electrodes. During sample mixing and incubation, droplets were continuously mixed to ensure smooth movement of droplets and minimize non-specific adsorption on device surface. Waste and unused fluids were removed from devices by wicking using KimWipes (KimberlyClark, Irving, TX). 


\subsubsection{On-Chip Protein Depletion Protocol}

A general overview of the strategy behind the protocol is shown in Figure 4.1B. First, paramagnetic particle beads with appropriate functionality are moved onto the device and immobilized in order to remove the supernatant. Secondly, a sample containing proteins to be depleted is actuated on to the beads. The sample is mixed with the beads. Finally, the beads are immobilized again and the now depleted protein sample is actuated away. As shown in Figure 4.4, the actual on-chip protein depletion was performed in eight steps: (1) One droplet each of magnetic beads and protein samples (4 $\mu \mathrm{L}$ each) were loaded on the device, (2) magnetic beads were actuated into the device array, (3) the magnet was engaged to immobilize the beads onto the device surface, (4) the supernatant was removed from the beads, (5) the protein sample was merged with the beads and the beads were dispersed, (6) the suspension was actively incubated (moved in a figure- 8 pattern, as above) for $10 \mathrm{~min}$, (7) the beads were immobilized and the supernatant was separated from the beads, and (8) the depleted protein sample was collected in the reservoir for removal and subsequent analysis. In practice, the eight-step procedure was typically performed on four protein samples in parallel. 

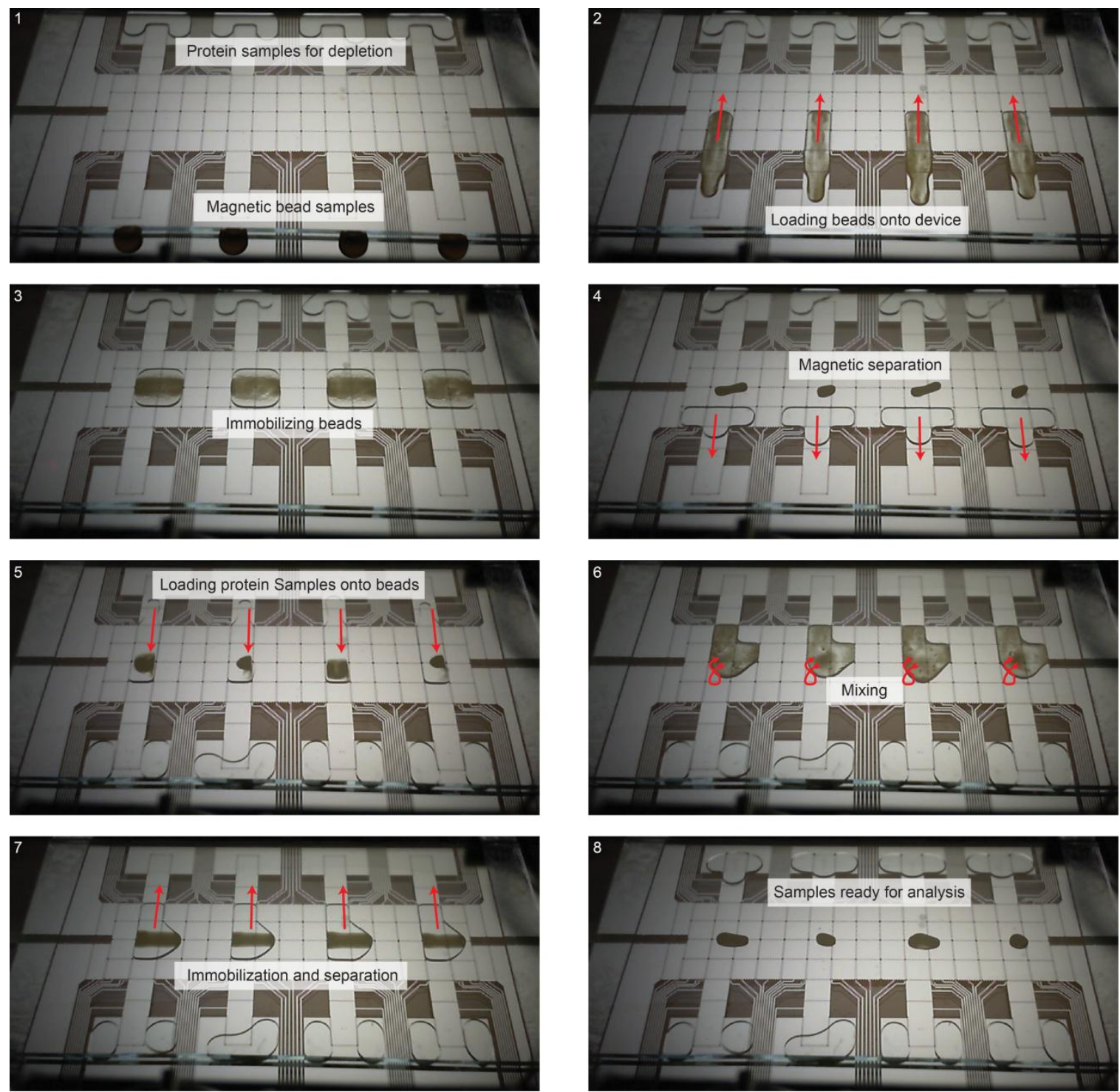

Figure 4.4 Frames from a video depicting the process of protein depletion from a sample. The dark areas on the array are the magnetic beads.

\subsubsection{Fluorescent Characterization of On-Chip Depletion}

The kinetics of on-chip depletion was probed using FITC-IgG and a suspension of protein A/G beads, using a variation of the protocol described above. Briefly, steps (1-5) were applied to a sample of FITC-IgG $(0.5 \mathrm{mg} / \mathrm{mL})$, which was then incubated for only 
$30 \mathrm{~s}$ in step (6). After steps (7-8), the supernatant droplet was driven to an unused portion of the device, and the fluorescence intensity was probed by loading the device into a plate reader (PHERAstar microplate reader, BMG Labtech, Ortenberg, Germany). Measurements were performed in "well scanning mode" (COSTAR 96 well plate geometry) with three flashes per scan point and a gain of 100 using $485 \mathrm{~nm}$ excitation, and $520 \mathrm{~nm}$ emission. After measurement, the depletion was continued by re-suspending the beads in the FITC-IgG droplet and mixing for an additional 30 seconds, followed by extracting the supernatant (steps 5-8) for another measurement of fluorescence. This procedure (deplete for 30 seconds, extract supernatant, measure fluorescence, and resuspend beads) was repeated until 10 minutes of total incubation time was completed. Fluorescence measurements were carried out on three samples with fluorescence determined for each sample following 30 seconds depletions.

The efficiency of on-chip depletion of both IgG and HSA was tested using a suspension of protein $\mathrm{A} / \mathrm{G}$ and anti-HSA beads. The two analytes were evaluated separately. For $\mathrm{IgG}$, a droplet of $0.5 \mathrm{mg} / \mathrm{mL}$ FITC-IgG was extracted (steps 1-8) with active incubation for $10 \mathrm{~min}$ in step (6). In step (8), the supernatant droplet was driven to an unused portion of the device, and the fluorescence intensity was probed as above. The supernatant droplet was then extracted again (steps 1-8) using a fresh aliquot of beads, followed by a second fluorescence intensity measurement. For HSA, an identical process was used to extract $0.5 \mathrm{mg} / \mathrm{mL}$ FITC-HSA, except with a gain of 125 in the fluorescence intensity measurements. For all fluorescence experiments, the intensity data were 
normalized to the control intensity (before depletion) to obtain relative values. Fluorescence measurements were carried out on three to four samples with fluorescence determined for each sample following 10 minute depletions. Blank measurements were taken from on-chip regions with no droplets.

\subsubsection{MALDI-MS Characterization of On-Chip Depletion}

Protein mixture solutions containing two high-abundance proteins $(2 \mathrm{mg} / \mathrm{mL}$ human $\operatorname{IgG}$ and $0.5 \mathrm{mg} / \mathrm{mL} \mathrm{HSA}$ ), and one lower-abundance protein, $0.1 \mathrm{mg} / \mathrm{mL}$ hemopexin were depleted by DMF as described above. Samples were collected for MALDI-MS analysis before depletion, after a single round of depletion with one aliquot of mixed beads, and after two rounds of depletion with two aliquots of mixed beads. Depleted samples were collected for analysis by removing the top plate and transferring the sample droplet by pipette. Four replicates were collected and evaluated for each single and double depletion experiment.

After processing by DMF, each sample was purified using a ZipTip ${ }^{\circledR} \mathrm{C} 4$ or $\mathrm{C} 18$ for tryptic digested cytochrome $\mathrm{C}$ according to the manufacturer's instructions. Briefly, ZipTip $^{\circledR} \mathrm{C} 4$ or $\mathrm{C} 18$ tips were wetted in $50 \%$ ACN containing $0.1 \%$ TFA (5x) and then equilibrated in $0.1 \%$ TFA $(5 x)$. After equilibrating, fluid in the ZipTip ${ }^{\circledR} \mathrm{C} 4$ or $\mathrm{C} 18$ pipette tips were drawn in and out of the tip for 20 cycles for maximum binding of complex mixtures, and then washed with $0.1 \%$ TFA (3x). Finally, samples were eluted directly in SA or $\alpha$-CHCA matrix solution and deposited onto a stainless steel MALDI target plate. After drying, spots were analyzed using PerSeptive Biosystems Voyager DE 
Pro MALDI-TOF Mass Spectrometer (AB Sciex, Framingham, MA, USA) operating over a mass to charge ratio (m/z) range of 20000-200000 and 700-4000 for tryptic digested cytochrome C. A total of 250 shots were collected per spectrum, with laser power fluence adjusted to optimize the signal to noise ratio $(\mathrm{S} / \mathrm{N})$. Data were processed by baseline correction, resolution (set to 100), and smoothed noise (default settings) using Voyager Data Explorer software. Signals were extracted from prominent peak heights, and root-mean square noise $\left(\mathrm{N}_{\mathrm{RMS}}\right)$ values were estimated from a spectra region with no prominent peaks (m/z 88000 -120000).

To match peaks with peptides and find sequence coverage, spectra of enzyme digests were analyzed with Mascot protein identification package searching the SwissProt database. The database was searched with one missed cleavage allowed, a mass accuracy of $50 \mathrm{ppm}$, and with a fixed carboxamidomethyl-cysteine modification and oxidized Met variable.

\subsubsection{Proteomic In-Solution Digestion and Identification of Peptides with MALDI- MS and LC-MS/MS}

A sample containing $0.5 \mathrm{mg} / \mathrm{mL}$ cytochrome $\mathrm{C}$ was mixed with $2 \mathrm{mg} / \mathrm{mL} \mathrm{IgG}$ and $0.5 \mathrm{mg} / \mathrm{mL} \mathrm{HSA}$, and the double depletion process as described above was repeated on the mixture. Tryspin solution was added to the depleted protein mixture after processing by DMF at an enzyme to substrate ratio of 1:5 (w/w). The digestion mixture was then incubated for 12 hours at $37{ }^{\circ} \mathrm{C}$ and then quenched by adding $5 \%$ formic acid. Three replicate samples were evaluated. 
Following in-solution enzymatic digestion of proteins, the tryptic peptides were first analysed using MALDI-MS and then by LC-MS/MS using an Orbitrap Velos Pro (Thermo Scientific, Bremen, Germany) mass spectrometer coupled to a nano-LC system, Easy LC, and nano-ESI source (Thermo Fischer Scientific, Bremen, Germany). Gradient Elution was employed for the LC separation where eluent A was aqueous formic acid $(0.1 \%, v / v)$ and eluent B was formic acid $(0.1 \%, v / v)$ in $\mathrm{ACN}$. The $10 \mu \mathrm{L}$ samples were injected by autosampler onto the trap column $(\mathrm{C} 18$, internal diameter $100 \mu \mathrm{m}$, length 20 $\mathrm{mm}$, particle diameter $5 \mu \mathrm{m}$ ). The peptides were then separated on an analytical column (C18, internal diameter $75 \mu \mathrm{m}$, length $100 \mathrm{~mm}$, particle diameter $5 \mu \mathrm{m}$ ) with a flow rate of $30 \mathrm{~nL} / \mathrm{min}$ and a two-step gradient (5 to $50 \%$ eluent $\mathrm{B}$ over $70 \mathrm{~min}$, followed by increasing eluent B to $100 \%$ over 45 min where it is maintained at $100 \%$ for an additional $28 \mathrm{~min}$ ). The transfer capillary temperature was set to $270{ }^{\circ} \mathrm{C}$. An ion spray voltage of 2.0 $\mathrm{kV}$ was applied to a PicoTip ${ }^{\mathrm{TM}}$ on-line nano-ESI emitter (New Objective, Berlin, Germany). Precursor ion survey scans were acquired at an Orbitrap resolution of 60,000 for $\mathrm{m} / \mathrm{z}$ range 200 to 2,000. Data were acquired using Xcalibur ${ }^{\mathrm{TM}}$ software, and processed by Sequest search engine (Proteome Discover 1.4, Thermo Fischer) against the SwissProt database, allowing up to two missed cleavage sites and a mass tolerance of $10 \mathrm{ppm}$ for precursor ion scans and $0.8 \mathrm{u}$ for product ion scans. 


\subsection{Results and Discussion}

\subsubsection{DMF Device and Method}

DMF enables the manipulation of discrete droplets on an array of electrodes and thus offers a number of advantages for sample preparation prior to biochemical analysis. We hypothesized that DMF would be a convenient platform to use for depletion of HAPs from complex proteomic samples. The device used here is shown in Figure 4.1A. Droplets of samples and reagents are loaded into reservoir electrodes, where they can be aliquoted/dispensed, mixed and separated using a defined voltage program. In addition to droplet manipulation, samples can be further manipulated using antibody functionalized paramagnetic particles. The particles can be manipulated with magnetic fields, allowing for separation of specific molecules bound to the particles from the remainder of the droplet (the "supernatant"). The interplay between magnetic forces and interfacial forces arising from droplet manipulation can be tuned by moving a magnet vertically under the device $^{87}$ (either close to the device or "engaged," or away from the device, or “disengaged").

A general scheme for HAP depletion by DMF and magnetic particle immobilization is depicted in Figure 4.1B. As shown, a droplet containing paramagnetic particles is positioned over an engaged magnet, the initial supernatant is driven away, and a second droplet containing proteomic analytes is delivered to the immobilized beads. The magnet is then disengaged allowing for resuspension of the particles and active 
mixing, followed by engaging the magnet again to allow for the particles to be separated again. The resulting supernatant droplet should (ideally) contain substantially depleted concentrations of constituents that are bound to the beads. The full process is shown in Figure 4.4, which depicts a series of images from a movie. With the device format used here, it was feasible to implement this process in a multiplexed fashion- up to four samples at a time. With the recent report of DMF devices bearing thousands of independently addressable electrodes, ${ }^{100}$ we propose that in the future, much higher levels of multiplexing might be achieved.

\subsubsection{On-chip Depletion Kinetics and Efficacy}

Two fluorescent assays were developed out to determine appropriate conditions for protein depletion. First, an assay to determine the kinetics of depletion was developed, using a fluorescently labeled variant of the HAP, IgG (FITC-IgG) and protein A/Glabeled particles (proteins A and G bind IgG with high specificity). Supernatant droplets containing FITC-IgG were probed repeatedly after successive 30 second incubations with

particles using techniques reported previously ${ }^{65,81,82}$ for on-chip fluorescence analysis. Figure 4.5A shows the trend observed for the relative fluorescence intensity and increased contact time with the protein $\mathrm{A} / \mathrm{G}$ beads. The fluorescence intensity initially decreases rapidly, but gradually stabilizes after 5 minutes of exposure to the paramagnetic particles. Following 9 minutes of exposure, the fluorescence intensity is reduced by $>95 \%$, and further depletion time did not result in a significant fluorescence intensity 
reduction. As a result, we established a conservative mixing/contact time of 10 minutes for subsequent depletion experiments.
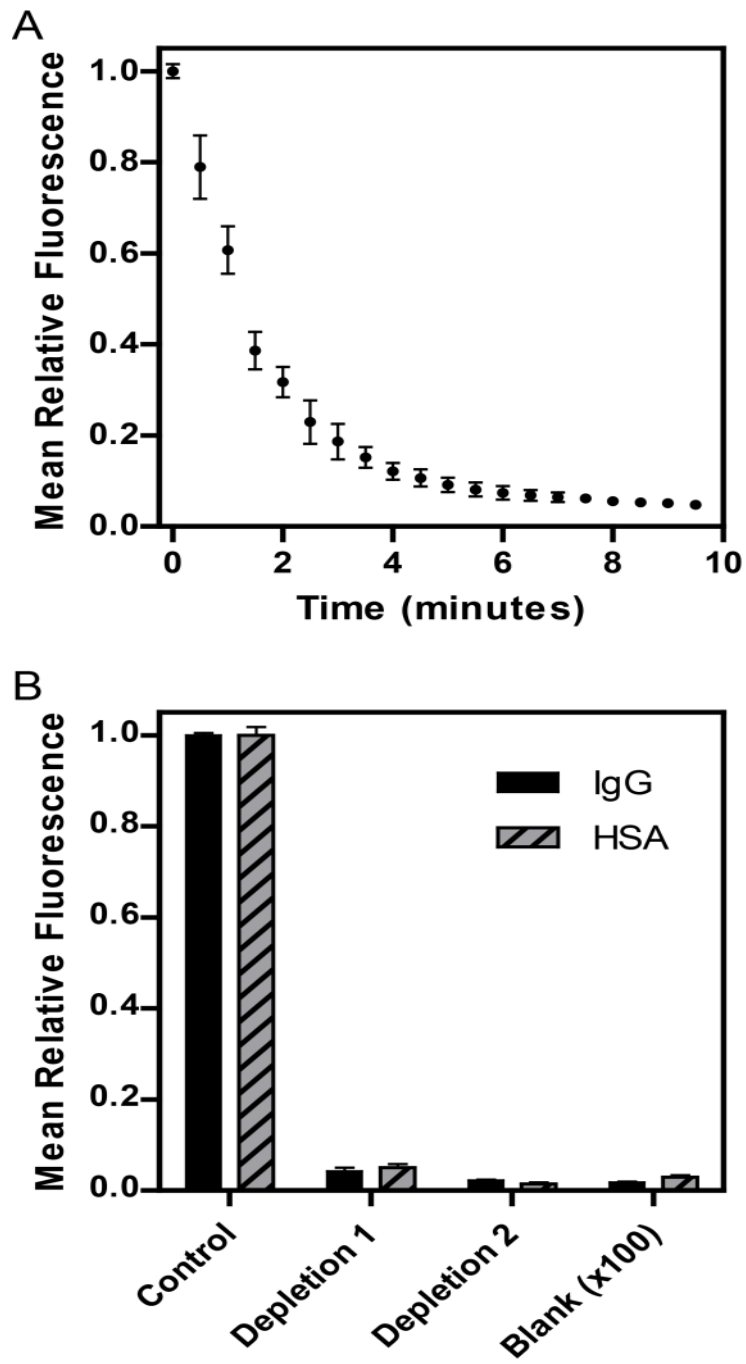

Figure 4.5 On-chip depletion kinetics and efficacy. (A) Graph of mean relative FITC-IgG fluorescence intensity (normalized to $t=0$ ) as a function of mixing time using Protein $\mathrm{A} / \mathrm{G}$ magnetic beads $(\mathrm{n}=3)$. After approximately 9 minutes the beads have depleted the IgG level by $95 \%$. Error bars represent 1 standard deviation about the mean. (B) Graph of mean relative fluorescence intensity (normalized to control) of FITC-IgG (solid, $n=4$ ) and FITC-HSA (hatched, $\mathrm{n}=3$ ) as a function of one or two 10-min depletion step(s). The magnitude of blank measurements was multiplied by 100 to illustrate the low background signal of on-chip fluorescent measurements. Error bars represent 1 standard deviation about the mean. 
The second assay was developed to evaluate the depletion efficacy for the two most prevalent HAPs in human serum: IgG (again monitored as FITC-IgG) and HSA (monitored as FITC-HSA), with a mixture of particles bearing protein A/G (for $\operatorname{IgG}$ ) and anti-HSA (for HSA). In practice (as described below), the two proteins can be depleted simultaneously, but for this assay, because the same fluorophore was used for both analytes, they were probed sequentially. As shown in Figure 4.5B, the relative fluorescence intensity decreased dramatically after a single depletion, with $>95 \%$ reduction following 10 minutes of contact. A second depletion step was then studied to explore whether the fluorescent intensity could be reduced further. A second aliquot of functionalized paramagnetic particles was actuated to the center of the chip and mixed with the sample for an additional 10 minutes. The second depletion resulted in a further fluorescence intensity reduction, $98 \%$ relative to the initial fluorescence intensities. As shown, the intensities after two 10-min depletions were similar to those observed for blank measurements, meaning that it is likely that additional depletions would not be useful. In the future, if different concentrations of proteins or densities of particles are used, it may be useful to evaluate additional (sequential) depletions. Regardless, the depletion efficiencies shown in Figure 4.5B are similar to those of other commercially available extraction methods which remove $\approx 98 \%$ of the high abundance proteins. ${ }^{101}$

\subsubsection{MALDI-MS Analysis of DMF-Based Protein Depletion}

Fluorescence measurements provide a quantitative assessment of the amount of a high abundance protein that is removed following a single and double depletion steps. 
However, little information is obtained regarding the specificity of the paramagnetic particle based depletion process. Off-chip MALDI-MS analysis was used to illustrate the specificity and detection enhancement afforded through paramagnetic particle/DMF based protein depletion. MALDI-MS has been used as a semi-quantitative profiling tool for proteomic samples. ${ }^{102}$ Note that the presence of HSA, $\operatorname{IgG}$ and hemopexin in real serum are at concentrations of $35-55 \mathrm{mg} / \mathrm{mL},{ }^{103} 6.14-12.95 \mathrm{mg} / \mathrm{mL}^{63}$ and $0.8-1.2$ $\mathrm{mg} / \mathrm{mL}^{103}$ respectively. However, because MALDI signal was dependent on the concentration of the mixture of samples used, after many trials, a mixture of HSA ( 0.5 $\mathrm{mg} / \mathrm{mL}), \operatorname{IgG}(2 \mathrm{mg} / \mathrm{mL})$ and hemopexin $(0.1 \mathrm{mg} / \mathrm{mL})$ was chosen to be used to represent a protein mixture comprised of HAPs (HSA and $\mathrm{IgG}$ ) and low-abundance proteins (hemopexin). Although this mixture is not an identical match to the concentrations of these proteins in serum, it does reflect the correct ratio of $\mathrm{IgG}$ to hemopexin (in serum, $\operatorname{IgG}$ is typically $\sim 10-20 \mathrm{x}$ more concentrated than hemopexin).

Figure 4.6 shows three representative MALDI-MS spectra for the protein mixture treated with (A) control (no depletion), (B) a single sample depletion step, and (C), two depletion steps and these represent samples highlighted in bold in Table 4.1. Four spectra were collected for each condition, and $\mathrm{N}_{\mathrm{RMS}}$ values were estimated from spectral regions without prominent peaks to determine signal-to-noise (S/N) ratios (Table 4.1). Initially, the low-abundant protein, hemopexin, produces very low relative signal intensity compared to the highly abundant species (IgG and HSA) in the protein mixture, prior to depletion Figure 4.6 (A). The HSA is the most intense signal peak at approximately 152 
$(\mathrm{S} / \mathrm{N}=92.0)$ while the singly and doubly charged intensities for the $\operatorname{IgG}$ are $22(\mathrm{~S} / \mathrm{N}=$ 23.9) and $35(\mathrm{~S} / \mathrm{N}=22.0)$ respectively. Conversely, the MALDI signal for hemopexin is quite low at approximately $13(\mathrm{~S} / \mathrm{N}=14.7)$. The low signal in the protein sample prior to extraction presumably results from charge competition/ion suppression due to presence of the HAPs. ${ }^{104}$ The mixed protein sample was depleted using the DMF bead-based protocol (vide supra) and following a single depletion step, the hemopexin MALDI signal is increased 6.7 times to 87 ( $\mathrm{S} / \mathrm{N}=38.9$ ). Conversely the HSA signal is decreased by 5.2 times to $34(\mathrm{~S} / \mathrm{N}=17.6)$ and now ranks as the fourth most intense peak behind $\mathrm{IgG}$ with intensities of $95(\mathrm{~S} / \mathrm{N}=40.9)$ and $67(\mathrm{~S} / \mathrm{N}=28.7)$, for +1 and +2 charge states respectively (Figure 4.6B). Interestingly the first depletion produces an increase in both the $\mathrm{S} / \mathrm{N}$ ratio for the $\mathrm{IgG}$ and hemopexin, resulting from reduced ion suppression from the simplified matrix.

Following a double depletion, the MALDI signals for HSA and IgG are diminished to: $5.5(\mathrm{~S} / \mathrm{N}=11.1)$ for $\mathrm{HSA}$ which corresponds to a 6.2 times reduction compared to the first depletion, and a total reduction of 27.6 compared to the original sample; and IgG where the signal is reduced to $16(\mathrm{~S} / \mathrm{N}=17.5)$ and $8.1(\mathrm{~S} / \mathrm{N}=11.1)$ for the singly and doubly charged ions, respectively, with an overall average reduction of 2.5 for both IgG ions. Conversely, the hemopexin signal is now the most intense signal at 76 $(\mathrm{S} / \mathrm{N}=62.3)$ in the MALDI-MS spectrum with a signal enhancement of 5.6 and an improvement in signal to noise ratio of 4.2. Detailed results of $\mathrm{S} / \mathrm{N}$ for each replicate are tabulated in Table 4.1 . 
A significant enhancement for hemopexin is observed when comparing the signal to noise $(\mathrm{S} / \mathrm{N})$ ratios of the peaks in the protein mixture before depletion, and following a single, and double depletion. Furthermore, the signal to noise ratios also point to the necessity of conducting a second depletion step as significant protein concentration remains after the first step to limit the signal to noise enhancement.

Table 4.1 Comparison of S/N ratios for Ion Intensities in MALDI-MS spectra for control, following a single, and double depletion, with the DMF/magnetic bead platform

\begin{tabular}{|c|c|c|c|c|}
\hline \multirow[b]{2}{*}{ S/N Ratios } & \multicolumn{4}{|c|}{ Analyte and S/N Ratio } \\
\hline & $\begin{array}{c}\text { Hemopexin } \\
(\mathrm{M}+\mathrm{H})^{+} \\
\end{array}$ & $\begin{array}{c}\text { HSA } \\
(\mathbf{M}+\mathbf{H})^{+} \\
\end{array}$ & $\begin{array}{c}\text { IgG } \\
(\mathrm{M}+2 \mathrm{H})^{2+} \\
\end{array}$ & $\operatorname{IgG}(\mathrm{M}+\mathrm{H})^{+}$ \\
\hline Control (No Depletion) & 14.65 & 92.04 & 21.98 & 23.35 \\
\hline Single Depletion 1 & 16.71 & 16.32 & 29.85 & 42.58 \\
\hline Single Depletion 2 & 38.81 & 17.61 & 28.66 & 40.90 \\
\hline Single Depletion 3 & 13.53 & 12.73 & 25.07 & 25.87 \\
\hline Single Depletion 4 & 22.21 & 15.78 & 23.81 & 28.63 \\
\hline Mean Single Depletion & 22.81 & 15.61 & 26.85 & 34.49 \\
\hline$\sigma$ Single Depletion & 11.25 & 2.07 & 2.87 & 8.47 \\
\hline Double Depletion 1 & 21.58 & 7.37 & 11.05 & 12.10 \\
\hline Double Depletion 2 & 40.72 & 10.66 & 14.54 & 17.94 \\
\hline Double Depletion 3 & 31.41 & 8.12 & 11.91 & 14.08 \\
\hline Double Depletion 4 & 62.25 & 8.14 & 11.05 & 17.45 \\
\hline Mean Double Depletion & 38.99 & 8.57 & 12.14 & 15.39 \\
\hline$\sigma$ Double Depletion & $\mathbf{1 7 . 3 7}$ & 1.44 & 1.65 & 2.78 \\
\hline
\end{tabular}




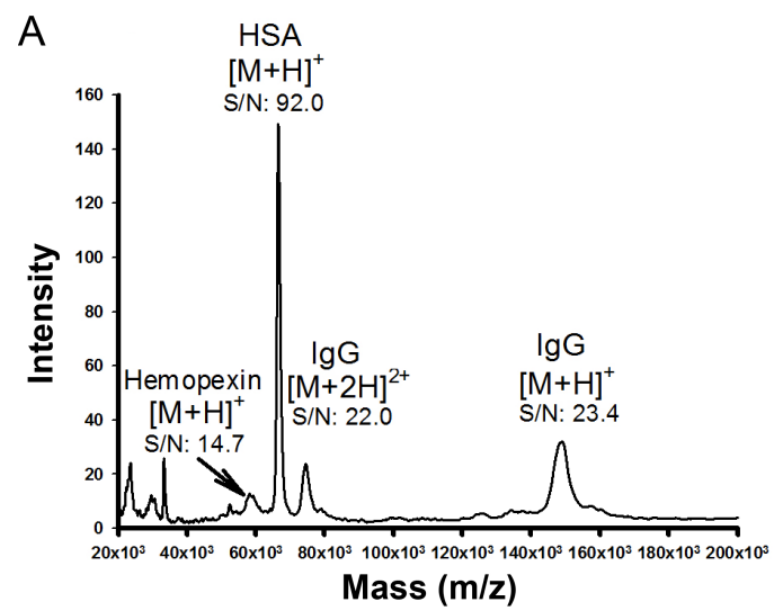

B

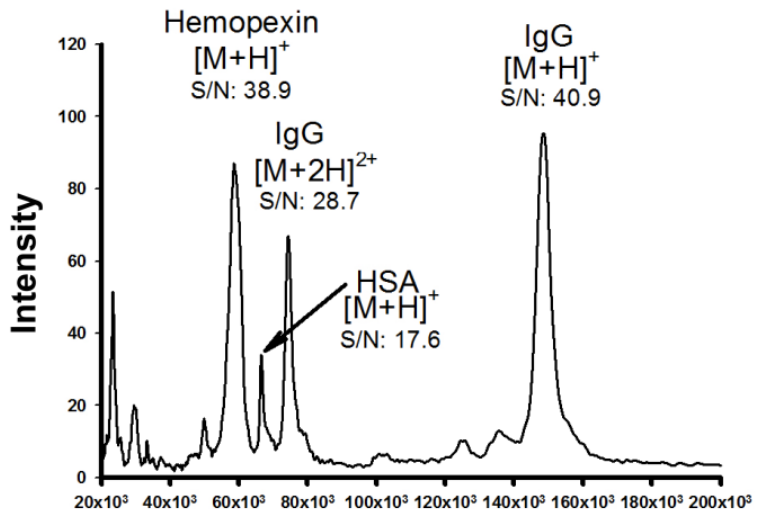

Mass $(\mathrm{m} / \mathrm{z})$

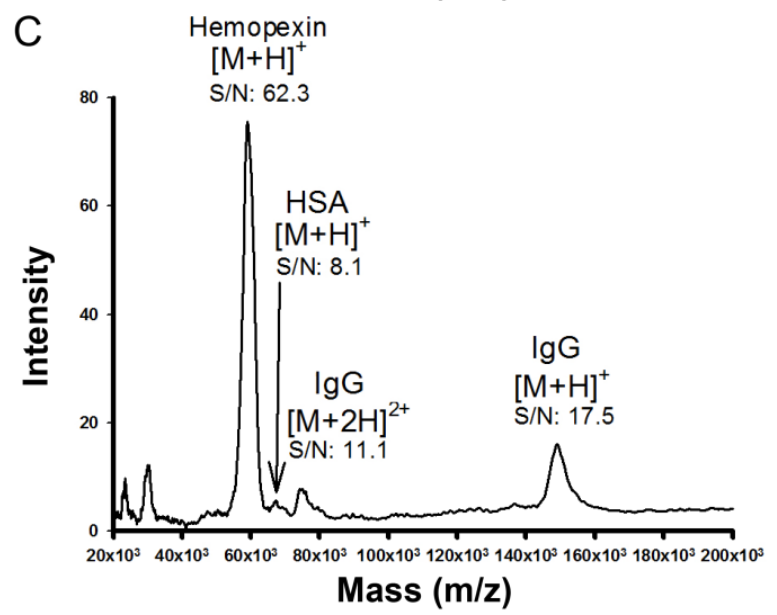

Figure 4.6 MALDI Spectra of sample comprising HSA (0.5 mg/mL), IgG $(2 \mathrm{mg} / \mathrm{mL})$ and Hemopexin $(0.1 \mathrm{mg} / \mathrm{mL})$. (A) Before depletion, (B) after single depletion, and (C) after double depletion. 


\subsubsection{Proteomic In-Solution Digestion and Identification of Peptides with MALDI- MS and LC-MS/MS}

Bottom up proteomic analysis involves the digestion of a protein sample with a proteolytic enzyme prior to mass spectrometric analysis. To examine the compatibility of the DMF-based protein depletion protocol with MS, a small model protein, cytochrome $\mathrm{C}$, was used in the protein mixture to replace hemopexin because it does not require complex denaturation steps prior to digestion. Following paramagnetic particle DMFbased depletion the cytochrome $\mathrm{C}$ was digested off-chip using trypsin, and then subjected to MALDI-MS and LC-MS/MS for the identification of tryptic peptides.

For analysis by MALDI-MS, after processing by Mascot search, we found that there are 11 peaks (peptides) matched with cytochrome $C$ horse (Figure 4.7). The score was calculated to be 225 , and considerably higher than 88 , which showed proof that the digestion result was significant. However, sequence coverage was only found to be $68 \%$. Typically, using LC MS/MS typical sequence coverage is enhanced (>80\%) due to reduced charge competition. ${ }^{105}$ 


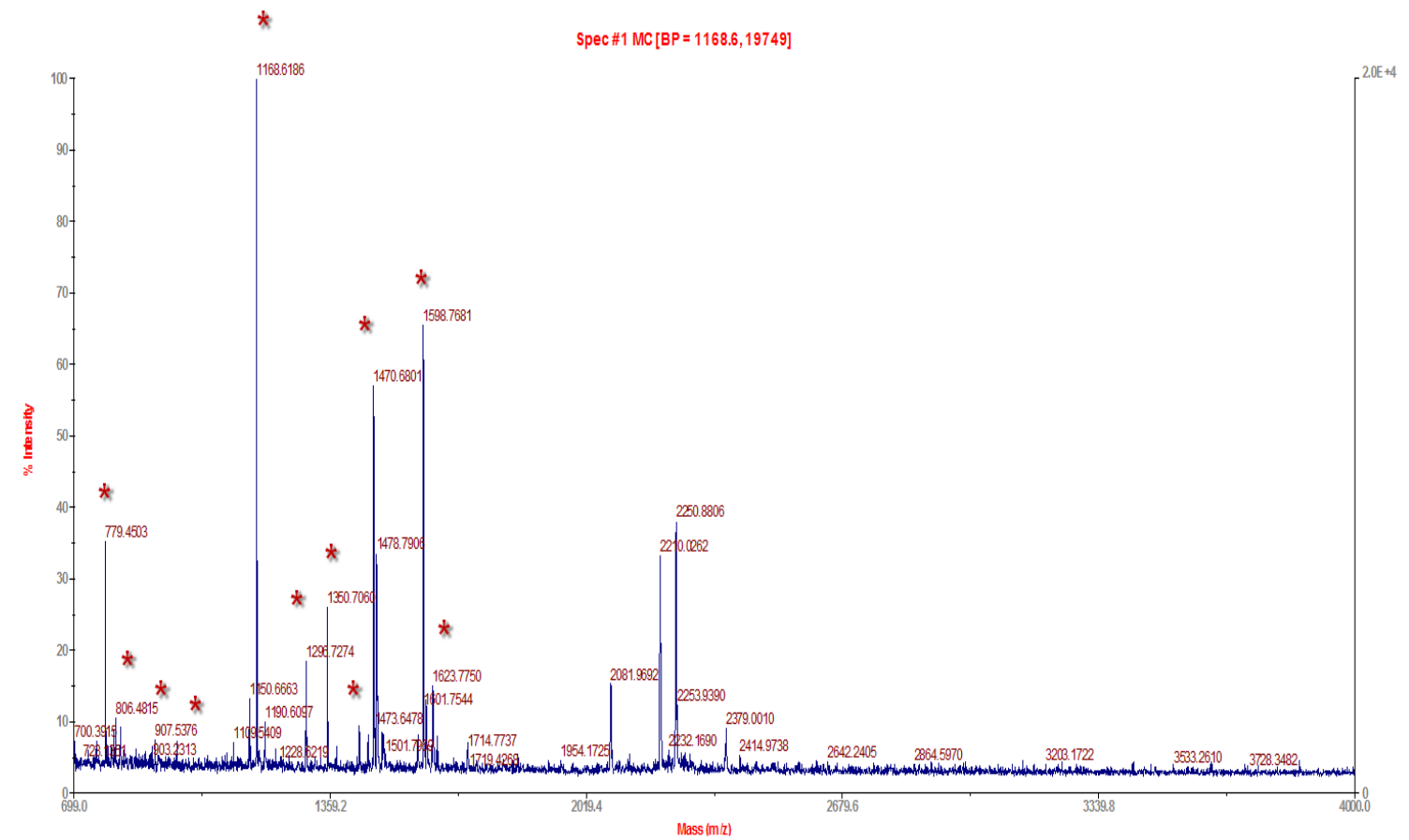

Figure 4.7 MALDI-MS spectrum for digestion of cytochrome C, 11 peptides matched, score 225 and sequence coverage of $68 \%$.

Thus, we repeated the protein depletion and digestion protocol and subjected to LC-MS/MS. After repeating the analysis three times, sequence coverage was found to be $88.6 \%$ for cytochrome $\mathrm{C}$, which suggests the entire depletion and digestion process using the magnetic bead depletion and DMF was very efficient. A table summarizing peptides found and other related parameters were listed in Table 4.2. 
Table 4.2 Sequence Peptides of Trypsin Digested Cytochrome C using LC-MS/MS

\begin{tabular}{|c|c|c|c|c|c|}
\hline A3 & Sequence & q-Value & Charge & $\mathrm{MH}^{+}[\mathrm{Da}]$ & $\begin{array}{c}\Delta \mathrm{M} \\
{[\mathrm{ppm}]}\end{array}$ \\
\hline High & KTGQAPGFSYTDANKNK & 0 & 3 & 1826.91726 & 7.69 \\
\hline High & KTGQAPGFSYTDANK & 0 & 2 & 1584.77823 & 8.15 \\
\hline High & HKTGPNLHGLFGRK & 0 & 2 & 1561.88294 & 7.60 \\
\hline High & EETLmEYLENPKK & 0 & 2 & 1639.80069 & 7.47 \\
\hline High & GITWKEETLmEYLENPK & 0 & 2 & 2097.04155 & 9.81 \\
\hline High & TGPNLHGLFGR & 0 & 2 & 1168.62871 & 5.56 \\
\hline High & KTEREDLIAYLK & 0 & 2 & 1478.82998 & 5.81 \\
\hline High & TGQAPGFSYTDANK & 0 & 2 & 1456.68292 & 8.63 \\
\hline High & TGQAPGFSYTDANKNK & 0 & 2 & 1698.82109 & 7.56 \\
\hline High & EETLmEYLENPK & 0 & 2 & 1511.70673 & 8.77 \\
\hline High & KYIPGTKmIFAGIK & 0 & 3 & 1582.91380 & 7.06 \\
\hline High & EETLMEYLENPK & 0 & 2 & 1495.71135 & 8.56 \\
\hline High & HKTGPNLHGLFGR & 0 & 3 & 1433.78747 & 7.93 \\
\hline High & TEREDLIAYLK & 0 & 2 & 1350.73733 & 8.08 \\
\hline High & MIFAGIKK & 0 & 2 & 907.55052 & 7.81 \\
\hline High & mIFAGIKK & 0 & 2 & 923.54544 & 7.68 \\
\hline High & GGKHKTGPNLHGLFGR & 0 & 4 & 1675.92532 & 6.75 \\
\hline High & mIFAGIK & 0 & 2 & 795.45102 & 9.61 \\
\hline High & YIPGTKmIFAGIK & 0 & 2 & 1454.81778 & 6.96 \\
\hline High & KYIPGTKMIFAGIK & 0.001 & 3 & 1566.91741 & 6.19 \\
\hline High & TGPNLHGLFGRK & 0.006 & 2 & 1296.72638 & 7.09 \\
\hline Medium & TEREDLIAYLKK & 0.036 & 3 & 1478.83082 & 6.39 \\
\hline Low & EDLIAYLKK & 0.1 & 2 & 1092.63980 & 8.99 \\
\hline Low & CAQCHTVEK & 0.103 & 2 & 1018.45439 & 9.71 \\
\hline Low & KYIPGTK & 0.214 & 2 & 806.48480 & 9.56 \\
\hline Low & EETLmEYLENPKKYIPGTK & 0.268 & 3 & 2299.17240 & 8.56 \\
\hline Low & IFVQK & 0.269 & 2 & 634.39791 & 8.84 \\
\hline Low & KIFVQK & 0.335 & 2 & 762.49372 & 8.45 \\
\hline Low & MIFAGIKKK & 0.349 & 3 & 1035.64629 & 7.62 \\
\hline Low & MIFAGIK & 0.414 & 2 & 779.45461 & 7.89 \\
\hline Low & EDLIAYLK & 0.426 & 2 & 964.54332 & 8.62 \\
\hline Low & YIPGTK & 0.449 & 2 & 678.38850 & 9.40 \\
\hline Low & YIPGTKMIFAGIKK & & 3 & 1566.91741 & 6.19 \\
\hline Low & mIFAGIKKK & & 3 & 1051.64138 & 7.66 \\
\hline Low & KKTER & & 2 & 661.40571 & 9.87 \\
\hline
\end{tabular}


It was very interesting to learn also that when we subjected a tryptic digested mixture of IgG, HSA and cytochrome $\mathrm{C}$ without extraction into LC-MS/MS, the same sequence coverage, $88.6 \%$, was obtained. Since the LC-MS/MS is capable of separating the complex mixture in the chromatography column before injecting the sample to the MS detector, high sequence coverage can be obtained even without depletion. Although we expected to get this result from our LC-MS/MS, this result actually counteracted the significance of our research project unless we could prove that both depletion and digestion process could be done on-chip, and this would help us eliminating long sample processing time that we would have to deal with current commercially available depletion methods for depletion of a single protein on columns such as the ProtoPrep ${ }^{\circledR} 20$ (SigmaAldrich), ${ }^{106}$ which require between $20-60$ minutes for completion for a single sample. Since we have proved that we could successfully complete the depletion process within 10 minutes for multiple samples simultaneously (up to four on the current device), our research is still significant in that it eliminates the need for lengthy depletion protocols, high levels of sample dilution or both. 


\section{Chapter 5 - Conclusions and Future Work}

\subsection{Conclusions}

The state of the art in DMF technology describing the theory of droplet actuation, device fabrication and integration, and applications are summarized in the introduction. Of those applications discussed, proteomics and clinical diagnostics seem to be the most attractive targets for DMF, in which complex samples can be pretreated and analyzed on a single device.

This thesis presents the use of DMF for separation of high-abundance and lowabundance proteins with antibody-immobilized magnetic beads for a human plasma protein depletion application due to its advantages of reduced reagent consumption and dilution, and faster analysis. DMF is performed with an automated controller system and the protein depletion process was monitored both on-chip (fluorescence) manually and off-chip with MALDI-MS.

Initially, DMF PCB and glass devices were fabricated in a clean room and tested manually with controller system. After testing the DMF devices on two different substrate materials in a one-plate and two-plate configuration, we found PCB devices did not work properly due to fabrication problems and only the two-plate configuration enabled droplet movement with glass devices. The limited success of initial tests was believed caused by fabrication problems, limited chip robustness or contamination of the 
Teflon $^{\circledR}$-AF surface. 12 new devices were prepared (under the guidance of Dr. Wheeler's students at the University of Toronto where five of them were SU-8 coated with the remainder of the chips coated with Parylene-C as the dielectric layer. After testing each device, we realized that devices with Parylene-C were defective since the primer used to adhere parylene to the electrode patterned substrate was missed during the fabrication process. This led to the Parylene-C dielectric layer being peeled off after limited use. Alternatively, SU-8 coated substrates were successfully demonstrated as working devices by applying AC potentials $\left(\sim 150 \mathrm{~V}_{\mathrm{RMS}}, 18 \mathrm{kHz}\right)$ with a manual voltage controller. These devices were used for initial protein depletion experiments employing immunochemistry and magnetic bead-based pull-down techniques where the complete protein depletion process using model reagents and beads was successfully demonstrated. The efficiency of IgG protein depletion using magnetic beads coated with Protein $A / G$, on the DMF platform was carried out off-line, and the presence of $\operatorname{IgG}$ before and after depletions detected with MALDI-MS. Results showed that Pluronic F127 (present to inhibit proteins adsorbing to the hydrophobic layer of the DMF substrate) decreased the binding of IgG to the magnetic beads. Switching to a different surfactant (Pluronics F68) largely solved this problem since it did not adversely affect the antibody antigen interaction, while preventing non specific protein adsorption.

Finally, we have demonstrated that using our new method, protein depletion was successfully employed on DMF using an automated system in the Wheeler laboratory. The depletion process is shown to be a powerful tool for rapid, efficient and automated 
sample processing by achieving $98 \%$ depletion efficiency in as little as 10 minutes for multiple samples simultaneously (up to four on the current device). This results in an approximately 3-fold increase in S/N ratio in MALDI-MS analysis for a low abundant protein, hemopexin. We demonstrate that the depletion process is sufficient for tryptic digest analysis as well as capable of $89 \%$ sequence coverage for cytochrome $\mathrm{C}$ from a depleted sample.

Compared to current commercially available depletion methods for depletion of a single protein on columns such as the ProtoPrep ${ }^{\circledR} 20$ (Sigma-Aldrich) $^{106}$ which require between 20 - 60 minutes for completion for a single sample, the new DMF based method eliminates the need for lengthy depletion protocols and high levels of sample dilution. Furthermore we believe the new technique has great potential for biomarker identification at near-patient/point of care settings worldwide.

\subsection{Future Work}

As described previously, although depletion can be carried out on DMF in 10 minutes, the LC-MS/MS follow-up analysis was still required for identification of tryptic peptides. It would be very useful if we could carry out both depletion and digestion onchip and then introduce to LC-MS/MS for peptides identification. This will help to

eliminate the significant time required for the depletion process as well as severe dilutions of unbound protein fractions and sample loss during subsequent preconcentration step using LC-columns based methods. 
Moreover, LC-MS/MS was able to separate a mixture of IgG, HSA and cytochrome $\mathrm{C}$ because there were only three proteins in the mixture. Thus, it would be helpful if we could use a mixture of many different proteins (e.g. human plasma sample). Since it may be very difficult to employ real plasma samples on DMF due to protein adsorption problems even with the addition of Pluronic, we suggest repeating the depletion process with simulated human plasma on DMF, and if we can prove that this method works with the human plasma, then it would be more convincing to prove that our new method has considerable advantages compared to LC-MS/MS for tryptic peptide analysis. 


\section{REFERENCES}

(1) Jebrail, M. J.; Bartsch, M. S.; Patel, K. D. Lab on a Chip 2012, 12, 2452.

(2) Wheeler, A. R. Science 2008, 322, 539.

(3) Teh, S. Y.; Lin, R.; Hung, L. H.; Lee, A. P. Lab on a Chip 2008, 8, 198.

(4) Tabeling, P. Lab on a Chip 2009, 9, 2428.

(5) Jebrail, M. J.; Wheeler, A. R. Analytical Chemistry 2009, 81, 330.

(6) Mousa, N. A.; Jebrail, M. J.; Yang, H.; Abdelgawad, M.; Metalnikov, P.; Chen, J.; Wheeler, A. R.; Casper, R. F. Science Translational Medicine 2009, 1, 1 ra2.

(7) Pollack, M. G.; Fair, R. B.; Shenderov, A. D. Applied Physics Letters 2000, 77, 1725.

(8) Lee, J.; Moon, H.; Fowler, J.; Schoellhammer, T.; Kim, C. J. Sensors and Actuators a-Physical 2002, 95, 259.

(9) Mitchell, L. Digital chip analyzes blood from tiny drop Futurity [Online Early Access]. Published Online: 2011. http://www.futurity.org/digital-chip-analyzes-blood-from-tinydrop/ (accessed August, 2011).

(10) Jebrail, M. J.; Yang, H.; Mudrik, J. M.; Lafreniere, N. M.; McRoberts, C.; AlDirbashi, O. Y.; Fisher, L.; Chakraborty, P.; Wheeler, A. R. Lab on a Chip 2011, 11, 3218.

8,672 .

(11) Abdelgawad, M.; Freire, S. L. S.; Yang, H.; Wheeler, A. R. Lab on a Chip 2008,

(12) Abdelgawad, M.; Wheeler, A. R. Advanced Materials 2009, 21, 920.

(13) Cooney, C. G.; Chen, C. Y.; Emerling, M. R.; Nadim, A.; Sterling, J. D. Microfluidics and Nanofluidics 2006, 2, 435.

(14) Malic, L.; Brassard, D.; Veres, T.; Tabrizian, M. Lab on a Chip 2010, 10, 418.

(15) Barbulovic-Nad, I.; Au, S. H.; Wheeler, A. R. Lab on a Chip 2010, 10, 1536.

(16) Cho, S. K.; Moon, H. J.; Kim, C. J. Journal of Microelectromechanical Systems 2003, 12,70 .

(17) Srinivasan, V.; Pamula, V. K.; Fair, R. B. Lab on a Chip 2004, 4, 310. 
(18) Moon, H.; Wheeler, A. R.; Garrell, R. L.; Loo, J. A.; Kim, C. J. Lab on a Chip 2006, 6, 1213.

(19) Berthier, J. Microdrops and Digital Microfluidics; William Andrew Pub.: Norwich, NY, 2008.

(20) Baird, E.; Young, P.; Mohseni, K. Microfluidics and Nanofluidics 2007, 3, 635.

(21) Chatterjee, D.; Shepherd, H.; Garrell, R. L. Lab on a Chip 2009, 9, 1219.

(22) Fair, R. Microfluidics and Nanofluidics 2007, 3, 245.

(23) Chatterjee, D.; Hetayothin, B.; Wheeler, A. R.; King, D. J.; Garrell, R. L. Lab on a Chip 2006, 6, 199.

(24) Abdelgawad, M.; Wheeler, A. R. Advanced Materials 2007, 19, 133.

(25) Dhindsa, M.; Kuiper, S.; Heikenfeld, J. Thin Solid Films 2011, 519, 3346.

(26) Nelson, W. C.; Kim, C.-J. C. Journal of Adhesion Science and Technology 2012, $26,1747$.

(27) Watson, M. W. L.; Abdelgawad, M.; Ye, G.; Yonson, N.; Trottier, J.; Wheeler, A. R. Analytical Chemistry 2006, 78, 7877.

(28) Abdelgawad, M.; Wheeler, A. R. Microfluidics and Nanofluidics 2008, 4, 349.

(29) Choi, K.; Ng, A. H.; Fobel, R.; Wheeler, A. R. Annual Review of Analytical Chemistry 2012, 5, 413.

(30) Berthier, J.; Dubois, P.; Clementz, P.; Claustre, P.; Peponnet, C.; Fouillet, Y. Sensors and Actuators a-Physical 2007, 134, 471.

(31) Hsieh, T. H.; Fan, S. K.; Ieee In Mems 2008: 21st Ieee International Conference on Micro Electro Mechanical Systems, Technical Digest 2008, p 641.

(32) Jones, T. B. Langmuir 2002, 18, 4437.

(33) Kang, K. H. Langmuir 2002, 18, 10318.

(34) Abdelgawad, M.; Park, P.; Wheeler, A. R. Journal of Applied Physics 2009, 105.

(35) Nagiel, I. F., R. AC Electrowetting Actuation of Droplets on a Digital Microfluidic Platform, 2007.

(36) Wijethunga, P. A. L.; Nanayakkara, Y. S.; Kunchala, P.; Armstrong, D. W.; Moon, H. Analytical chemistry 2011, 83, 1658. 
(37) Sista, R.; Hua, Z.; Thwar, P.; Sudarsan, A.; Srinivasan, V.; Eckhardt, A.; Pollack, M.; Pamula, V. Lab on a Chip 2008, 8, 2091.

(38) Luan, L.; Evans, R. D.; Jokerst, N. M.; Fair, R. B. Ieee Sensors Journal 2008, 8, 628.

(39) Poulos, J. L.; Nelson, W. C.; Jeon, T.-J.; Kim, C.-J. C. J.; Schmidt, J. J. Applied Physics Letters 2009, 95.

(40) Freire, S. L. S.; Wheeler, A. R. Lab on a Chip 2006, 6, 1415.

(41) Hong, J. W.; Quake, S. R. Nature Biotechnology 2003, 21, 1179.

(42) Xie, J.; Miao, Y. N.; Shih, J.; He, Q.; Liu, J.; Tai, Y. C.; Lee, T. D. Analytical Chemistry 2004, 76, 3756. 6947.

(43) Xie, J.; Miao, Y. N.; Shih, J.; Tai, Y. C.; Lee, T. D. Analytical Chemistry 2005, 77,

(44) Taniguchi, T.; Torii, T.; Higuchi, T. Lab on a Chip 2002, 2, 19.

(45) Miller, E. M.; Wheeler, A. R. Analytical Chemistry 2008, 80, 1614.

(46) Luk, V. N.; Fiddes, L. K.; Luk, V. M.; Kumacheva, E.; Wheeler, A. R. Proteomics 2012, 12, 1310.

(47) Fiddes, L. K.; Luk, V. N.; Au, S. H.; Ng, A. H. C.; Luk, V.; Kumacheva, E.; Wheeler, A. R. Biomicrofluidics 2012, 6 .

(48) Sista, R. S.; Eckhardt, A. E.; Srinivasan, V.; Pollack, M. G.; Palanki, S.; Pamula, V. K. Lab on a Chip 2008, 8, 2188.

(49) Luk, V. N.; Wheeler, A. R. Analytical Chemistry 2009, 81, 4524.

(50) Yang, H.; Luk, V. N.; Abeigawad, M.; Barbulovic-Nad, I.; Wheeler, A. R. Analytical Chemistry 2009, 81, 1061.

(51) Srinivasan, V.; Pamula, V. K.; Fair, R. B. Analytica Chimica Acta 2004, 507, 145.

(52) Jebrail, M. J.; Ng, A. H. C.; Rai, V.; Hili, R.; Yudin, A. K.; Wheeler, A. R. Angewandte Chemie-International Edition 2010, 49, 8625.

(53) Chatterjee, D.; Ytterberg, A. J.; Son, S. U.; Loo, J. A.; Garrell, R. L. Analytical chemistry 2010, 82, 2095.

(54) Wheeler, A. R.; Moon, H.; Bird, C. A.; Loo, R. R. O.; Kim, C. J.; Loo, J. A.; Garrell, R. L. Analytical Chemistry 2005, 77, 534. 
(55) Wheeler, A. R.; Moon, H.; Kim, C. J.; Loo, J. A.; Garrell, R. L. Analytical Chemistry 2004, 76, 4833.

(56) Nelson, W. C.; Peng, I.; Lee, G.-A.; Loo, J. A.; Garrell, R. L.; Kim, C.-J. C. J. Analytical Chemistry 2010, 82, 9932.

(57) Jebrail, M. J.; Luk, V. N.; Shih, S. C.; Fobel, R.; Ng, A. H.; Yang, H.; Freire, S. L.; Wheeler, A. R. Journal of visualized experiments: JoVE 2009.

(58) Barbulovic-Nad, I.; Yang, H.; Park, P. S.; Wheeler, A. R. Lab on a Chip 2008, 8, 519.

(59) Rastogi, V.; Velev, O. D. Biomicrofluidics 2007, 1.

(60) Miller, E. M.; Ng, A. H. C.; Uddayasankar, U.; Wheeler, A. R. Anal Bioanal Chem 2011, 399, 337.

(61) Hanash, S. M.; Pitteri, S. J.; Faca, V. M. Nature 2008, 452, 571.

(62) Anderson, N. L.; Anderson, N. G. Molecular \& Cellular Proteomics 2002, 1, 845.

(63) Kratz, A.; Ferraro, M.; Sluss, P. M.; Lewandrowski, K. B. New England Journal of Medicine 2004, 351, 1548.

(64) Mayne, J.; Starr, A. E.; Ning, Z.; Chen, R.; Chiang, C.-K.; Figeys, D. Analytical chemistry 2013, 86, 176.

(65) Cao, Z.; Yende, S.; Kellum, J. A.; Robinson, R. A. S. International Journal of Proteomics 2013, 2013, 8.

(66) Zeng, Z.; Hincapie, M.; Pitteri, S. J.; Hanash, S.; Schalkwijk, J.; Hogan, J. M.; Wang, H.; Hancock, W. S. Analytical chemistry 2011, 83, 4845.

(67) Stempfer, R.; Kubicek, M.; Lang, I. M.; Christa, N.; Gerner, C. ELECTROPHORESIS 2008, 29, 4316.

(68) Quero, C.; Colomé, N.; Prieto, M. R.; Carrascal, M.; Posada, M.; Gelpí, E.; Abian, J. Proteomics 2004, 4, 303.

(69) Polaskova, V.; Kapur, A.; Khan, A.; Molloy, M. P.; Baker, M. S. ELECTROPHORESIS 2010, 31, 471.

(70) Millioni, R.; Tolin, S.; Puricelli, L.; Sbrignadello, S.; Fadini, G. P.; Tessari, P.; Arrigoni, G. PloS one 2011, 6, e19603.

(71) Govorukhina, N. I.; Keizer-Gunnink, A.; van der Zee, A. G. J.; de Jong, S.; de Bruijn, H. W. A.; Bischoff, R. Journal of Chromatography A 2003, 1009, 171. 
(72) Desrosiers, R.; Beaulieu, É.; Buchanan, M.; Béliveau, R. Cell biochemistry and biophysics 2007, 49, 182.

(73) Ahmed, N.; Barker, G.; Oliva, K.; Garfin, D.; Talmadge, K.; Georgiou, H.; Quinn, M.; Rice, G. Proteomics 2003, 3, 1980.

(74) Chromy, B. A.; Gonzales, A. D.; Perkins, J.; Choi, M. W.; Corzett, M. H.; Chang, B. C.; Corzett, C. H.; McCutchen-Maloney, S. L. Journal of Proteome Research 2004, 3, 1120.

(75) Guzman, N. A.; Blanc, T.; Phillips, T. M. ELECTROPHORESIS 2008, 29, 3259.

(76) Friedrich, D.; Please, C. P.; Melvin, T. Journal of Chromatography B 2012, 910, 163.

(77) Malmstadt, N.; Yager, P.; Hoffman, A. S.; Stayton, P. S. Analytical chemistry 2003, 75, 2943.

(78) McKenzie, K. G.; Lafleur, L. K.; Lutz, B. R.; Yager, P. Lab on a Chip 2009, 9, 3543.

(79) Peoples, M. C.; Karnes, H. T. Journal of Chromatography B: Analytical Technologies in the Biomedical and Life Sciences 2008, 866, 14.

(80) Ng, A. C.; Uddayasankar, U.; Wheeler, A. Anal Bioanal Chem 2010, 397, 991.

(81) Shuford, C. M.; Hawkridge, A. M.; Burnett, J. C.; Muddiman, D. C. Analytical chemistry 2010, 82, 10179.

(82) Corrigan, L.; Jefferies, C.; Clive Lee, T.; Daly, J. Proteomics 2011, 11, 3415.

(83) Jebrail, M. J.; Wheeler, A. R. Analytical chemistry 2008, 81, 330.

(84) Yang, H.; Mudrik, J. M.; Jebrail, M. J.; Wheeler, A. R. Analytical chemistry 2011, $83,3824$.

(85) Mudrik, J. M. D., M.D.M.; Lafrenière, N.M.; Wheeler, A.R. Canadian Journal of Chemistry 2014, 92, 179.

(86) Ng, A. H. C.; Choi, K.; Luoma, R. P.; Robinson, J. M.; Wheeler, A. R. Analytical chemistry 2012, 84, 8805 .

(87) Choi, K.; Ng, A. H. C.; Fobel, R.; Chang-Yen, D. A.; Yarnell, L. E.; Pearson, E. L.; Oleksak, C. M.; Fischer, A. T.; Luoma, R. P.; Robinson, J. M.; Audet, J.; Wheeler, A. R. Analytical chemistry 2013, 85, 9638.

(88) Fobel, R.; Kirby, A. E.; Ng, A. H. C.; Farnood, R. R.; Wheeler, A. R. Advanced Materials 2014, n/a. 
(89) Shamsi, M. H.; Choi, K.; Ng, A. H. C.; Wheeler, A. R. Lab on a Chip 2014, 14, 547.

(90) Daley, A. B.; Xu, Z.; Oleschuk, R. D. Analytical chemistry 2011, 83, 1688.

(91) Burgoyne, F. A two-for-one dielectric and hydrophobic layer for digital microfluidics. [Online Early Access]. Published Online: 2011. http://blogs.rsc.org/chipsandtips/2010/07/06/a-two-for-one-dielectric-and-hydrophobic-layer-fordigital-microfluidics/ (accessed January 21st, 2014).

(92) DuPont ${ }^{\mathrm{TM}}$ Pyralux ${ }^{\circledR}$ AP flexible circuit materials Technical Data Sheet. [Online Early Access]. Published Online: 2012. http://www.dupont.com/content/dam/assets/products-andservices/electronic-electrical-materials/assets/PyraluxAPclad_DataSheet.pdf (accessed January 21st, 2014).

(93) SU-8 3000 Permanent Epoxy Negative Photoresist. [Online Early Access]. http://microchem.com/pdf/SU-8\%203000\%20Data\%20Sheet.pdf (accessed January 21st, 2014).

(94) Magnasense. Superparamagnetic Nanospheres. Technology for Diagnostics at Point of Care [Online Early Access]. http://magnasense.com/index.php?page=superparamagneticnanospheres.

(95) Long, Z.; Shetty, A. M.; Solomon, M. J.; Larson, R. G. Lab on a Chip 2009, 9, 1567.

(96) Lesur, A.; Varesio, E.; Hopfgartner, G. Journal of Chromatography A 2010, 1217, 57.

(97) Petritis, B. O.; Qian, W.-J.; Camp, D. G.; Smith, R. D. Journal of Proteome Research 2009, 8, 2157.

(98) Fobel, R.; Fobel, C.; Wheeler, A. R. Applied Physics Letters 2013, 102, 193513.

(99) Pamme, N. Lab on a Chip 2006, 6, 24.

(100) Hadwen, B.; Broder, G. R.; Morganti, D.; Jacobs, A.; Brown, C.; Hector, J. R.; Kubota, Y.; Morgan, H. Lab on a Chip 2012, 12, 3305.

(101) Zolotarjova, N.; Martosella, J.; Nicol, G.; Bailey, J.; Boyes, B. E.; Barrett, W. C. Proteomics 2005, 5, 3304.

(102) Wong, M. Y. M.; Yu, K. O. Y.; Poon, T. C. W.; Ang, I. L.; Law, M.-K.; Chan, K. Y. W.; Ng, E. W. Y.; Ngai, S.-M.; Sung, J. J. Y.; Chan, H. L. Y. ELECTROPHORESIS 2010, 31, 1721.

(103) Tolosano, E.; Altruda, F. DNA and cell biology 2002, 21, 297.

(104) Sjödahl, J.; Kempka, M.; Hermansson, K.; Thorsén, A.; Roeraade, J. Analytical chemistry 2005, 77, 827. 
(105) Hustoft, H. K.; Brandtzaeg, O. K.; Rogeberg, M.; Misaghian, D.; Torsetnes, S. B.; Greibrokk, T.; Reubsaet, L.; Wilson, S. R.; Lundanes, E. Scientific Reports 2013, 3.

(106) Schuchard, M.; Melm, C.; Crawford, A.; Chapman, H.; Wildsmith, J.; Ray, K.; Chen, D.; Scott, G. Comparison of Precipitation Methods Following Depletion of Twenty High Abundance Proteins from Human Plasma. [Online Early Access]. Published Online: 2006. http://www.sigmaaldrich.com/content/dam/sigmaaldrich/docs/Sigma/General_Information/compar_depletion_meth.pdf (accessed Jan. 17, 2013). 